\title{
PLASTIC BUCKLING OF INITIALLY IMPERFECT STIFFENED \\ CYLINDERS IN AXIAL COMPRESSION
}

by

G. A. DUFFETT

A thesis submitted in partial fulfillment of the requirements for the degree of Master of Science in the Faculty of Engineering University of Cape Town

Department of Civil Engineering

UNIVERSITY OF CAPE TOWN

September, 1981. 
The copyright of this thesis vests in the author. No quotation from it or information derived from it is to be published without full acknowledgement of the source. The thesis is to be used for private study or noncommercial research purposes only.

Published by the University of Cape Town (UCT) in terms of the non-exclusive license granted to UCT by the author. 


\section{DECLARATION}

I, Gino Alan Duffett, hereby declare that this thesis is my own work and that no part of it has been submitted for a degree at any other university.

Signed by candidate

september, 1981 


\section{DEDICATION}

I would like to dedicate this thesis to my parents and friends who have encouraged and endured me throughout this work 


\section{ACKNOWLEDGEMENTS}

I would like to extend my appreciation to Dr. B.D. Reddy*, under whose supervision this work was conducted, for the enthusiasm, assistance and constructive guidance he has given me throughout this work.

My colleagues, especially Mr. D.L. Hawla, with whom I have had many helpful discussions concerning my research.

The Council for Scientific and Industrial Research and Ove Arup and Partners for their financial assistance.

Mrs. L. Edwards for her superb handling of the complicated typing of this document.

Mr. H. Cable for the printing of the thesis.

*Lecturer, Departments of Applied Mathematics and Civil Engineering, University of Cape Town. 


\section{ABSTRACT}

Bifurcation in the plastic range of axially compressed stringerstiffened cylinders is investigated. The shell under consideration is assumed to have an initial imperfection in the form of a sinusoidal deviation both axially and circumferentially.

The constitutive relation employed here is $\mathrm{J}_{2}$ deformation theory of plasticity. This relation, as well as kinematic assumptions regarding the behaviour of the panels and stiffeners which constitute the stiffened shell, is used in the principle of virtual work to obtain a set of non-linear algebraic equations whose solution provides complete information about the prebuckling equilibrium path.

Bifurcation from the primary path is examined by making use of a functional whose first variation is zero when. two solutions to the problem are possible. This leads to an eigenvalue problem, the eigenvalue being the critical compressive load and the eigenfunction being the corresponding buckling mode.

Results are presented for shells of different geometries and material properties, a limited comparison of theoretical results as obtained by this analysis with available experimental data is also made. 


\section{NOTATION}

$a_{j} \quad$ Gaussian quadrature spacing coefficients.

A midsurface area of a panel or stiffener.

A e end cross-sectional area of a panel or stiffener.

$b_{\alpha \beta} \quad$ curvature tensor for undeformed midsurface of a panel.

$\overline{\mathrm{C}}$

ijk $\ell$ constitutive coefficients for three dimensional stress-strain law.

$C_{\alpha \beta u \gamma}$ constitutive coefficients for plane stress stress-strain law.

C constitutive matrix for plane stress.

d half-depth of stiffener.

$d_{n} \quad$ value of determinant at $n^{\text {th }}$ iteration in Illinois algorithm.

D coefficient matrix to obtain stress resultants from actual stresses for panels or stiffeners.

e eccentricity of centroid of stiffener from panel midsurface.

$\underline{\mathrm{e}}_{\mathrm{i}} \quad$ unit base vectors for panel.

E Young's modulus.

E $_{\text {S }}$ Secant modulus.

$\mathrm{E}_{\mathrm{T}} \quad$ Tangent modulus (Young's modulus if in elastic range).

$E_{\alpha \beta}$ midsurface membrane strain tensor for stiffeners.

F bifurcation functional.

F external virtual work vector.

$\underline{g}_{i} \quad$ unit base vectors for stiffeners.

$\mathrm{H}_{j} \quad$ Gaussian quadrature weight coefficients.

H functional for differences between paths of equilibrium beyond bifurcation point.

$H_{\alpha \beta} \quad$ strain tensor for stiffeners.

H strain vector for stiffeners.

I number of iterations to obtain the solution for a particular load step.

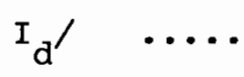


$I_{d}$ number of desired iterations to obtain the solution for a particular load step.

$\mathrm{J}_{2} \quad$ second invariant of stress deviator tensor.

J matrix of coefficients to obtain internal virtual work from stress resultants for panels or stiffeners.

$K_{\alpha \beta} \quad$ midsurface bending strain tensor for stiffeners.

$\ell \quad$ length of cylinder.

$\overline{\mathrm{L}}_{i j k \ell}$

instantaneous moduli for rate constitutive relation for three

$\mathrm{L}_{\alpha \beta K \gamma}$ instantaneous moduli for rate constitutive relation for plane stress. $m$ number of half-waves in axial direction for primary path.

$M_{\alpha \beta}, M_{\alpha \beta}$ bending moment resultants for panels and stiffeners respectively. M deformation matrix for bifurcation mode.

n strain hardening exponent.

N number of stiffeners.

$\mathrm{n}_{\alpha \beta}, \mathrm{N}_{\alpha \beta}$ membrane stress resultants for panels and stiffeners respectively. $\mathrm{p}$ number of half-waves between adjacent stiffeners in circumferential direction for bifurcation mode.

P rate constitutive matrix for panels.

q number of half-waves in axial direction for bifurcation mode; this describes the bifurcation mode.

$Q_{j n} \quad$ stiffness matrix coefficients for solution iteration procedure.

Q matrix of coefficients to obtain bifurcation functional contributions from membrane stresses for panels and stiffeners.

$r \quad$ radius of cỳlinder.

$\underline{r}, \underline{R}$ vector containing stress resultants for panels and stiffeners respectively.

$\underline{R}$ matrix of coefficients to obtain bifurcation functional contributions from differences in stress resultants for panels and stiffeners. modified Aitken acceleration factor. 


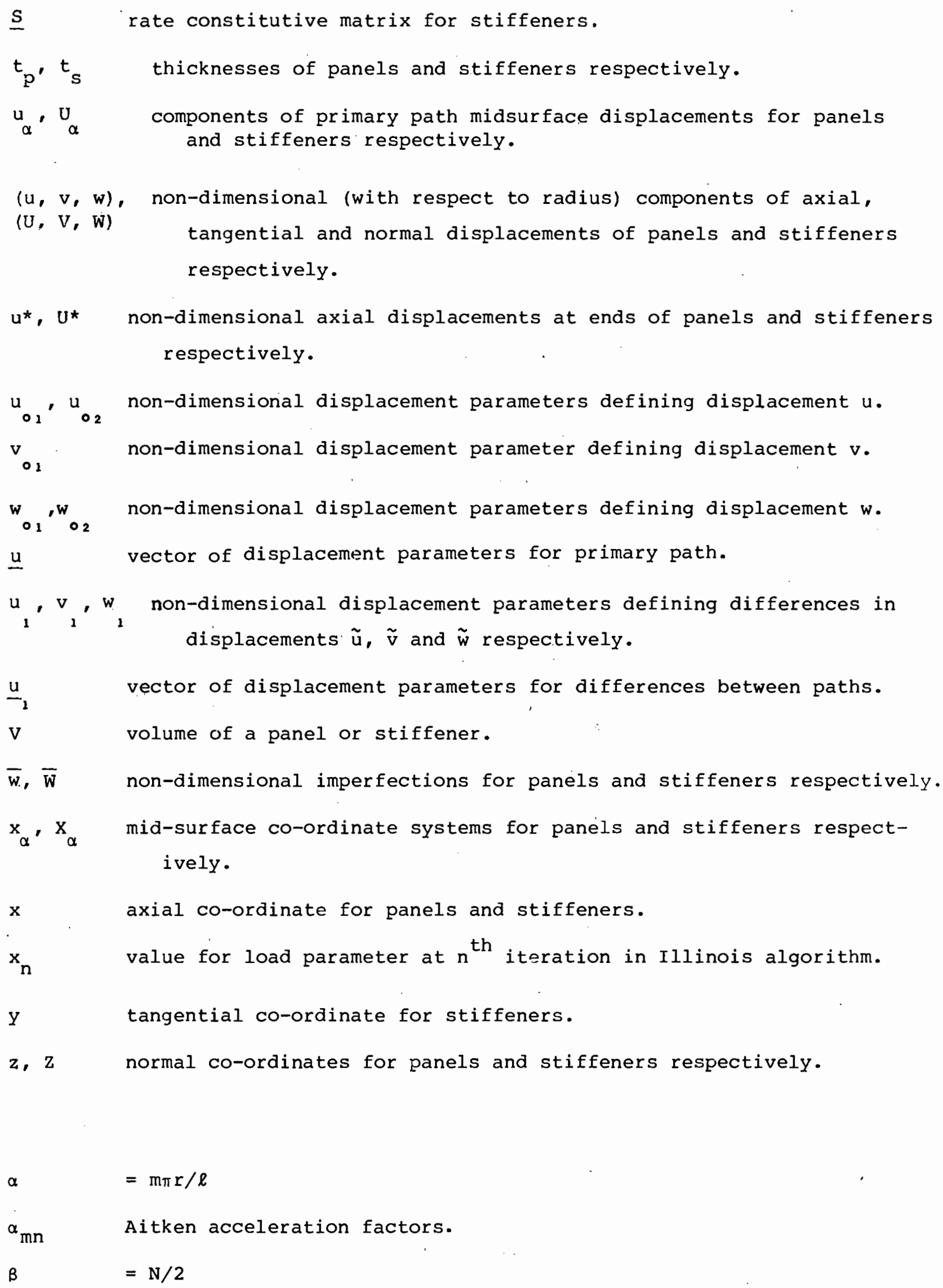


$\delta_{i j} \quad$ Kronecker delta

$\varepsilon_{\alpha \beta} \quad$ midsurface membrane strain tensor for panels.

$5 \quad=1+(y-e) / r$

$\eta_{i j} \quad$ three dimensional strain tensor.

$\eta_{i j}^{e}, \eta_{i j}^{p}$ three dimensional elastic and plastic components of strain tensor.

$\eta_{\alpha \beta} \quad$ strain tensor for plane stress, also strain tensor for panels.

$\bar{n} \quad$ effective strain.

$\vec{n}, \vec{\eta}$ elastic and plastic components of effective strain.

$\underline{n}$ strain vector for panels.

$\vartheta \quad$ "flatness"parameter for panels.

$\theta \quad$ circumferential (tangential) co-ordinate for panels.

$\theta_{0} \quad$ angular half-width of panels.

$x_{\alpha \beta} \quad$ midsurface bending strain tensor for panels.

$\mu \quad$ non-dimensional inperfection amplitude.

$\checkmark \quad$ Poisson's ratio.

$v \quad:=\frac{1}{2}+E_{s} / E\left(v-\frac{1}{2}\right)$

$\xi \quad=x / r$, non-dimensional axial co-ordinate.

o external axial compressive stress.

$\sigma_{c} \quad$ critical external axial compression stress (bifurcation stress).

$\sigma_{y} \quad y i e l d$ stress in simple tension.

$\sigma_{i j} \quad$ three dimensional stress tensor.

$\sigma_{\alpha \beta} \quad$ stress tensor for plane stress.

$\sigma_{i j}^{\prime} \quad$ stress deviator tensor. 
effective stress for uniaxial stress-strain curve.

plane stress vector for panels and stiffeners.

$\psi$

vector for five non-linear equations, obtained from the virtual

work formulation, that are solved in order to obtain a solution.

$(\ldots)^{p}$ pertaining to panel.

$(\ldots)^{\text {s }}$ pertaining to stiffener.

(...) increments in $(\ldots)$.

(..) difference for $(\ldots)$ between primary path and bifurcation path. 


\section{CONTENTS}

Page

Declaration $\ldots \ldots, \ldots \ldots, \ldots \ldots, \ldots \ldots, \ldots \ldots, \ldots \ldots \ldots$

Dedication

Acknowledgements

Abstract

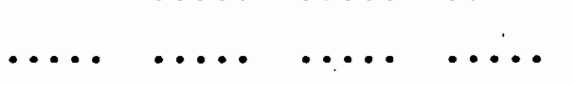

Notation

-...

$\cdots$

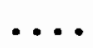

Contents

$$
\cdots
$$

-... $\ldots$

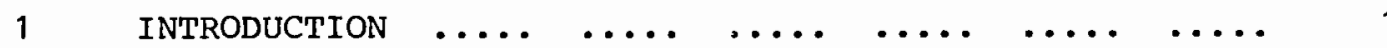

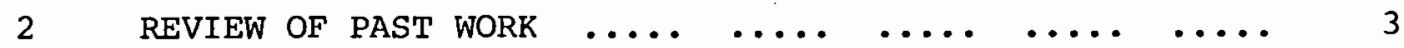

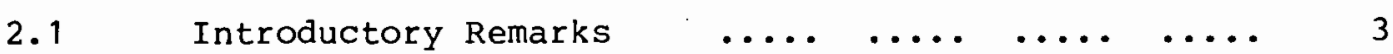

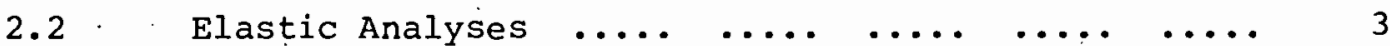

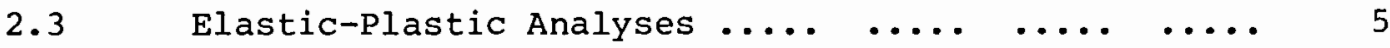

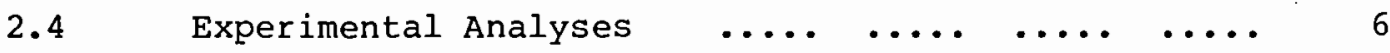

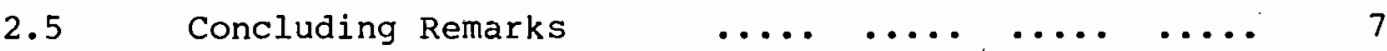

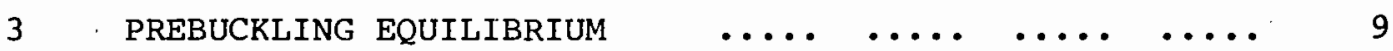

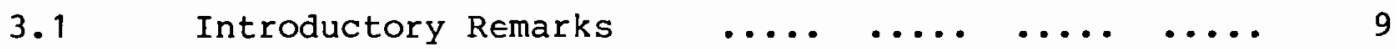

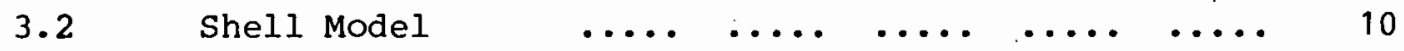

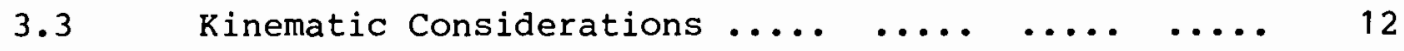

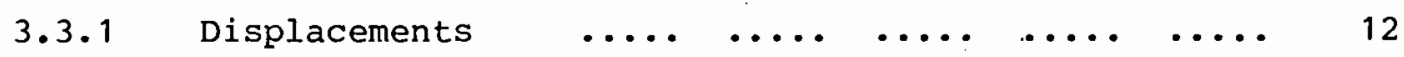

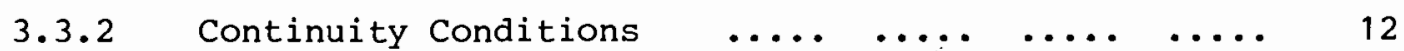

3.3.3 Strain-Displacement Relations $\ldots \ldots . \ldots . . . . .13$

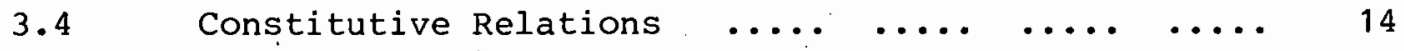

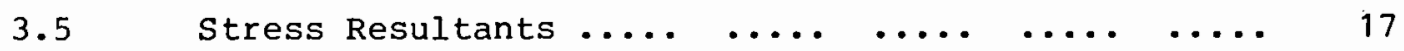

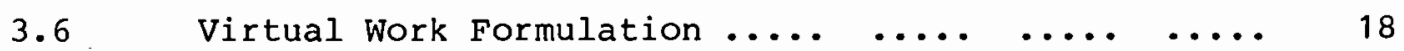

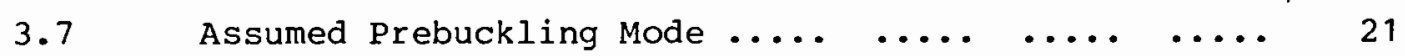

3.7.1 Assumed Displacement Functions $\ldots \ldots . \ldots . \ldots . . .21$

3.7.2 Complete Strains at a Point $\ldots \ldots . \ldots . \ldots . . .24$

3.7.3 Virtual Work Equations $\ldots \ldots . \ldots . . . . . .27$

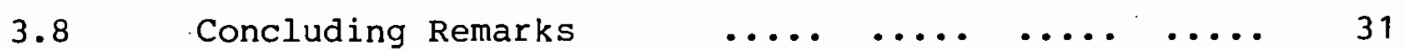

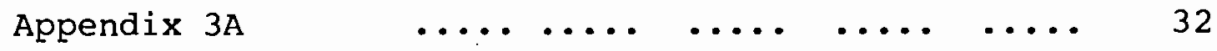

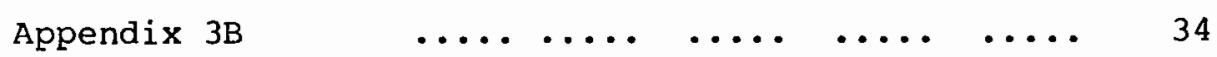

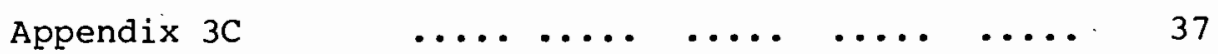

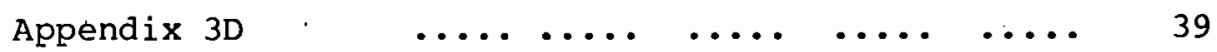

$4 / \quad \cdots+$ 
4 NUMERICAL PROCEDURES FOR THE PRIMARY EQUILIBRIUM PATH

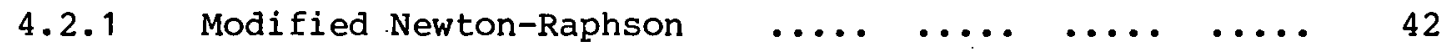

4.2.2 Aitken Acceleration .... .... .... .... .... 45

4.2.3 Automatic Increment Sizes .... .... .... ..... 48

4.2.4 Solution of Equations to Obtain Increments $\ldots . .48$

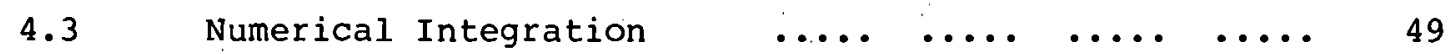

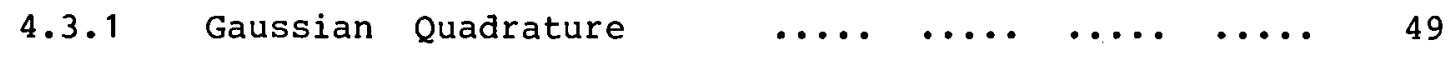

4.3.2 Integration over the Midsurface Areas $\ldots . . . . .50$

4.3.3 Integration Through the Thicknesses $\ldots \ldots \ldots . . .51$

4.4 Iteration for Secant Modulus f............ 54

4.5 Concluding Remarks $\ldots \ldots \ldots \ldots \ldots \ldots$

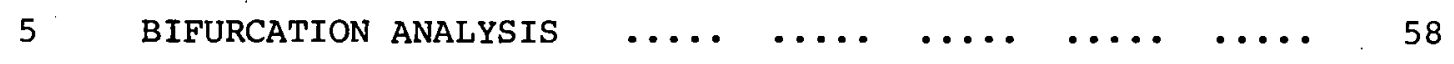

$5.1 \quad$ Introductory Renarks $\quad \ldots \ldots \ldots \ldots \ldots$

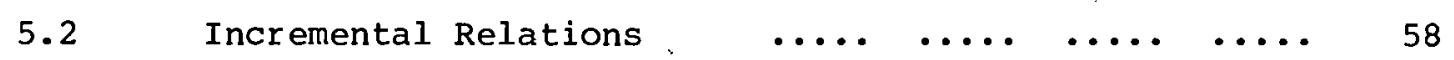

5.2.1 Incremental Strain-Displacement Relations $\ldots \ldots .58$

5.2 .2 Incremental Constitutive Relatịons.... .... .... 59

5.2 .3 Incremental Stress Resultants $\ldots . . \ldots \ldots . . . .66$

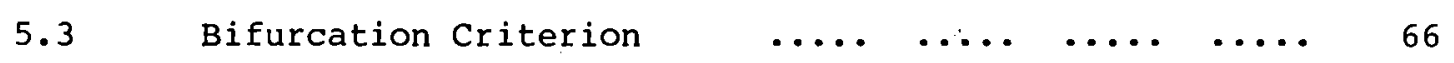

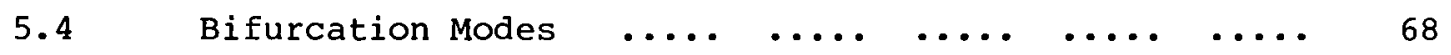

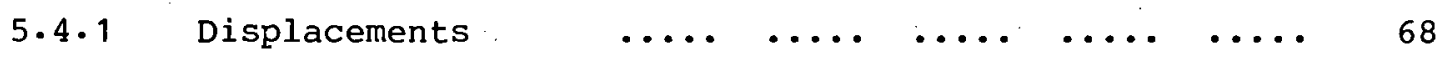

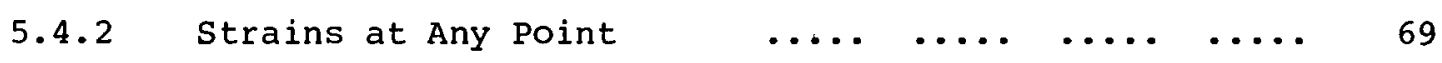

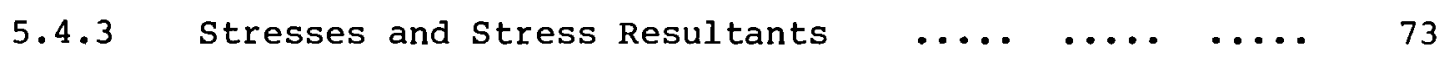

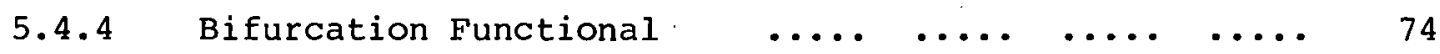

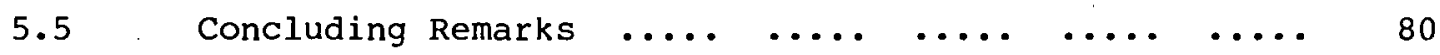

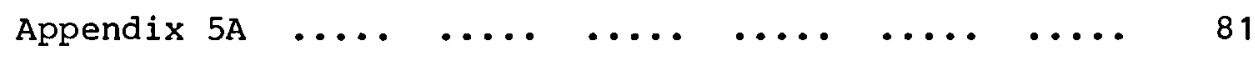

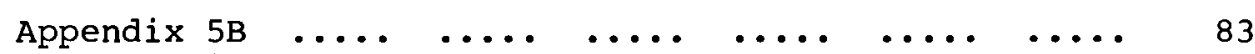

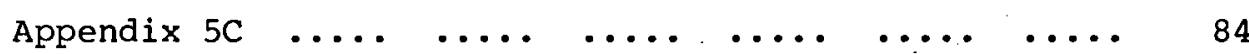

6 NUMERICAL PROCEDURES FOR BIFURCATION ANALYSIS $\ldots . .86$

6.1 Introductory Remarks $\quad \ldots \ldots \ldots \ldots$

6.2 Iteration for Bifurcation Load: Illinois Algorithm 86

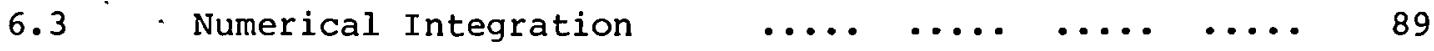


$\underline{\text { Page }}$

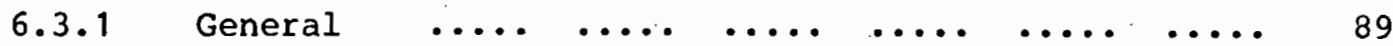

6.3.2 Numerical Integration over the Midsurface Areas 89

6.4 Concluding Remarks $\quad \ldots \ldots . \ldots . \ldots . \ldots . . . . .90$

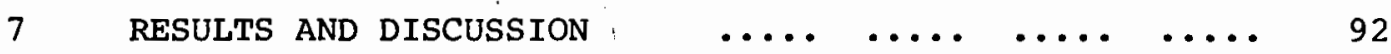

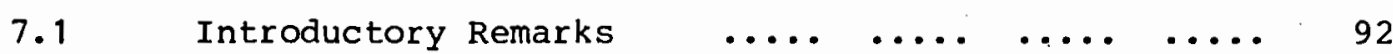

7.2 Comparison with Previous Results $\quad \ldots . . . . .992$

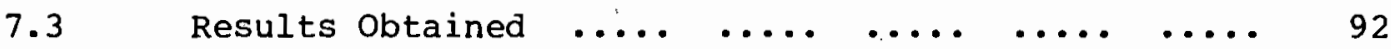

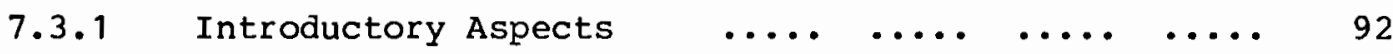

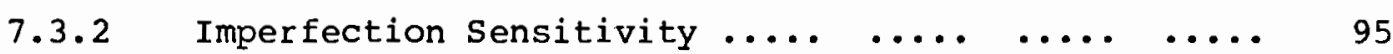

7.3 .3 Bifurcation Results $\quad \ldots \ldots . . . . . . . . . .106$

7.4 Comparison with Experimental Results ........ 109

7.5 Concluding Remarks $\quad \ldots \ldots \ldots \ldots \ldots 113$

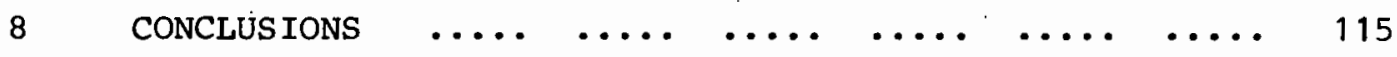

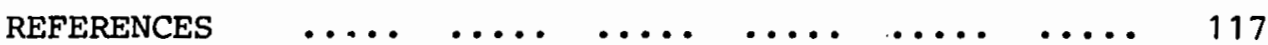




\section{CHAPTER 1}

INTRODUCTION

Interest in the behaviour of stiffened cylinders in axial compression has been generated by their use as components of large off-shore structures and also for aero-space structures. Much work on cylinder buckling has been carried out to determine the behaviour of aero-space structures which generally have relatively thin walls and closely spaced stiffeners with high torsional rigidity. In contrast, off-shore structures are generally sparsely stiffened and the stiffeners are sufficiently flexible torsionally to be able to twist along their length. These marine structures are very susceptible to geometric imperfections during their fabrication and also to damage while they are being transported. These effects can alter the behaviour of stiffened cylinders in service and their consequences are thus of particular interest, with regard to buckling in axial compression.

Stiffened cylinders that are used for aero-space applications have narrow panels which have a low "flatness" parameter as described by Koiter (1956). Due to the high torsional rigidity of the stiffeners and the "flatness" of the panels these cylinders tend to buckle into an overall mode in which one half-wave in the circumferential direction may extend across a number of stiffeners. These critical bifurcation stresses are generally calculated by "smearing-out" the stiffener properties to obtain an equivalent or thotropic cylinder of uniform thickness (Hutchinson and Amazigo (1967)). Broad-panelled cylinders such as those used for marine structures have a high "flatness" parameter and cannot be analysed successfully in this way. It is thus necessary to consider the panels and stiffeners as two separate structural components with the interactions between the two coupled by continuity conditions of displacements and equilibrium conditions along the intersecting lines of the stiffeners and the shell. In this type of analysis the panels are treated as cylindrical panels and the stiffeners may be treated in various ways, the most recent being the approach by Syngellakis and walker (1978a, 1978b) who have treated the stiffeners as flat plates. A review of past work in these fields can be found in Chapter 2 .

The present analysis is essentially an extension of the work done by Syngellakis and walker (1978b) into the plastic range. The analysis is concerned with the effects of initial imperfections on the general behaviour 
and buckling of simply supported stiffened cylindrical shells. These shells are of the type found in marine structures and are analysed under an axial compressive load. There are an even number of equally spaced stiffeners which are treated as flat plates. The complexity of the shell geometry and the vast number of parameters prevents a complete investigation so that the discussion of results will centre around the behaviour of a shell with internal stiffeners, and the effect of different panel thicknesses. The imperfections are assumed to have the approximate shape of the buckling mode of a perfect sparsely-stiffened cylinder in axial compression. To assess the effects of these imperfections it is necessary to obtain the load-deformation behaviour of the shell in the elastic and plastic ranges. The equations used to determine this load-deformation behaviour, or the primary equilibrium path as it is called, are developed in Chapter 3 with their numerical implementation described in chapter 4. Small-strain $J_{2}$ deformation theory of plasticity is used in the formulation of these equations.

The shell deforms in the same shape as the buckling mode of the perfect stiffened cylinder, the mode being symmetrical in the circumferential direction about the mid-generator of the panel. The shell is now capable of bifurcating into an anti-symmetric mode with respect to the mid-generator of the panel. The critical bifurcation stresses associated with these buckling modes are sought in the second part of the analysis.: The equations used to locate these bifurcation points are developed in Chapter 5 with their numerical implementation shown in Chapter 6 . The results obtained by this analysis are discussed in Chapter 7 where an attempt is made to compare the theoretical results with those obtained from experiments. 
CHAPTER 2

\section{REVIEW OF PAST WORK}

\section{$2.1 \quad$ Introductory Remarks}

A brief review of the analyses leading up to the present analysis is presented in this chapter. Elastic analyses are reviewed in section 2.2 which includes work by Koiter (1956) and Stephens (1971) on cylindrical panels and work on stiffened cylinders by Hutchinson and Amazigo (1967), Wang and Lin (1973) and Syngellakis and Walker (1978a, 1978b). In Section 2.3 the elasticplastic analyses of Tvergaard (1977) on cylindrical panels and of Reddy (1980) on stiffened shells in axial compression are presented. Experimental work by Walker and Davies (1976) is discussed in Section 2.4. Experimental results in other papers by Walker and co-authors are also noted.

\subsection{Elastic Analyses}

Koiter (1956) considered the buckling and post-buckling behaviour of a cylindrical panel in axial compression. This type of panel occurs between stiffeners of a sparsely stiffened cylinder where the critical mode in the circumferential direction is likely to be a local mode between the stiffeners. The problem was confined to the elastic range and it was assumed that the stiffeners have no torsional rigidity and suppress the radial deflection waves along their lines of attachment. Koiter introduced a "flatness" parameter which is given by

$$
\vartheta=\stackrel{4}{r}\left[12\left(1-v^{2}\right)\right] \stackrel{\theta_{0}}{\pi} r(r / t)
$$

where $\mathrm{r} / \mathrm{t}$ is the shell radius/thickness ratio, $\theta_{0}$ is half the angle between two stiffeners and $v$ is Poisson's ratio. Koiter found that for sufficiently flat panels which have $\vartheta$ less than 0,64 the post-buckling behaviour was stable while more curved panels with $\vartheta$ larger than this value had unstable post-buckling behaviour. The effect of initial imperfections in the panel was also investigated and it was found that the critical load was lowered with an increase in imperfection.

Hutchinson and Amazigo (1967) presented a study of the imperfection sensitivity of eccentrically stiffened shells under axial compression. The 
calculations carried out were based on "smeared-out" stringer properties which give an equivalently orthotropic shell and, as with Koiter, the torsional stiffness of the stiffeners was therefore neglected. As with Koiter (1956), initial imperfections in the form of a buckling mode of the perfect shell were considered. The results presented show that stiffening on the outside of the cylinder produces higher buckling loads than stiffeners to the inside, but that outside stiffening is generally more imperfection sensitive.

Stephens (1971) extended Koiter's (1956) analysis and considered the buckling and post-buckling behaviour of an axially compressed cylindrical panel taking the torsional rigidity of the stringers into account. This was done by setting the bending moment at the longitudinal boundaries of the panel equal to the torsional moment in the stiffener. There was no twisting of the stiffeners in the prebuckling state since deformation was symmetric, but this boundary condition allowed the stiffeners to twist along their lengths in the buckled state. Stephens was then able to show that the inclusion of the torsional rigidity increased the critical stress of a panel and also decreased the imperfection sensitivity. This indicates that stable post-buckling behaviour is possible for wider panels between stiffeners than Koiter (1956) anticipated. Thus the value of the "flatness" parameter $\vartheta$, below which stable post-buckling behaviour occurs, can be increased. The effect of internal pressure was also included in this analysis and it was shown that this also reduced the sensitivity of the panel to imperfections and increased the critical stress.

Wang and Lin (1973) approached the buckling problem of a discretely stiffened cylinder in axial compression by treating the stiffeners, and the panels between adjacent stiffeners, as separate structural components. Donnell's shell theory was used for the panels and vlascv's thin-walled beam theory was used for stiffeners of arbitrary cross-section. The interactions between the panels and the stiffeners were coupled through continuity conditions of displacements and equilibrium conditions for stresses along the intersecting lines of the stiffeners and the shell. Simply supported cylinders were analysed and it was found that the stiffeners twisted along their length but had negligible transverse deflection due to bending. Critical loads obtained in this analysis were found to be lower than those obtained using the "smeared" analysis especially for short shells. In agreement with Hutchinson and Amazigo' (1967), critical loads for cylinders with external stiffeners were found to be higher than those with internal stiffeners. It was also found that cylinders with fully clamped end conditions also had 
higher critical stresses than the simply supported cylinders.

Syngellakis and walker (1978a) approached the problem of buckling of stiffened cylinders by treating the stiffeners as long narrow plates. Assumptions, consistent with experimental observations, governing the choice of the displacement functions, simplified the solution procedure. It was shown that these stiffened cylinders had several critical loads in close proximity, which could result in a coupling of modes in the post-critical range and thus lead to an increase in imperfection-sensitivity. Initial post-critical paths for each critical stress of a particular shell were found to be either stable or unstable, depending on the shell geometry. Experimenal data for one shell was compared with the theoretical analysis for a particular shell geometry. All the lower bifurcation loads individually were found theoretically to be stable and the mode with the steepest post-critical. tangent coincided with the shape of the mid-panel deformation at an advanced stage of loading as found in the experimental analysis. This was found helpful when chosing the predominant imperfection amplitude in the bifurcation analysis of an imperfect cylinder.

In the second report, Syngellakis and walker (1978b) extended their analysis by considering the effects of geometric imperfections in the shape of the mode referred to above. This mode was assumed to be symmetric about a panel mid-generator and antisymmetric about a stiffener. The imperfection was thus assumed to be of the same form and its effect on the buckling of the cylinder into an antisymmetric mode with respect to the panel mid-generator was considered. The critical loads for this antisymmetric mode were found to be higher than those obtained in the previous analyses although there were still several critical loads in close proximity. It was noted by Syngellakis and Walker that only one of many components of imperfection was accounted for.

\section{$2.3 \quad$ Elastic-Plastic Analyses}

There is a limited amount of work related to buckling of stiffened cylinders in the plastic range. Tvergaard (1977) extended Koiter's (1956) analyses of a simply supported axially compressed cylindrical panel into the plastic range. This analysis was concerned with the local buckling of the panels between stiffeners of sparsely stiffened shells. The stiffeners were assumed to remain straight and their torsional rigidity was neglected. Bifurcation stresses were determined analytically and an asymtotically exact expression/ $\quad$..... 
expression was obtained for the initial post-bifurcation behaviour in the plastic range. Imperfection-sensitivity was analysed on the basis of $J_{2}$ flow theory with elastic unloading neglected, as well as by a numerical study . It was found that for a low hardening material the panel was imperfection-sensitive for all curvatures considered but that for a high hardening material the panel was only imperfection-sensitive if the curvature exceeded a certain value.

Reddy (1980) extended Syngellakis and Walker's (1978a) analysis of a simply supported cylindrical shell with an even number of stiffeners into the plastic range. Both $\mathrm{J}_{2}$ flow and deformation theories of plasticity were used. The results presented show the effects of the stiffener size on the critical stresses for both elastic and elastic-plastic shells with internal stiffeners. It was also shown that when the critical stress of a stiffener with low torsional rigidity, treated as a long plate simply supported on three sides and free along one longitudinal edge, is much lower than the critical stress of a simply supported cylindrical panel then the critical stress of the composite shell can be significantly reduced below the critical stress of the panel. This is contrary to the implications of Koiter (1956) and Tvergaard (1977) that shells having stiffeners with low torsional rigidity may be treated as an assembly of simply supported panels. It was also shown that when the torsional rigidity of the stiffeners is high then the critical stress of the composite shell can be increased by making the panels narrower (increasing the number of stiffeners). Discrepancies in critical stresses obtained by using flow and deformation theories of plasticity were also discussed .

\subsection{Experimental Analyses}

The only experimental work related to stiffened cylinders in axial compression appears to be that of walker and his co-workers. In the experimental report by Walker and Davies (1976) a series of tests on stiffened steel cylinders under axial compression was carried out. Small scale steel models were built by welding rolled panels and stiffeners together; the shell was then bolted into the testing machine to provide a fully clamped end condition. Two series of specimens were tested and the load versus end-shortening behaviour was obtained. These cylinders had initial geometric imperfections which were measured and given in terms of three parameters for the cylinders tested. A comparison of experimental and theoretical failure loads from 
design rules was also included.

Syngellakis and Walker (1978a, 1978b) tested an araldite stiffened cylinder in order to compare the results obtained from their analysis. This cylinder was simply supported and the load versus end-shortening behaviour was obtained. The initial imperfection was again determined and used in their analysis. A number of experimental results for fixed ended cylinders are included in the analysis of stiffened cylinders by Walker and Sridharan (1980).

\subsection{Concluding Remarks}

A brief review of the work to date on stiffened cylinders in axial compression has been presented. This traces from Koiter's (1956) analysis of an elastic panel up to Reddy's (1980) analysis of an elastic-plastic stiffened cylinder and Syngellakis and Walker's (1978b) analysis of an imperfect elastic stiffened cylinder. A review of experimental work has also been presented with which the results from the present analysis will be compared in a later chapter. The analysis of an imperfect elastic-plastic stiffened cylinder is presented in the following chapters. The path linking these analyses can best be seen in Fig 2.1. 


\begin{tabular}{|c|}
\hline $\begin{array}{c}\text { Elastic panel } \\
\text { - imperfection sensitivity } \\
\text { KOI TER (1956) }\end{array}$ \\
\hline $\begin{array}{l}\text { Elastic stiffened shell } \\
\text { - 'smeared' analysis } \\
\text { - imperfection sensitivity } \\
\text { HUTCHINSON, AMAZIGO (1967) }\end{array}$ \\
\hline
\end{tabular}

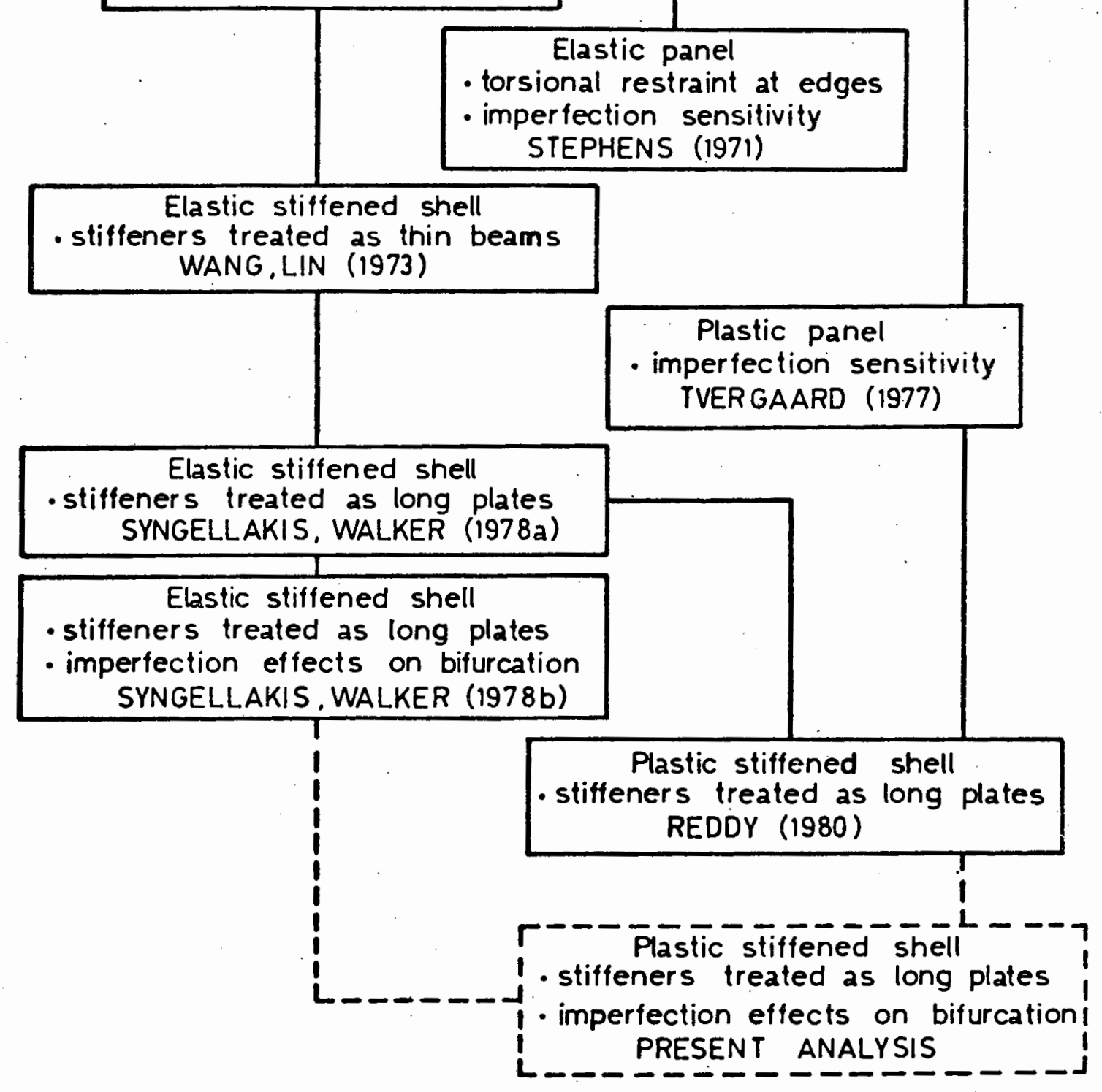

Experimental results

- clamped ended shells : WALKER, DAVIES (1976)

- simply supported shells : SYGELLAKIS,WALKER (1978a, 1978b)

Fig. 2.1 Flow Chart of Analyses to date. 


\section{CHAPTER 3}

\section{PREBUCKLING EQUILIBRIUM}

\subsection{Introductory Remarks}

In this chapter, we set up the problem of determining the primary equilibrium path for an axially compressed circular cylinder with longitudinal stiffeners which has an initial stress-free imperfection. The shell model is discussed in section 3.2, and the non-linear strain-displacement relations are presented in section 3.3 for the midsurfaces of the panels and the stiffeners, the latter being considered as flat plates. Using these relations it is possible to obtain the strains at any point in the thickness of the panels and stiffeners.

The stresses corresponding to these strains are determined by the constitutive equations in section 3.4. The constitutive equations used correspond to small strain $J_{2}$ deformation theory of plasticity. The bending moment and membrane stress resultants are obtained in section 3.5 and used, together with the strain-displacement relations, to formulate the principle of virtual work for the stiffened shell.

The assumed imperfection and displacements of the panels are described in Section 3.7 as sinusoidal functions of the midsurface coordinates. The corresponding stiffener imperfection and displacement are then derived in the same section. Substitution of these results in the equations for virtual work then leads to a formulation of the problem entirely in terms of displacement quantities. 


\subsection{Shell Model}

The shell considered comprises a circular cylinder with equally spaced longitudinal or stringer stiffeners, as shown in Fig. 3.1. The cylinder has length $\ell$, radius $r$ and wall thickness $t_{p}$. There are an even number, $N$, of stiffeners which have depth $2 d$ and thickness $t_{s}$. Each panel has angular width $2 \theta_{0}$ where

$$
\theta_{0}=\pi / \mathrm{N}
$$

Points on the midsurface of the $i^{\text {th }}$ panel are defined by a pair $\left(x, r \theta^{(i)}\right.$ ) or $\left(x_{1}, x_{2}\right)$ of cylindrical coordinates while points on the midsurface of the $i^{\text {th }}$ stiffener, considered to be a flat plate, are defined by a pair $(x, y)$ or $\left(x_{1}, x_{2}\right)$ of cartesian coordinates. Distances normal to the panel midsurface are measured by the coordinate $z$, while distances normal to the stiffener midsurface are measured by the coordinate $z$. These coordinate systems are also shown in Fig. 3.1. The superscript (i) denoting the $i^{\text {th }}$ panel or stiffener is omitted when referring to any typical panel or stiffener.

The centroids of the stiffeners are a distance $y=e$ from the midsurface of the shell, so that for an internally stiffened shell

$$
e=d-t_{p} / 2
$$




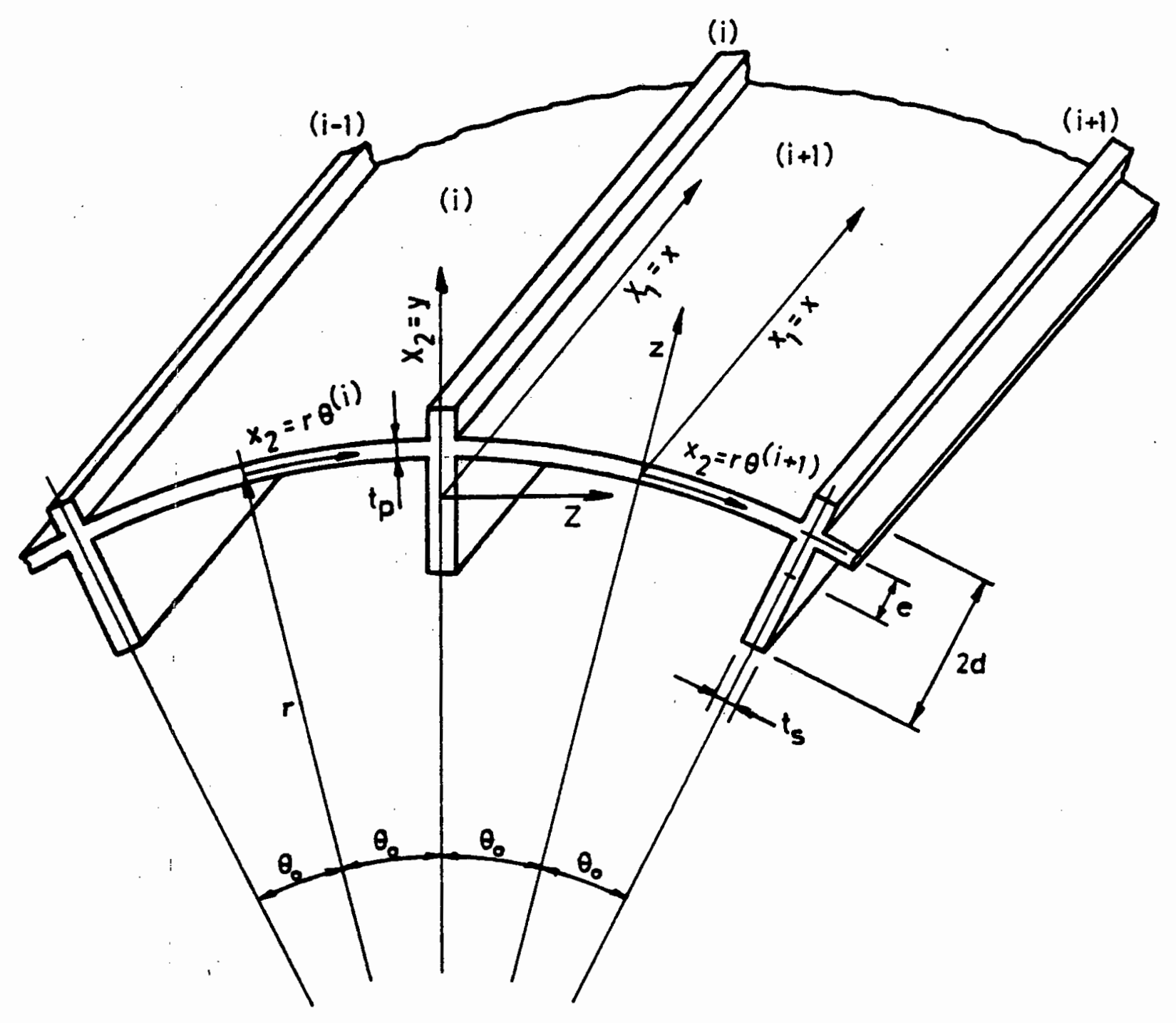

Fig. 3.1 Geometry of Stiffened Cylinder 


\subsection{Kinematic Considerations}

\subsubsection{Displacements}

Panel and stiffener displacements are defined, respectively, by

$$
\begin{aligned}
& \underline{u}=u_{\alpha} \underline{e}_{\alpha}+w \underline{e}_{3} \\
& \underline{U}=u_{\alpha} \underline{g}_{\alpha}+\underline{w g}_{3}
\end{aligned}
$$

where the $\underline{e}_{i}$ and $\underline{g}_{i}$ are unit base vectors for the panels and stiffeners, respectively. Greek subscripts range from 1 to 2 and summation is implied over repeated indices here and elsewhere.

The initial stress-free imperfection of the panels, which is a sinusoidal function of $\mathbf{x}$ and $\theta$, is denoted by $\bar{w}$. This imperfection is a radially outward or inward initial deviation from the perfect cylindrical shell. An imperfection $\bar{W}$ in the stiffeners is associated with the panel imperfection, as will be shown later.

\subsubsection{Continuity Conditions}

A fundamental requirement is that of continuity of displacements and slopes at the $i^{\text {th }}$ panel-stiffener junction. Using the compatibility conditions of Syngellakis and walker (1978a) and introducing the imperfections, we obtain the following conditions:

$$
\begin{aligned}
& u^{(i)}\left(x, \theta_{0}\right)=u^{(i)}(x, e)=u^{(i+1)}\left(x,-\theta_{0}\right) \\
& v^{(i)}\left(x, \theta_{0}\right)=w^{(i)}(x, e)=v^{(i+1)}\left(x,-\theta_{0}\right) \\
& w^{(i)}\left(x, \theta_{0}\right)=v^{(i)}(x, e)=w^{(i+1)}\left(x,-\theta_{0}\right)
\end{aligned}
$$

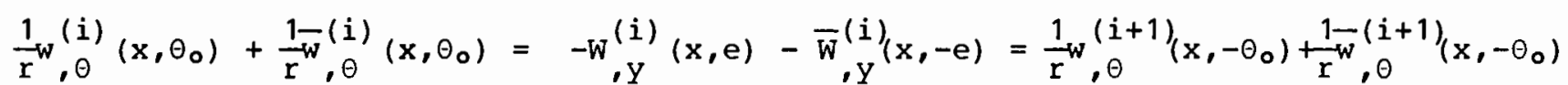

This last equation imposes the condition that the stiffener cross-sections remain normal to the shell. Assuming that the stiffener cross-sections also remain straight the stiffener displacements can be written in terms of the panel displacements as follows:

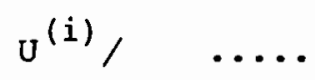




$$
\begin{aligned}
& U^{(i)}(x, y)=u^{(i)}\left(x, \theta_{0}\right)-(y-e) w_{, x}^{(i)}\left(x, \theta_{0}\right) \\
& v^{(i)}(x, y)=\zeta w^{(i)}\left(x, \theta_{0}\right) \\
& w^{(i)}(x, y)+\bar{w}^{(i)}(x, y)=v^{(i)}\left(x, \theta_{0}\right)-\frac{1}{r}(y-e)\left[w_{,}^{(i)}\left(x, \theta_{0}\right)+\vec{w}_{, \theta^{(i)}}^{\left.\left(x, \theta_{0}\right)\right]}\right. \\
& \text { where } \zeta=1+\frac{1}{r}(y-e) . \quad \text { This factor is discussed in section } 3.7 .1 .
\end{aligned}
$$

\subsubsection{Strain-displacement relations}

The strain-displacement relations for thin plates and shells are used, taking into account the presence of the imperfection. In particular, the Donnell-Mushtari-Vlasov equations (Sanders (1963)) for quasi-shallow shells are appropriate for the present problem. The imperfection amplitude is in general much smaller than the shell thickness, and thus terms involving squares of the imperfection are neglected in the strain-displacement equations; these equations are then, for a typical panel,

$$
\begin{gathered}
\varepsilon_{\alpha \beta}\left(x_{1}, x_{2}\right)=\frac{1}{2}\left(u_{\alpha, \beta}+u_{\beta, \alpha}\right)-b_{\alpha \beta} w+\frac{1}{2}\left(w, \alpha{ }_{, \beta}+w_{, \alpha}{ }^{w}, \beta+{ }_{, \alpha}{ }^{\bar{w}}, \beta\right) \\
x_{\alpha \beta}\left(x_{1}, x_{2}\right)=w_{, \alpha \beta},
\end{gathered}
$$

Here $\epsilon_{\alpha \beta}$ and $x_{\alpha \beta}$ are the midsurface membrane and bending strain tensors and $b_{\alpha \beta}$ is the curvature tensor for the undeformed midsurface $\left(b_{22}=-1 / r\right.$, others are zero $)$. The midsurface membrane and bending strain tensors for a typical stiffener are

$$
\begin{gathered}
E_{\alpha \beta}\left(X_{1}, X_{2}\right)=\frac{1}{2}\left(U_{\alpha, \beta}+U_{\beta, \alpha}\right)+\frac{1}{2}\left(W_{, \alpha}{ }_{, \beta}+W_{, \alpha} \bar{W}_{, \beta}+W_{, \beta} \bar{W}_{, \alpha}\right) \\
K_{\alpha \beta}\left(X_{1}, X_{2}\right)=W_{, \alpha \beta}
\end{gathered}
$$

Note that the membrane strain tensors are zero when only the imperfection is present. The strain tensors at any point through the thickness of a typical panel and stiffener are given by

$$
\begin{aligned}
& \eta_{\alpha \beta}\left(x_{1}, x_{2}, z\right)=\varepsilon_{\alpha \beta}-z x_{\alpha \beta} \\
& H_{\alpha \beta}\left(x_{1}, X_{2}, z\right)=E_{\alpha \beta}-z K_{\alpha \beta}
\end{aligned}
$$




\subsection{Constitutive Relations}

The theory of plasticity employed here is small strain $\mathrm{J}_{2}$ deformation theory. This is a total strain theory, i.e. the stresses are directly related to the total strains at that point during the loading. This theory works well for proportional or nearly proportional loading (Budiansky(1959)), that is under conditions where the stress-component ratios are nearly constant during the loading (Malvern(1969)), i.e.

$$
\frac{\sigma_{11}}{\sigma_{11}^{0}} \simeq \frac{\sigma_{22}}{\sigma_{22}^{0}} \simeq \ldots \simeq \frac{\sigma_{12}}{\sigma_{12}^{0}}
$$

where $\sigma_{i j}^{\circ}$ are the stress values at initial yield. For the present problem it is expected that the deviation from proportional loading will be small, and so it is reasonable to use $\mathrm{J}_{2}$ deformation theory. Furthermore, for problems involving thin shells, deformation theory is known to yield results which are generally in better agreement with experimental data, than results obtained using flow theory.

The total strain at any point in a continuum can thus be written as the sum of an elastic strain $\eta_{i j}^{e}$, and a plastic strain $\eta_{i j}^{p}$, as follows:

$$
n_{i j}=n_{i j}^{e}+n_{i j}^{p}
$$

Here, the elastic component of strain is given by

$$
\eta_{i j}^{e}=\frac{1+u}{E} \sigma_{i j}-\frac{u}{E} \sigma_{k k} \delta_{i j}
$$

which is the usual isotropic Hooke's law with Young's modulus $E$ and Poisson's ratio $v, \delta_{i j}$ being the kronecker delta. The plastic component of strain is given by (Malvern (1969))

$$
n_{i j}^{p}=\frac{3 \bar{\eta}}{2 \bar{\sigma}}\left(\sigma_{i j}-\frac{1}{3} \sigma_{k k} \delta_{i j}\right)
$$

where $\bar{\eta} p$ is the effective plastic strain; $\vec{\sigma}$ is the effective stress, 
defined by

$$
\bar{\sigma}=\sqrt{3} J_{2},
$$

where

$$
J_{2}=\frac{1}{2} \sigma_{i j} \sigma_{i j}
$$

and

$$
\sigma_{i j}=\sigma_{i j}-\frac{1}{3} \sigma_{k k} \delta_{i j}
$$

is the stress deviator tensor. In the case of approximate plane stress the effective stress reduces to

$$
\bar{\sigma}^{2}=\sigma_{11}^{2}+\sigma_{22}^{2}-\sigma_{11} \sigma_{22}+3 \sigma_{12}^{2} .
$$

The effective stress and total effective strain are assumed to be related in the same way as stress and strain in a uniaxial test. The secant modulus $E_{s}$ is then defined as the effective stress $\bar{\sigma}$, divided by the total effective strain $\bar{\eta}$ which comprises an elastic component $\vec{\eta}$, and a plastic component $\vec{\eta}^{p}$. It follows that

$$
\frac{1}{E_{s}}=\frac{\bar{n}}{\bar{\sigma}}=\frac{\vec{n}^{e}+\vec{n}^{p}}{\bar{\sigma}}=\frac{1}{E}+\frac{\vec{n}^{p}}{\bar{\sigma}} .
$$

Using this relation, equation $(3.15)$ becomes

$$
\eta_{i j}^{p}=\frac{3}{2}\left(\frac{1}{E_{s}}-\frac{1}{E}\right)\left(\sigma_{i j}-\frac{1}{3} \sigma_{k k} \delta_{i j}\right)
$$

Combining the elastic and plastic components of strain and rearranging, we obtain, for the total strain, the expression

$$
\eta_{i j}=\frac{1+v_{s}}{E_{s}} \sigma_{i j}-\frac{v_{s}}{E_{s}} \sigma_{k k} \delta_{i j}
$$

with

$$
u_{S}=\frac{1}{2}+\frac{E_{S}}{E}\left(u-\frac{1}{2}\right)
$$

It is seen that the strain-stress relations have the same form as those in Hooke's law (3.14) but the coefficients are altered due to the degree of 
plasticity encountered.

The following uniaxial stress-strain curve is adopted here:

$$
\begin{aligned}
\bar{n} & =\frac{\bar{\sigma}}{E} & & \text { for } \sigma \leqslant \sigma_{y} \\
& =\frac{\sigma}{E}\left[\frac{1}{n}\left(\frac{\bar{\sigma}}{\sigma_{y}}\right)^{n}+\frac{n-1}{n}\right] & & \text { for } \sigma>\sigma_{y} .
\end{aligned}
$$

Where $\sigma_{y}$ is the yield stress of the material considered and $n$ is the strain hardening parameter. It must be noted that the first derivative or tangent modulus is continuous at the yield stress and that by setting $\mathrm{n}=1$ the second equation reduces to the first, thus being suitable for elastic material behaviour. This stress-strain curve was first suggested by Needleman (1975). The secant modulus can now be determined as

$$
\begin{aligned}
\frac{1}{E_{S}}=\frac{\bar{n}}{\bar{\sigma}} & =\frac{1}{E} & & \text { for } \sigma \leqslant \sigma_{y} \\
& =\frac{\sigma}{\bar{\sigma} E}\left[\frac{1}{n}\left(\frac{\bar{\sigma}}{\sigma_{y}}\right)^{n}+\frac{n-1}{n}\right] & & \text { for } \sigma>\sigma_{y} .
\end{aligned}
$$

Inversion of equation (3.18) results in the following constitutive equation

$$
\sigma_{i j}=\bar{c}_{i j k \ell \ell_{k \ell}}
$$

assuming that a state of approximate plane stress prevails in the panels and stiffeners, we may write this equation in the form

$$
\sigma_{\alpha \beta}=C_{\alpha \beta \mu \gamma}{ }^{n} \mu \gamma
$$

where $\alpha$ and $\beta$ range from 1 to 2 and the coefficients $C_{\alpha \beta \mu \gamma}$ are found by

$$
C_{\alpha \beta u \gamma}=\bar{C}_{\alpha \beta \mu \gamma}-\frac{\bar{C}_{\alpha \beta 33} \bar{C}_{33 \mu \gamma}}{\bar{C}_{3333}} .
$$

This relation is derived in Appendix $3 \mathrm{~A}$. 
Inversion of equation (3.18)and the use of (3.24) enables equation (3.23) to be written in the form

$$
\underline{\sigma}^{p}=\underline{c} \underline{n}
$$

for a typical panel and

$$
\underline{\sigma}^{s}=\underline{C} \underline{H}
$$

for a typical stiffener. Here,

$$
\begin{aligned}
& \underline{\sigma}^{p}=\left(\sigma_{x x}, \sigma_{\theta \theta}, \sigma_{x \theta}\right)^{T}, \\
& \underline{\sigma}^{s}=\left(\sigma_{x x}, \sigma_{y y}, \sigma_{x y}\right)^{T}
\end{aligned}
$$

and

$$
\underline{C}=\frac{E_{s}}{\left(1-\nu_{s}^{2}\right)}\left[\begin{array}{ccc}
1 & \nu_{s} & 0 \\
\nu_{s} & 1 & 0 \\
0 & 0 & 1-\nu_{s}
\end{array}\right]
$$

\subsection{Stress Resultants}

The bending moment and stress resultant tensors for a typical panel and stiffener are defined by

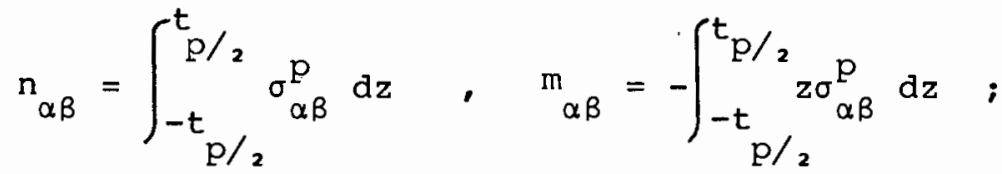

$$
\begin{aligned}
& N_{\alpha \beta}=\int_{-t}^{t s / 2} \sigma_{S / 2}^{s} d z \quad, \quad M_{\alpha \beta}=-\int_{-t s_{s / 2}^{t} s / 2}^{t_{\alpha \beta}} 2 \sigma_{\alpha \beta} d z
\end{aligned}
$$

The column vectors of membrane and bending stress resultants for a typical panel and stiffener are defined by

$$
\underline{r}=\int_{-t}^{t} \frac{\underline{D}^{p}}{p / 2} \underline{\sigma}^{p} d z,
$$




$$
\underline{R}=\int_{-t_{S / 2}}^{t} s / 2 \text { D } \underline{\sigma}^{s} d z
$$

where

$$
\begin{aligned}
& \underline{r}^{T}=\left(n_{x x}, n_{\theta \theta^{\prime}} n_{x \theta^{\prime}} m_{x x}, m_{\theta \theta}, m_{x \theta}\right), \\
& \underline{R}^{T}=\left(N_{x x}, N_{y y^{\prime}} N_{x y}, M_{x x}, M_{y y}, M_{x y}\right), \\
& \underline{D}^{p}=\left[\begin{array}{lll}
1 & 0 & 0 \\
0 & 1 & 0 \\
0 & 0 & 1 \\
-z & 0 & 0 \\
0 & -z & 0 \\
0 & 0 & -z
\end{array}\right], \quad \underline{D}^{s}=\left[\begin{array}{lll}
1 & 0 & 0 \\
0 & 1 & 0 \\
0 & 0 & 0 \\
-z & 0 & 0 \\
0 & -z & 0 \\
0 & 0 & -z
\end{array}\right]
\end{aligned}
$$

\subsection{Virtual Work Formulation}

For small strains and stresses that are, in general, much smaller than the Young's modulus of the material, the following three dimensional form of the principle of virtual work are valid

$$
\int_{V} \sigma_{i j} \delta \eta_{i j} d v=\int_{S} F_{i} \delta u_{i} d S
$$

where $\sigma_{i j}$ is the Cauchy stress tensor, $\eta_{i j}$ is the Lagrangian strain tensor and the $F_{i}$ and $u_{i}$ are the components of surface traction and displacement on the boundary. Integrals are over the original volume and surface area. In the present context the above equation reduces to

$$
\sum_{i=1}^{N}\left\{\int_{V} p(i){ }_{\alpha \beta}^{\sigma^{p}} \delta n_{\alpha \beta} d v^{p(i)}+\int_{V} s(i){ }_{\alpha \beta}^{s} \delta H_{\alpha \beta} d v^{s(i)}\right\}=\delta w_{e x t}^{p}+\delta w_{e x t}^{s}
$$

where the right hand side is usually known as the external virtual work and the left hand side the internal virtual work. By using equations (3.11) and (3.12) as well as the bending moment and stress resultant tensors as given in 
equations (3.27) and (3.28) we obtain the following equation:

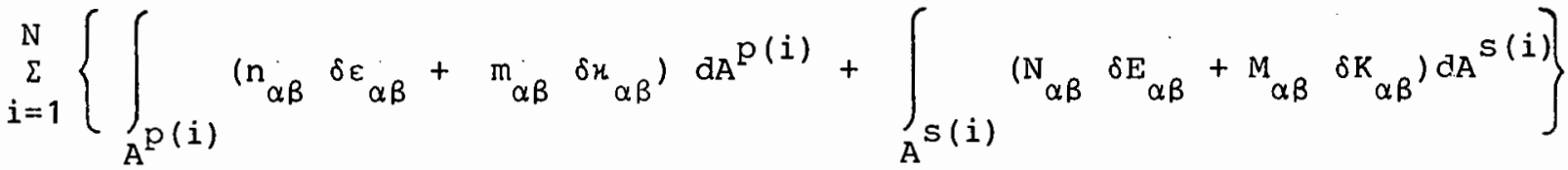

$$
\begin{aligned}
& =\delta w_{\text {ext }}^{p}+\delta w_{\text {ext }}^{s}
\end{aligned}
$$

where $A^{p}$ and $A^{S}$ are the areas of the $i^{\text {th }}$ panel and stiffener respectively.

By substitution of the strain-displacement relations (3.7) to (3.10) for the variational quantities in the above equation and by using Green's theorem (Malvern (1969), pp.197-200) we can obtain the equilibriun equations and the corresponding boundary conditions as shown in Appendix 3B.

To find the expressions for external virtual work we proceed as follows: the cylinder is assumed to be simply supported at its ends with a compressive traction of magnitude $\sigma$ acting on the end areas $A_{e}^{p(i)}$ and $A_{e}^{S(i)}$ of the $i^{\text {th }}$ panel and stiffener respectively. Therefore the external virtual work is given by

$$
\begin{aligned}
& \delta w_{e x t}^{p}=\sum_{i=1}^{N} \int_{A} \int_{e} r \sigma\left(\left.\delta u^{*}\right|_{x=0}-\left.\delta u^{*}\right|_{x=\ell}\right) d A_{e}^{p(i)} \\
& \delta w_{e x t}^{S}=\sum_{i=1}^{N} \int_{A}^{S(i)} \int_{e} r \sigma\left(\left.\delta u^{*}\right|_{x=0}-\left.\delta U^{*}\right|_{x=\ell}\right) d A_{e}^{S(i)}
\end{aligned}
$$

where $\delta u^{*}$ and $\delta U^{*}$ are the virtual displacements in the $\mathrm{x}$ direction at the ends of the $i^{\text {th }}$ panel and stiffener. Since the compressive traction is constant over the whole area at the ends equations (3.34) become

$$
\delta W_{e x t}^{p}=\sigma r \sum_{i=1}^{N} \int_{A}^{p(i)}\left[\delta u^{*}(0)-\delta u^{*}(\ell)\right] d A_{e}^{p(i)}=\sigma^{N} \sum_{i=1} \int_{A} e_{e}(i) \int_{\ell} \frac{\partial}{\partial x}\left(\delta u^{*}\right) d A_{e}^{p(i)} d x
$$




$$
\delta W_{\text {ext }}^{S}=\sigma r \sum_{i=1}^{N} \int_{A_{e}(i)}\left[\delta U^{*}(0)-\delta U^{*}(l)\right] d A_{e} s(i)=\sigma \sum_{i=1}^{N} \int_{A} s(i) \int_{l} \frac{\partial}{\partial x}\left(\delta U^{*}\right) d A_{e} S(i) d x
$$

Due to the ends of the cylinder being simply-supported the displacement across the thickness of the $i^{\text {th }}$ panel can be given by

$$
u^{*(i)}=\left.\left(u^{(i)}-z w^{(i)}\right)\right|_{x=0, \ell}
$$

For the stiffeners there are no rotations of the areas at the ends of the cylinder in the $y$ direction, but there are in the $z$ direction, so that for the $i^{\text {th }}$ stiffener,

$$
U^{*(i)}=\left.\left(U^{(i)}-z w_{, x}^{(i)}\right)\right|_{x=0, \ell}
$$

thus, $\quad \delta u^{*(i)}=\left(\delta u^{(i)}-\left.z \delta w_{, x}^{(i)}\right|_{x=0, l}\right.$

$$
\delta U^{*(i)}=\left.\left(\delta U^{(i)}-z \delta W^{(i)}\right)\right|_{x=0, \ell}
$$

Substitution of equations (3.36) into equations (3.35) and integrating through the thicknesses of the panels and stiffeners results in the following external virtual work equations for the $i^{\text {th }}$ panel and stiffener:

$$
\begin{aligned}
& \delta w_{e x t}^{p(i)}=-\left.\sigma t_{p} \int_{A} \int_{p(i)} \delta u_{, x}^{(i)}\right|_{x=0, \ell} d A^{p(i)}, \\
& \delta w_{e x t}^{S(i)}=-\left.\sigma t_{S} r \int_{A} \int_{S(i)} \delta U_{x}^{(i)}\right|_{x=0, \ell} d A^{s(i)} ;
\end{aligned}
$$

here $A^{p(i)}$ and $A^{S(i)}$ are the midsurface areas of the $i^{\text {th }}$ panel and stiffener respectively. 


\subsection{Assumed Prebuckling Mode}

\subsubsection{Assumed Displacement Functions}

The shape of the initial imperfections must first be extablished since they determine the choice of the displacement functions. The initial imperfection chosen is that which corresponds approximately to the shape of the buckling mode of a perfect stiffened cylinder (Syngellakis and Walker (1978b), Reddy (1980)); that is, the radial imperfection in the shell consists of a sinusoidal variation in the axial direction, the number of half-waves being left arbitrary, while the circumferential variation is a single half sine wave between two stiffeners, symmetrical about the lines $\theta^{(i)}=0$ (see Fig. 3.2). Thus,

$$
\bar{w}^{(i)}=\mu^{(i)} \sin \alpha \xi \cos \beta \theta^{(i)}
$$

with

$$
\mu^{(i+1)}=-\mu^{(i)}
$$

where $\mu^{(i)}$ is the imperfection amplitude in the $i^{\text {th }}$ panel, $\xi=x / r$, $\alpha=m \pi r / \ell$ where $m$ is the number of axial half-waves, and $\beta=N / 2$. Furthermore, the corresponding imperfection in the $i^{\text {th }}$ stiffener can be obtained from the last of equations (3.6), i.e.

$$
\bar{W}^{(i)}=\frac{\beta}{r}(y-e) \mu^{(i)} \sin \alpha \xi .
$$

Note that

$$
\vec{w}^{(i)}\left(x, \theta_{0}\right)=\bar{w}^{(i)}(x, e)=\vec{w}_{, x}^{(i)}\left(x, \theta_{0}\right)=0
$$

but

$$
\bar{W}^{(i)}(x, y)=-\frac{1}{r}(y-e) \bar{w}_{, \theta}^{(i)}\left(x, \theta_{0}\right)
$$

Now the displacement functions are chosen so that the following symmetry conditions (Syngellakis and Walker (1978a)) for the panels are satisfied:

$$
\begin{aligned}
& u^{(i)}(x, \theta)=u^{(i)}(x,-\theta), \\
& v^{(i)}(x, \theta)=-v^{(i)}(x,-\theta) \\
& w^{(i)}(x, \theta)=w^{(i)}(x,-\theta) .
\end{aligned}
$$




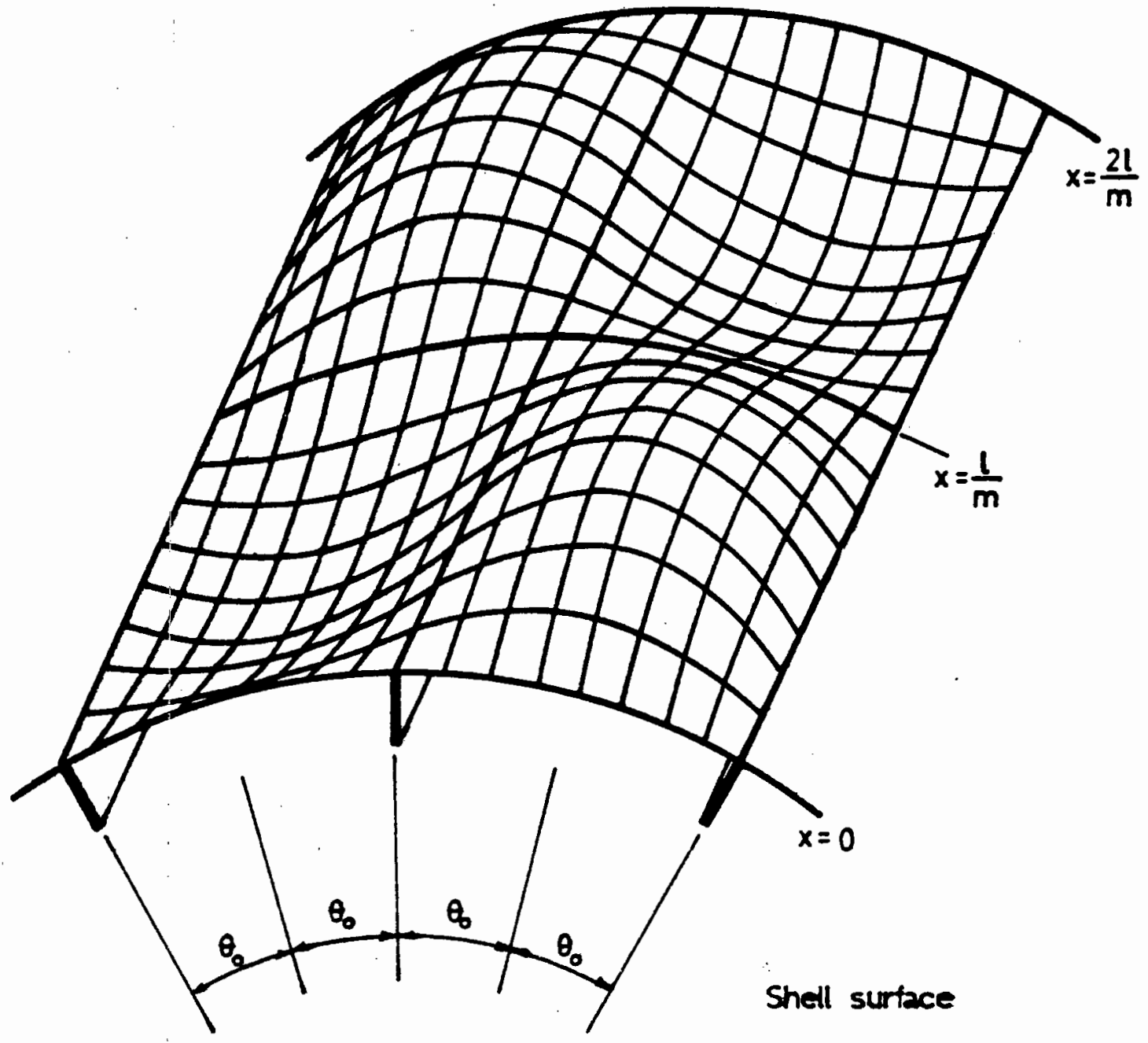

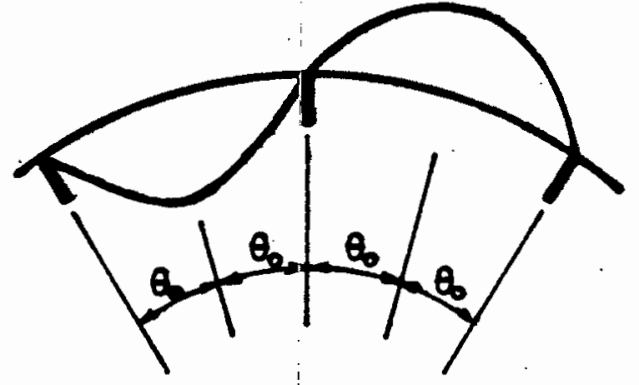

Section through $x=1 / 2 m$

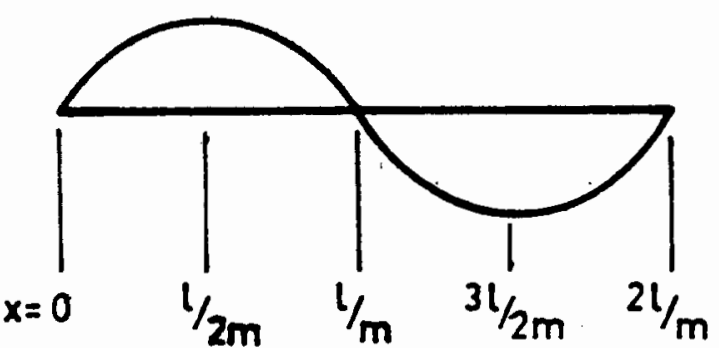

Section through panel mid-generater

Fig.3.2 Variation of Imperfection over Surface (showing a full wave in each direction) 
The radial displacement $w^{(i)}$ must comprise a uniform expansion and a term of the same shape as the initial imperfection which would allow for a "growth" of this imperfection. The symmetry of $w^{(i)}$ and $\bar{w}^{(i)}$ is supported by experimental evidence of Walker and Davies (1976). By considering the boundary conditions as shown in Appendix 3B we obtain the other displacement functions. Thus the displacement functions for the $i^{\text {th }}$ panel are

$$
\begin{aligned}
& u^{(i)}=u_{0_{1}}^{(i)} \xi+u_{0_{2}}^{(i)} \cos \alpha \xi \cos \beta \theta^{(i)} \\
& v^{(i)}=v_{0_{1}}^{(i)} \sin \alpha \xi \sin \beta \theta^{(i)} \\
& w^{(i)}=w_{0_{1}}^{(i)}+w_{0_{2}}^{(i)} \sin \alpha \xi \cos \beta \theta^{(i)}
\end{aligned}
$$

with $\quad u_{01}^{(i+1)}=u_{01}^{(i)}$

$$
\begin{aligned}
& u_{0_{2}}^{(i+1)}=-u_{0_{2}}^{(i)} \\
& v_{0_{1}}^{(i+1)}=-v_{0_{1}}^{(i)} \\
& w_{0_{1}}^{(i+1)}=w_{0_{1}}^{(i)} \\
& w_{0_{2}}^{(i+1)}=-w_{0_{2}}^{(i)}
\end{aligned}
$$

Using equations (3.6) the displacement functions for the $i^{\text {th }}$ stiffener are obtained. These are

$$
\begin{aligned}
& U^{(i)}=u_{01}^{(i)} \xi \\
& V_{i}^{(i)}=\zeta w_{0_{1}}^{(i)} \\
& W^{(i)}=\left[\underset{O_{1}}{\left[v^{(i)}\right.}+\frac{\beta}{r}(y-e) \underset{o_{1}}{(i)}\right] \sin \alpha \xi
\end{aligned}
$$

where $\quad \zeta=1+\frac{1}{r}(y-e)$. This factor is derived in Appendix $3 c$.

The displacement functions in equations (3.43) and (3.45) are the same as those used by syngellakis and walker (1978b). The displacement functions for the stiffeners obey the boundary conditions obtained from the principal of virtual work; these are given in Appendix $3 \mathrm{~B}$. 


\subsubsection{Complete Strains at a Point}

For the $i^{\text {th }}$ panel the midsurface membrane and bending strains are obtained by substituting equations (3.38) and (3.43) into equations (3.7) and (3.8) . For the $i^{\text {th }}$ stiffener the midsurface membrane and bending strains are likewise obtained by substituting equations (3.40) and (3.45) into equations (3.9)' and (3.10). These panel and stiffener strains are shown in Appendix 3D. By using equations (3.'11) and (3.12), we can obtain the strains at any point in the $i^{\text {th }}$ panel and stiffener. Thus, the expressions for the strains in the $i^{\text {th }}$ panel and stiffener, involve the five displacement parameters $u_{0_{1}}^{(i)} u_{0_{2}}^{(i)}$, $v_{0_{1}}^{(i)}, w_{0_{1}}^{(i)}, w_{0_{2}}^{(i)}$ and the imperfection amplitude $\mu^{(i)}$.

For any particular problem $\mu^{(i)}$ is fixed and the strain tensors are symmetric (see equations (3.7) to (3.10)) so that for a typical panel and stiffener the strain-displacement relations may be expressed in the form

$$
\begin{aligned}
& \underline{n}=\underline{B}^{\mathrm{p}} \underline{\mathrm{u}} . \\
& \underline{\mathrm{H}}=\underline{\mathrm{B}} \underline{\mathrm{s}} \underline{\mathrm{u}}
\end{aligned}
$$

where

$$
\begin{aligned}
& \underline{\eta}^{T}=\left(n_{x x}, n_{\theta \theta}, n_{x \theta}\right), \\
& \underline{H}^{T}=\left(H_{x x}, H_{y y}, H_{x y}\right), \\
& \underline{u}^{T}=\left(u_{01}, u_{0_{2}}, v_{01}, w_{0_{1}}, w_{02}\right)
\end{aligned}
$$

and 


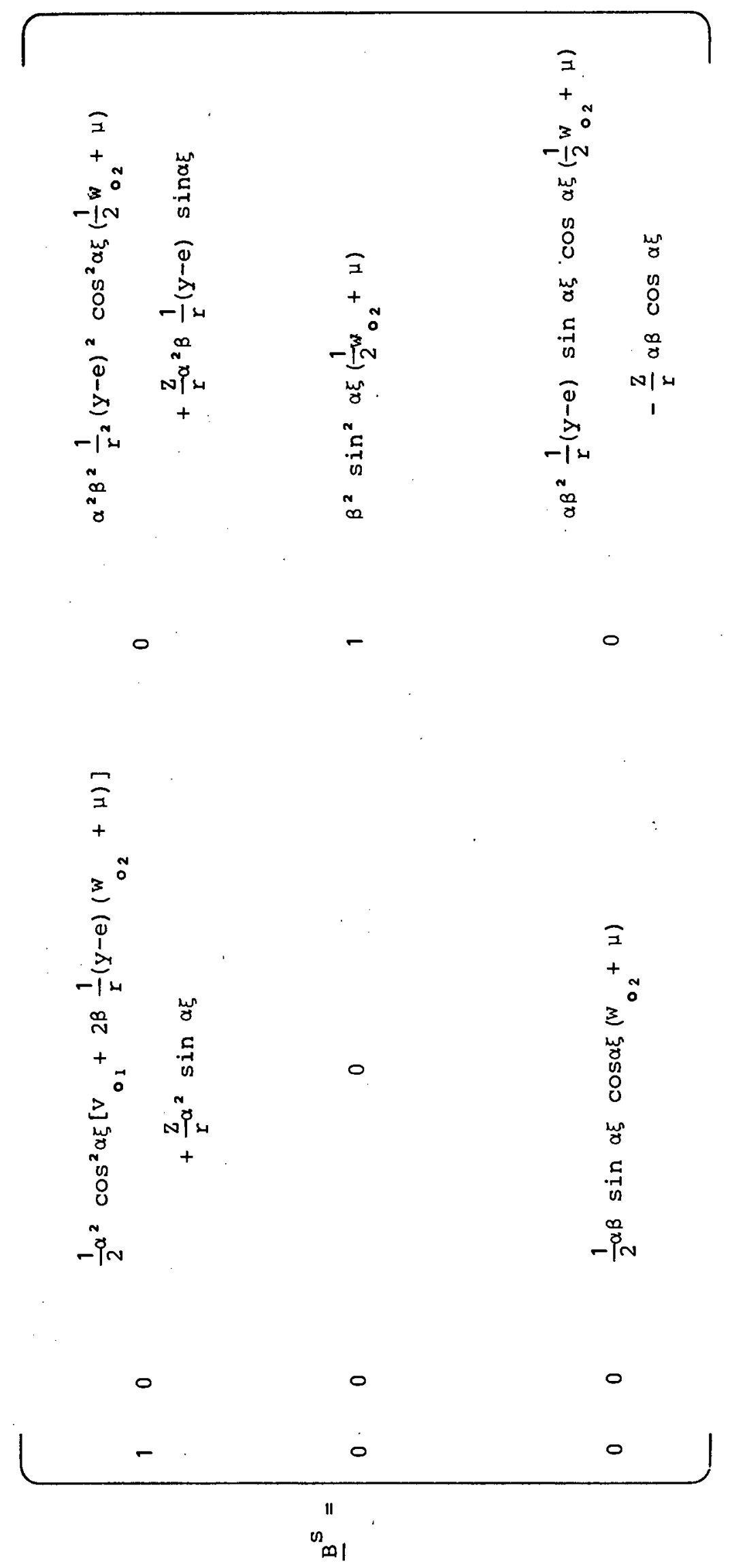




\subsubsection{Virtual Work Equations}

In the present analysis the virtual work equation (3.33) is used directly, with variations in the displacements reducing to variations in the displacement parameters. Thus, for the $i^{\text {th }}$ panel,

$$
\begin{aligned}
& \delta u^{(i)}=\delta u_{0_{1}}^{(i)} \xi+\delta u_{0_{2}}^{(i)} \cos \alpha \xi \cos \beta \theta^{(i)}, \\
& \delta v^{(i)}=\delta v_{0_{1}}^{(i)} \sin \alpha \xi \sin \beta \theta^{(i)}, \\
& \delta w^{(i)}=\delta w_{0_{1}}^{(i)}+\delta w_{0_{2}}^{(i)} \sin \alpha \xi \cos \beta \theta^{(i)},
\end{aligned}
$$

and for the $i^{\text {th }}$ stiffener,

$$
\begin{aligned}
\delta U^{(i)} & =\delta u_{0_{1}}^{(i)} \xi \\
\delta v^{(i)} & =\delta \delta w_{0_{1}}^{(i)} \\
\delta W^{(i)} & =\left[\delta v_{0_{1}}^{(i)}+\frac{B}{r}(y-e) \delta w_{0_{2}}^{(i)}\right] \sin \alpha \xi .
\end{aligned}
$$

The external virtual work equations (3.37) can be written in terms of the displacement parameters by using equations (3.48) and (3.49) which, after the integrations have been carried out, result in

$$
\left(\delta w_{\text {ext }}^{p}+\delta w_{\text {ext }}^{s}\right)^{(i)}=2 \sigma r \ell\left(t_{p} \theta_{0}+\frac{t_{s} d}{r}\right) \delta u_{o_{1}}^{(i)}
$$

for the $i^{\text {th }}$ panel plus $i^{\text {th }}$ stiffener.

Substitution of the strain-displacement relations (3.7) to (3.10) into the left hand side of equation (3.33), using (3.48) and (3.49), and substitution of equation (3.50) into the right hand side of equation (3.33), leads to the virtual work formulation shown in Appendix 3D. For an elastic material this formulation reduces to that obtained by Syngellakis and Walker (1978b).

The principal of virtual work (3.33) may be expressed in the form

$$
\sum_{i=1}^{N}\left[\delta \underline{u}^{(i)}\left\{\iint_{A} p(i) \underline{J}^{p} \underline{r} d A^{p(i)}+\int_{A} s(i) \underline{J}^{S} \underline{R}_{d A} s(i)\right\}-\delta \underline{u}^{(i)} \underline{F}^{(i)}\right]=0
$$


where

$$
\begin{aligned}
& \underline{u}^{(i)}=\left(\delta u_{o_{1}}^{(i)}, \delta u_{o_{2}}^{(i)}, \delta o_{1}^{(i)},{ }_{o_{1}}^{\delta w_{1}^{(i)}}, \delta o_{2}^{(i)}\right)^{T}, \\
& \underline{F}^{(i)}=\left(-2 \sigma r \ell\left[t_{p} \theta_{0}+\frac{t_{s} d}{r}\right], 0,0,0,0\right)^{T}
\end{aligned}
$$

and 


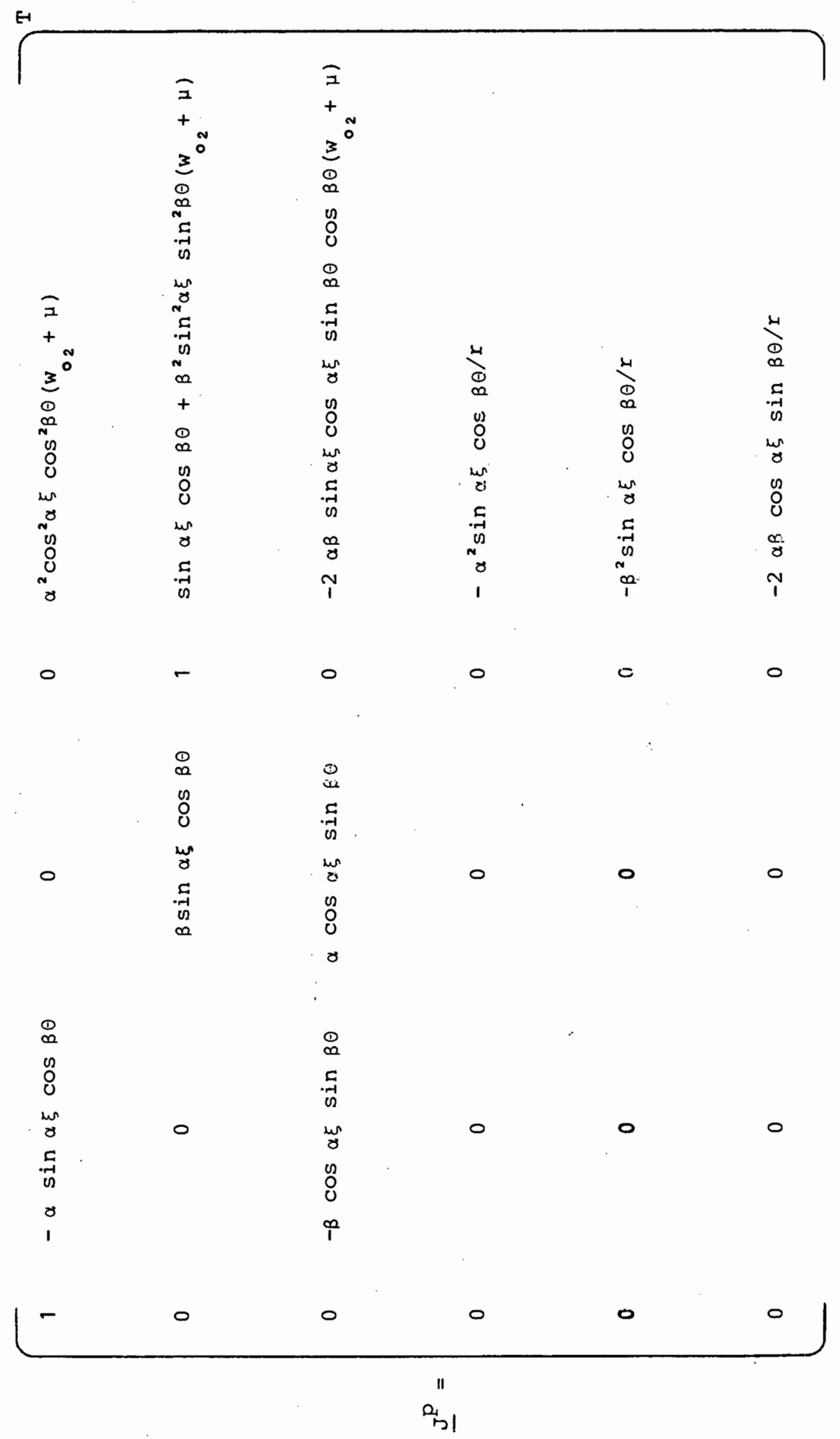




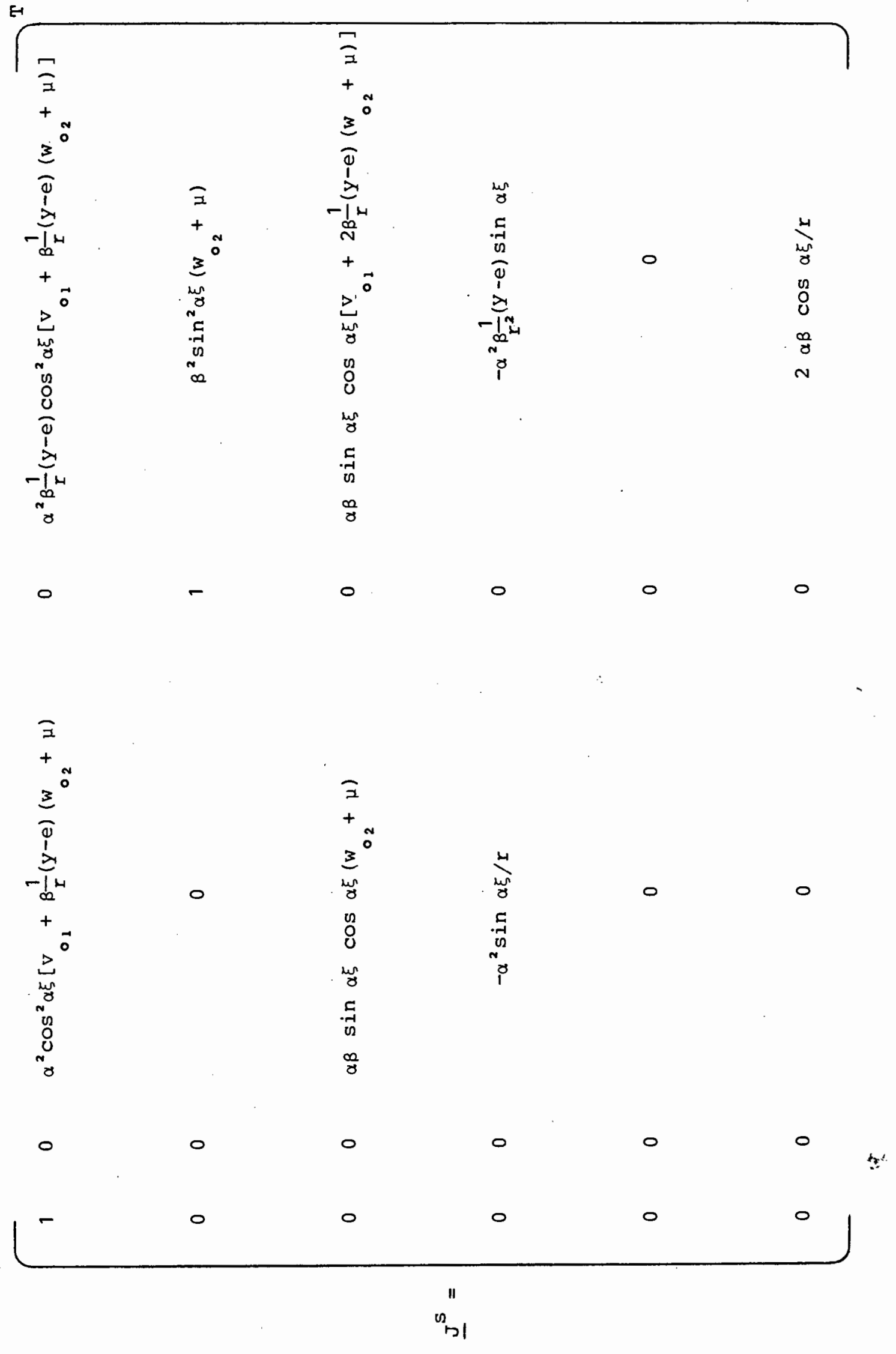




\subsection{Concluding. Remarks}

By combining all the matrix equations in this chapter we have the complete matrix formulation. Thus, by using equations $(3.25),(2.26),(3.29)$, $(3.30),(3.30),(3.46),(3.47)$ and $(3.51)$ we obtain

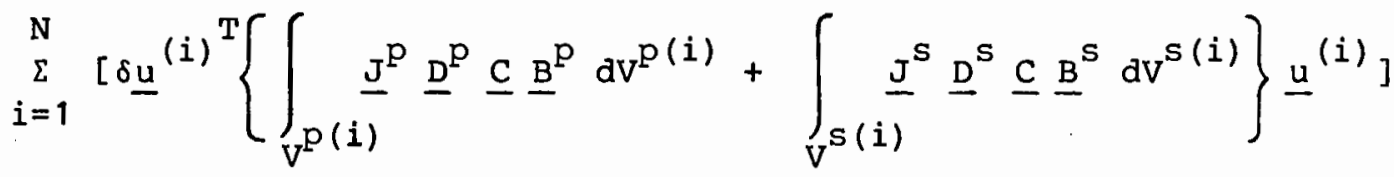

$$
\begin{aligned}
& -\sum_{i=1}^{N}\left[\delta \underline{u}^{(i)^{T}} \underline{F}^{(i)}\right]=0
\end{aligned}
$$

where the matrices $\underline{J}, \underline{D}, \underline{C}$ and $\underline{B}$ are all functions of position and have been defined for a typical panel and a typical stiffener in this chapter.

It is now possible to obtain complete information about the primary path for a stiffened cylinder subjected to an axial compressive load. The geometry of the stiffened cylinder, the initial imperfection as well as the material behaviour are arbitrary within the assumptions and theories used in this chapter. The numerical procedures used in obtaining these results are discussed in the following chapter. The question of bifurcation from the primary path is then addressed in chapter 5 . 
APPENDIX 3A Plane stress constitutive coefficients $C_{\alpha \beta \gamma \gamma}$

The three dimensional constitutive equation is as follows

$$
\sigma_{i j}=\bar{C}_{i j k \ell} \varepsilon_{k \ell}
$$

where all indices range from 1 to 3 , but for plane stress the equation is

$$
\sigma_{\alpha \beta}=c_{\alpha \beta \kappa \gamma} \varepsilon_{\kappa \gamma}
$$

where all indices range from 1 to 2 . The following relation will now be derived

$$
c_{\alpha \beta \varkappa \gamma}=\bar{c}_{\alpha \beta \mu \gamma}-\frac{\bar{C}_{\alpha \beta 33} \bar{c}_{33 \kappa \gamma}}{\bar{C}_{3333}}
$$

For a state of approximate plane stress in the shell, we assume that

$$
\sigma_{13}=\sigma_{31}=\sigma_{23}=\sigma_{32}=\sigma_{33}=0 \text {. }
$$

It follows that

$$
\varepsilon_{13}=\varepsilon_{31}=\varepsilon_{23}=\varepsilon_{32}=0 \text { but } \varepsilon_{33} \neq 0
$$

Equation (A.1) then becomes

$$
\begin{aligned}
& \sigma_{11}=\bar{C}_{1111} \varepsilon_{11}+\bar{C}_{1122} \varepsilon_{22}+\bar{C}_{1133} \varepsilon_{33} \\
& \sigma_{22}=\bar{C}_{2212} \varepsilon_{11}+\bar{C}_{2222} \varepsilon_{22}+\bar{C}_{2233} \varepsilon_{33} \\
& \sigma_{33}=0=\bar{C}_{3311} \varepsilon_{11}+\bar{C}_{3322} \varepsilon_{22} \bar{C}_{3333} \varepsilon_{33} \\
& \sigma_{12}=\bar{C}_{1212} \varepsilon_{12} \\
& \sigma_{21}=\bar{C}_{2121} \varepsilon_{21}
\end{aligned}
$$

with all the other coefficients of $\bar{c}_{i j k \ell}=0$. 
But using equation (A.2) we obtain

$$
\begin{aligned}
& \sigma_{1 i}=C_{1111} \varepsilon_{12}+C_{1122} \varepsilon_{22} \\
& \sigma_{22}=C_{2211} \varepsilon_{11}+C_{2222} \varepsilon_{22} \\
& \sigma_{12}=C_{1212} E_{12}
\end{aligned}
$$

$$
\sigma_{21}=C_{2121} \varepsilon_{21}
$$

By using the third of equations (A.4) we can obtain $\varepsilon_{33}$ which can be substituted into the first two equations (A.4). Then by equating the relevant equations of $(A .4)$ and $(A .5)$ it is easily shown that the relation (A.3) holds. 
APPENDIX 3B Equilibrium equations and boundary conditions.

The governing equations obtained from the use of Green's theorem in the variational equation (3.33) are:

(a) For any panel

$$
\begin{aligned}
& n_{x x, x}+\frac{1}{r} n_{x \Theta, \theta}=0 \\
& n_{x \Theta, x}+\frac{1}{r} n_{\theta \theta, \theta}=0 \\
& m_{x x, x x}+\frac{2}{r} m_{x \theta, x \theta}+\frac{1}{r^{2}} m_{\theta \theta, \theta \theta}+\frac{1}{r} n_{\theta \theta}-n_{x x}\left(w_{, x x}+\bar{w}_{, x x}\right) \\
& \quad-\frac{2}{r} n_{x \theta}(w, x \theta \\
& \left.+\bar{w}_{, x \theta}\right)-\frac{1}{r^{2}} n_{\theta \theta}(w, \theta \theta+\bar{w}, \theta \theta)=0
\end{aligned}
$$

(b) For any stiffener

$$
\begin{aligned}
& \mathrm{N}_{x x, x}+\mathrm{N}_{x y, y}=0 \\
& \mathrm{~N}_{x y, x}+\mathrm{N}_{Y y, y}=0 \\
& M_{x x, x x}+2 M_{x y, x y}+M_{y y, y y}-N_{x x}\left(w, x x+\bar{w}_{, x x}\right) \\
& -2 \mathrm{~N}_{x y}\left(\mathrm{w}_{, X Y}+\overline{\mathrm{W}}_{, x y}\right)-\mathrm{N}_{Y Y}\left(\mathrm{~W}_{, Y Y}+\overline{\mathrm{W}}_{, Y Y}\right)=0
\end{aligned}
$$


The boundary conditions are:

(c) For any panel: at $\mathrm{x}=0, \ell$

$$
\begin{aligned}
& n_{x x}-\sigma t_{p}=0 \\
& \text { or } \quad \delta u=0 \\
& \mathrm{n}_{\mathrm{x} \theta}=0 \\
& \text { or } \quad \delta v=0 \\
& m_{x x}=0 \\
& \text { or } \quad \delta w, x=0 \\
& m_{x x, x}+\frac{2}{r} m_{x \theta, \theta}-n_{x x}\left(w, x+\bar{w}_{, x}\right) \\
& -\frac{1}{r} n_{x \Theta}\left(w, \theta+\bar{w}_{, \theta}\right)=0 \quad \text { or } \quad \delta w=0 \\
& N_{x x}-\sigma t_{s}=0 \quad \text { or } \quad \delta U=0 \\
& \mathrm{~N}_{\mathrm{xy}}=0 \quad \text { or } \quad \delta \mathrm{V}=0 \\
& M_{\mathrm{xx}}=0 \quad \text { or } \quad \delta W_{, \mathrm{x}}=0 \\
& M_{x x, x}+2 M_{x y, y}-N_{x x}\left(W_{, x}+\bar{W}, x\right) \\
& -\mathrm{N}_{x y}\left(\mathrm{~W}, \mathrm{y}+\overline{\mathrm{W}}, \mathrm{y}^{\prime}=0 \quad \text { or } \quad \delta w=0\right. \\
& \text { at } y=+d,-d \\
& \begin{array}{lll}
\mathrm{N}_{\mathrm{XY}}=0 & \text { or } & \delta \mathrm{U}=0 \\
\mathrm{~N}_{Y Y}=0 & \text { or } & \delta \mathrm{V}=0
\end{array}
\end{aligned}
$$




$$
\begin{array}{ccc}
M_{Y Y}=0 & \text { or } & \delta W_{, Y}=0 \\
M_{Y Y, Y}+{ }^{2} M_{X Y, X}-N_{Y Y}\left(W_{, Y}+\bar{W}_{, Y}\right) & \\
-N_{X Y}\left(W_{, X}+\bar{W}_{, X}\right)=0 \quad \text { or } & \delta W=0
\end{array}
$$


APPENDIX 3C Derivation of expression for stiffener displacement $V^{(i)}(x, y)$

Consider a perfect stiffeneed cylinder and assume that $\mathrm{V}$ is a linear function of $y$ :

$$
\mathrm{V}=\mathrm{ay}+\mathrm{b}
$$

where $\mathrm{a}$ and $\mathrm{b}$ are constants to be determined by the boundary conditions. From the boundary conditions in Appendix $3 \mathrm{~B}$ which are similar to those of a perfect shell, $\mathrm{N}_{\mathrm{YY}}=0$ at $\mathrm{y}=\mathrm{d}$. Thus

$$
0=\sigma_{Y Y}=\frac{E_{S}}{\left(1-v_{S}^{2}\right)}\left[\varepsilon_{X}+v_{S} \varepsilon_{Y}\right]
$$

where $\varepsilon_{\mathrm{x}}$ and $\varepsilon_{\mathrm{y}}$ are the strains. Therefore

$$
\varepsilon_{x}=-v_{s^{\prime}} \varepsilon_{y} ;
$$

but, from equations (3.9) and (3.45), for the perfect cylinder we have

$$
\varepsilon_{x}=u_{01} / r
$$

and

$$
w_{0_{1}}=-v_{S_{1}} u_{0_{1}}
$$

These equations imply $\varepsilon_{y}=w_{0_{1}} / r$

Now from equation (C.1) and (3.9)

$$
\varepsilon_{y}=\frac{\partial V}{\partial y}=a
$$

Thus

$$
a=w_{01} / r
$$

Another condition to be satisfied is in equations (3.5) where we find that $V=w_{0_{1}}$ at $y=e . \quad$ Thus, using equation $(C .1)$ and substituion, we obtain $w / \cdots \cdots$ 
38

$$
w_{0_{1}}=\frac{w_{0_{1}}}{r} e+b .
$$

Therefore $\quad b=w_{0_{1}}\left(1-\frac{e}{r}\right)$

(C. 3)

and equation (C.1) becomes

$$
V=w_{01}\left[1+\frac{1}{r}(y-e)\right]
$$

or

$$
V=\zeta \mathrm{w}_{0_{1}}
$$

where

$$
\zeta=1+\frac{1}{r}(y-e) .
$$


APPENDIX 3D Strain and Virtual Work equations.

Membrane and bending strains for a typical panel:

(superscripts (i) are omitted for clarity)

$$
\begin{aligned}
& \varepsilon_{x x}=u_{o_{1}}-\alpha u_{o_{2}} \sin \alpha \xi \cos \beta \theta+\alpha^{2} \cos ^{2} \alpha \xi \cos ^{2} \beta \theta\left(\frac{1}{2} w_{o_{2}}^{2}+\mu w_{o_{2}}\right) \\
& \varepsilon_{\theta \theta}=\sin \alpha \xi \cos \beta \theta\left(\beta v_{0_{1}}+w_{0_{2}}\right)+w_{0_{1}}+\beta^{2} \sin ^{2} \alpha \xi \sin ^{2} \beta \theta\left(\frac{1}{2} w_{0_{2}}^{2}+\mu_{w_{0}}\right) \\
& \varepsilon_{x \theta}=\frac{1}{2} \cos \alpha \xi \sin \beta \theta\left(\alpha v_{O_{1}}-\beta u_{O_{2}}\right)-\alpha \beta \sin \alpha \xi \cos \alpha \xi \sin \beta \theta \cos \beta \theta\left(\frac{1}{2} w_{O_{2}}^{2}+\mu_{O_{2}}\right) \\
& n_{x x}=\frac{1}{\mathbf{r}_{02}} \alpha^{2} \sin \alpha \xi \cos \beta \theta \\
& u_{\theta \theta}=-\frac{1}{r_{0}} \beta^{2} \sin \alpha \xi \cos \beta \theta \\
& \varkappa_{x \Theta}=-\frac{1}{\mathbf{r}_{02}} w_{0 \beta} \cos \alpha \xi \sin \beta \theta
\end{aligned}
$$

Membrane and bending strains for a typicial stiffener:

$$
\begin{aligned}
& E_{x X}=u_{0_{1}}+\frac{1}{2} \alpha^{2} \cos ^{2} \alpha \xi\left[v_{O_{1}}^{2}+2 \beta \frac{1}{r}(y-e) v_{O_{1}}\left(w_{O_{2}}+\mu\right)+\frac{1}{r^{2}}(y-e)^{2} \beta^{2}\left(w_{O_{2}}^{2}+2 w_{O_{2}}^{\mu} \mu\right]\right. \\
& E_{Y Y}=w_{O_{1}}+\beta^{2} \sin ^{2} \alpha \xi\left(\frac{1}{2} w_{O_{2}}^{2}+\mu w_{O_{2}}\right) \\
& E_{x y}=\frac{1}{2} \alpha \beta \sin \alpha \cdot \cos \alpha \xi\left[v_{0_{1}}\left(w_{0_{2}}+\mu\right)+\beta \frac{1}{r}(y-e)\left(w_{0_{2}}^{2}+2 w_{0_{2}} \mu\right)\right] \\
& k_{x x}=\frac{1}{r} \alpha^{2} \sin \alpha \xi\left[v_{0_{1}}+\beta \frac{1}{r}(y-e) w_{0_{2}}\right] \\
& K_{y y}=0 \\
& \mathrm{~K}_{\mathrm{xy}}=\frac{1}{\mathrm{r}_{02}} \alpha \beta \cos \alpha \xi
\end{aligned}
$$


The virtual work equation is as follows:

$$
\begin{aligned}
& \sum_{i=1}^{N}\left[\left\{\int_{A(i)} n_{x x} d A^{p(i)}+\int_{A} s(i){ }_{x x} d A^{s(i)}+2 \sigma r l\left(t_{p} \theta_{0}+\frac{t_{s} d}{r}\right)\right\} \delta u_{0_{1}}^{(i)}+\right.
\end{aligned}
$$

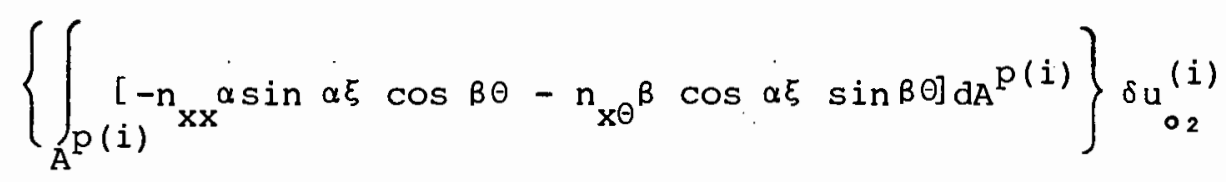

$$
\begin{aligned}
& \left\{\int_{A}\left[n_{x} n_{x} \alpha \cos \alpha \xi \sin \beta \Theta+n_{\theta \Theta} \beta \sin \alpha \xi \cos \beta \theta\right] d A(i)+\int_{A}\left[(i) x^{\alpha^{2} v_{0}^{(i)}} \cos ^{2} \alpha \xi\right.\right.
\end{aligned}
$$$$
+\mathrm{N}_{x y} \alpha \beta \cos \alpha \xi \sin \alpha \xi\left(w_{0_{2}}+\mu\right)^{(i)}+N_{x x} \alpha^{2} \beta \frac{1}{r}(y-e) \cos ^{2} \alpha \xi\left(w_{o_{2}}+\mu\right)(i)
$$$$
\left.\left.-M_{x x} \frac{1}{r} \alpha^{2} \sin \alpha \xi\right] d A s(i)\right\} \delta v_{0_{1}}^{(i)}+
$$$$
\left\{\int_{A} p(i) n_{\Theta \Theta} d A^{p(i)}+\int_{A} s(i){ }_{Y Y}^{N} d A s(i)\right\} \delta w_{O_{1}}^{(i)}+
$$$$
\left\{\int _ { A } \left[( i ) \left[n_{x x} \alpha^{2} \cos ^{2} \alpha \xi \cos ^{2} \beta \theta\left(w_{o_{2}}+\mu\right){ }^{(i)}\right.\right.\right.
$$

$\left.-2 n_{x} \alpha \beta \sin \alpha \xi \cos \alpha \xi \sin \beta \theta \cos \beta \theta_{\left(w_{02}\right.}+\mu\right)^{(i)}+n_{\theta \theta} \sin \alpha \xi \cos \beta \theta$

$+n_{\theta \theta^{\beta}} \sin ^{2} \alpha \xi \sin ^{2} \beta \theta\left(w_{o_{2}}+\mu\right)^{(i)}-m_{x x} \frac{1}{r} \alpha^{2} \sin \alpha \xi \cos \beta \theta-m_{x \theta} \frac{2}{r} \alpha \beta \cos \alpha \xi \sin \beta \theta$ 
41

$$
\begin{aligned}
& \left.-m_{\theta \Theta} \frac{1}{r^{2}} \sin \alpha \xi \cos \beta \theta\right] d A^{p(i)}+\int_{A}\left[N_{x x} \alpha^{2} B \frac{1}{r}(y-e) v_{O_{1}}^{(i)} \cos ^{2} \alpha \xi\right. \\
& { }_{x x}^{+N} \alpha^{2} \beta^{2} \frac{1}{r^{2}}(Y-e)^{2} \cos ^{2} \alpha \xi\left(w{ }_{02}+\mu\right)(i) \\
& \mathrm{N}_{x y} \alpha \beta \cos \alpha \xi \sin \alpha \xi\left(v_{0_{1}}^{(i)}+2 \beta \frac{1}{\mathrm{r}}(y-e)\left(w_{0_{2}}+\mu\right)(i),\right. \\
& +N_{Y Y} \beta^{2} \sin ^{2} \alpha \xi\left(w_{O 2}+\mu\right)^{(i)}-M_{X x} \alpha^{2} \beta \frac{1}{r^{2}}(Y-e) \sin \alpha \xi \\
& \left.\left.\left.+2 M_{x y} \frac{1}{r} \alpha \beta \cos \alpha \xi\right] d A(i)\right\} \delta w_{O_{2}}^{(i)}\right]=0
\end{aligned}
$$




\section{CHAPTER $\quad 4$ \\ NUMERICAL PROCEDURES FOR THE PRIMARY EQUILIBRIUM PATH}

\subsection{Introductory Remarks}

From the virtual work formulation described in Chapter 3 we are able to obtain five non-linear equations in the displacement parameters. The solution of these equations provides complete information about the primary equilibrium path. The numerical procedures used to solve the non-linear equations are described in section 4.2. For this analysis numerical integrations over the midsurface areas, in order to obtain the internal virtual work, and through the thicknesses, in order to obtain the stress resultants, for both the stiffeners and panels, are required; these formulae are described in Section 4.3. To determine the correct constitutive coefficients in the plastic range an iterative procedure described in section 4.4 is required and thus the stresses at any point in the shell can be determined.

\subsection{Primary path solutions}

\subsubsection{Modified Newton-Raphson Iteration}

In equation (3.51) the variations of the five displacement parameters are arbitrary so that, by setting their coefficients equal to zero, we obtain five non-linear equations in the displacement parameters which can be written as

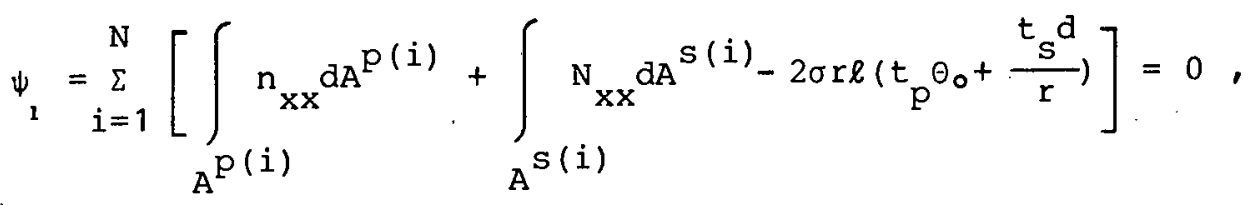

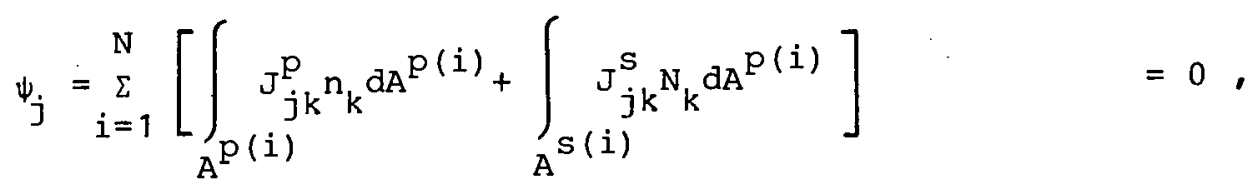

$$
\begin{aligned}
& j=\Lambda, \ldots, 4 \text {, }
\end{aligned}
$$

since the vector $E$ has only its first term non-zero. Here, as in equation (3.52), for a typical panel and stiffener we have 


$$
\begin{aligned}
n_{k}= & \int_{-t_{p / 2}}^{t_{p} / 2} D_{k \ell}^{p} c_{\ell m} n_{m} d z, \\
N_{k}= & \int_{-t_{s} / 2}^{t_{s}^{s}} D_{k \ell}^{s} c_{\ell m} H_{m} d z .
\end{aligned}
$$

Here $\mathrm{k}=1, \ldots, 6$ and $\ell, \mathrm{m}=1,2,3$.

The displacement parameter $u$, corresponding to the end shortening of the cylinder is chosen as the loading parameter. Thus for a specific value of $u_{0_{1}}$ the other displacement parameters $\left(u_{0_{2}}, v_{0_{1}}, w_{O_{1}}, w_{0_{2}}\right)$ can be solved from equations (4.2) using Newton-Raphson iteration and then the external axial stress. $\sigma$, can be obtained directly from equation (4.1).

The Newton-Raphson iteration process can be formulated as follows: with $\mathrm{n}$ ranging from 1 to 4 a truncated Taylor series expansion about the solution $t_{u_{n}}$ at the $t^{\text {th }}$ iteration gives

$$
t_{\psi_{j}}={ }^{t-1} \psi_{j}+\left.\frac{\partial \psi}{\partial u_{n}}\right|_{t-1}\left(u_{n} u_{n}-t-1 u_{n}\right),
$$

where higher terms are neglected. From equations (4.2) we require that

$$
\begin{aligned}
& { }^{t} \psi_{j}=0, \text { so that if we write } \\
& \left.\frac{\partial \psi_{j}}{\partial u_{n}}\right|_{t-1 u_{n}}={ }^{t-1} Q_{j n}
\end{aligned}
$$

and

$$
t_{u_{n}}-{ }^{t-1} u_{n}=t_{\Delta u_{n}} \text {, }
$$

we can write equation (4.5) as 


$$
{ }^{t-1} Q_{j n}{ }^{t} u_{n}=-{ }^{t-1} \psi_{j}
$$

with

$$
t_{u_{n}}={ }^{t-1} u_{n}+t_{\Delta u_{n}}
$$

Thus, starting with initial values for $U_{n}$ we can obtain increments in the displacement parameters $\Delta u_{n}$, by evaluating $\psi_{j}$ and $Q_{j n}$ at each increment. The solution is attained when the $\Delta_{\mathrm{n}}$ and $\psi_{j}$ are smaller than some prescribed tolerance for each of them; ideally both should be zero. From equations $(4.2)$ to $(4.4)$ we now obtain

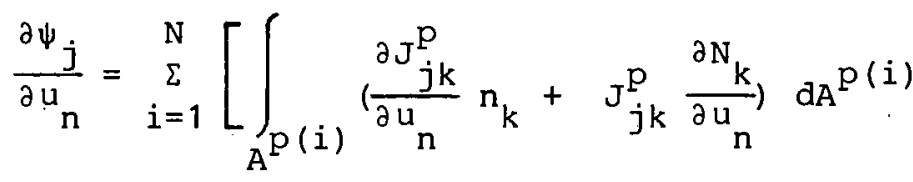

$$
\begin{aligned}
& \left.+\int_{A}\left(\frac{\partial J_{j k}^{S}}{\partial u_{n}} N_{k}+J_{j k}^{S} \frac{\partial N_{k}}{\partial u_{n}}\right) d A s(i)\right]
\end{aligned}
$$

where

$$
\left.\frac{\partial n_{k}}{\partial u_{n}}=\int_{-t_{p / 2}}^{t p / 2} D_{k \ell}^{p} \frac{\partial c_{\ell m}}{\partial \eta_{p}} \frac{\partial n_{p}}{\partial u_{n}} \eta_{m}+c_{\ell m} \frac{\partial \eta_{m}}{\partial u_{n}}\right) d z,
$$

$$
\frac{\partial N_{k}}{\partial u_{n}}=\int_{-t_{s}}^{t_{s} / 2} D_{k \ell}^{p} \quad\left(\frac{\partial C_{\ell m}}{\partial H_{p}} \frac{\partial H_{p}}{\partial U_{n}} H_{m}+C_{\ell m} \frac{\partial H_{p}}{\partial u_{n}}\right) d z ;
$$

here, $p$ ranges ${ }^{-t} / 2$ Irom 1 to 3 and the matrices are as defined in Chapter 3.

This full Newton-Raphson iteration is generally not very effective (Bathe and Cimento(1980)) since it is computationally expensive, requiring $\underline{\Psi}$ and $\underline{Q}$ to be evaluated at each iteration, and for solution effectiveness requires the use of relatively large load increments. It is more effective to use a 
modification of this algorithm. The modified Newton-Raphson procedure used in this analysis utilizes the matrix $\underline{Q}$ which is computed at the most recent equilibrium configuration and operates on the equations

$$
Q_{j n}{ }^{t} u_{n}=-{ }^{t-1} \psi_{j}
$$

where now only $\Psi$ is evaluated at each iteration. This procedure is shown for the one dimensional case in Fig. 4.1.

\subsubsection{Aitken Acceleration}

When there is a sudden softening in the system during a load increment the modified Newton-Raphson method requires a large number of iterations. This can be improved by using some acceleration scheme. The acceleration scheme used in this analysis is Aitken acceleration (Bathe and Cimento (1980)). With Aitken acceleration equation (4.7) becomes

$$
t_{u_{n}}={ }^{t-1} u_{n}+{ }^{t-1} \alpha_{n m} t_{\Delta d}
$$

where $\underline{\alpha}$ is a square diagonal matrix containing the acceleration factors for each of the displacement parameters ( $m$ ranges from 1 to 4 ). The individual acceleration factors are given by

$$
t-1 \alpha_{i i}=\frac{t-1 u_{i}}{t-1 u_{u_{i}}-{ }^{t} \Delta u_{i}} ; \alpha_{i j}=0 \text { if } i \neq j
$$

The derivation of and reason for these factors are shown in Fig 4.2. It is noted by Bathe and Cimento (1980) that this acceleration can only be applied every second iteration to avoid inaccurate estimates of these factors.

Difficulties arise with Aitken acceleration when the denominator of equation (4.13) for a particular displacement parameter is small. It is therefore more reliable to use a modification proposed by Jennings (1971) where a common acceleration factor is used for all the displacement parameters. Thus, instead of equations $(4.12)$ and $(4.13)$ we use

$$
t_{u_{n}}={ }^{t-1} u_{n}+t-1 s t_{\Delta u_{n}}
$$




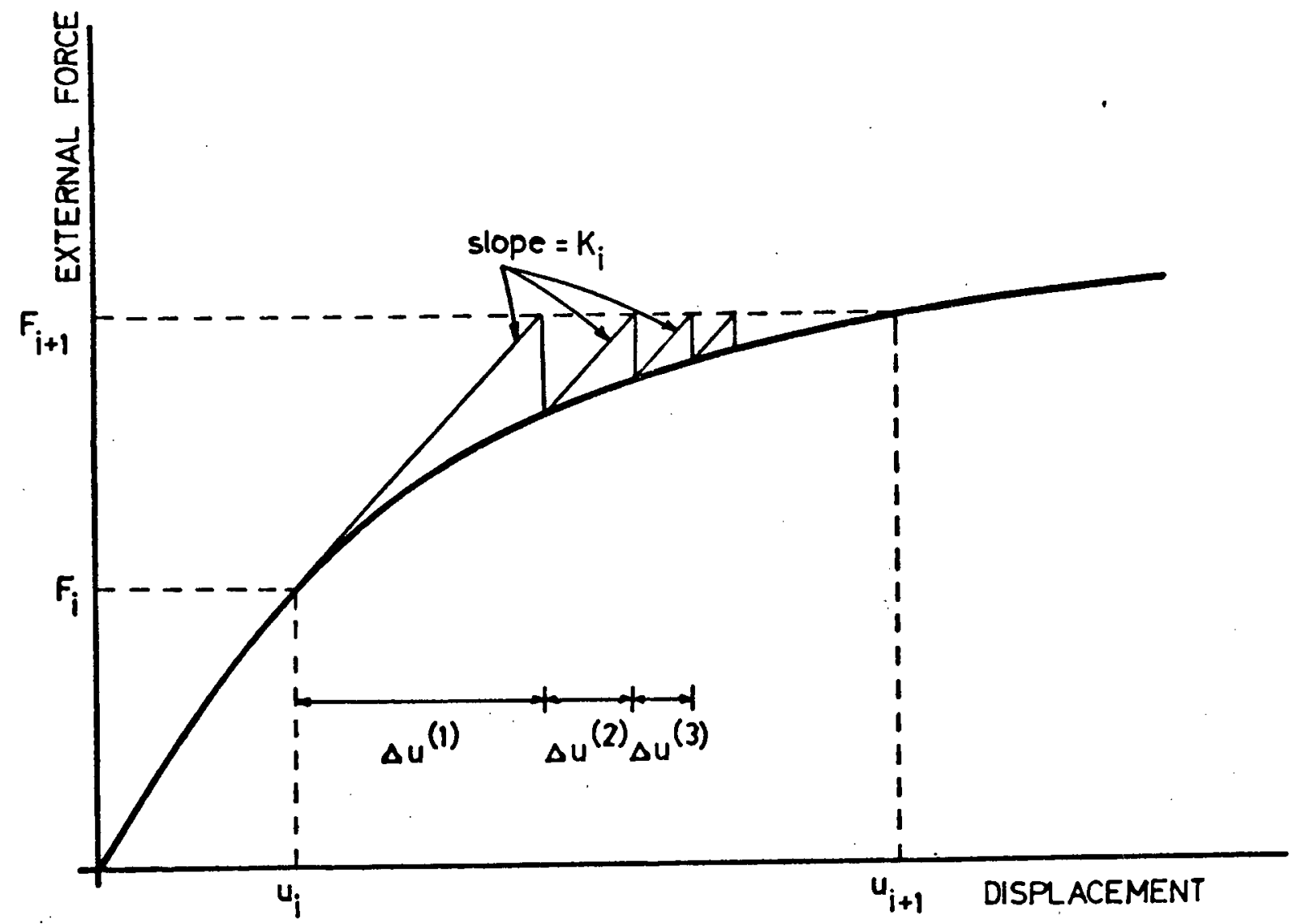

Fig. 4.1 Modified Newton-Raphson Iteration. 


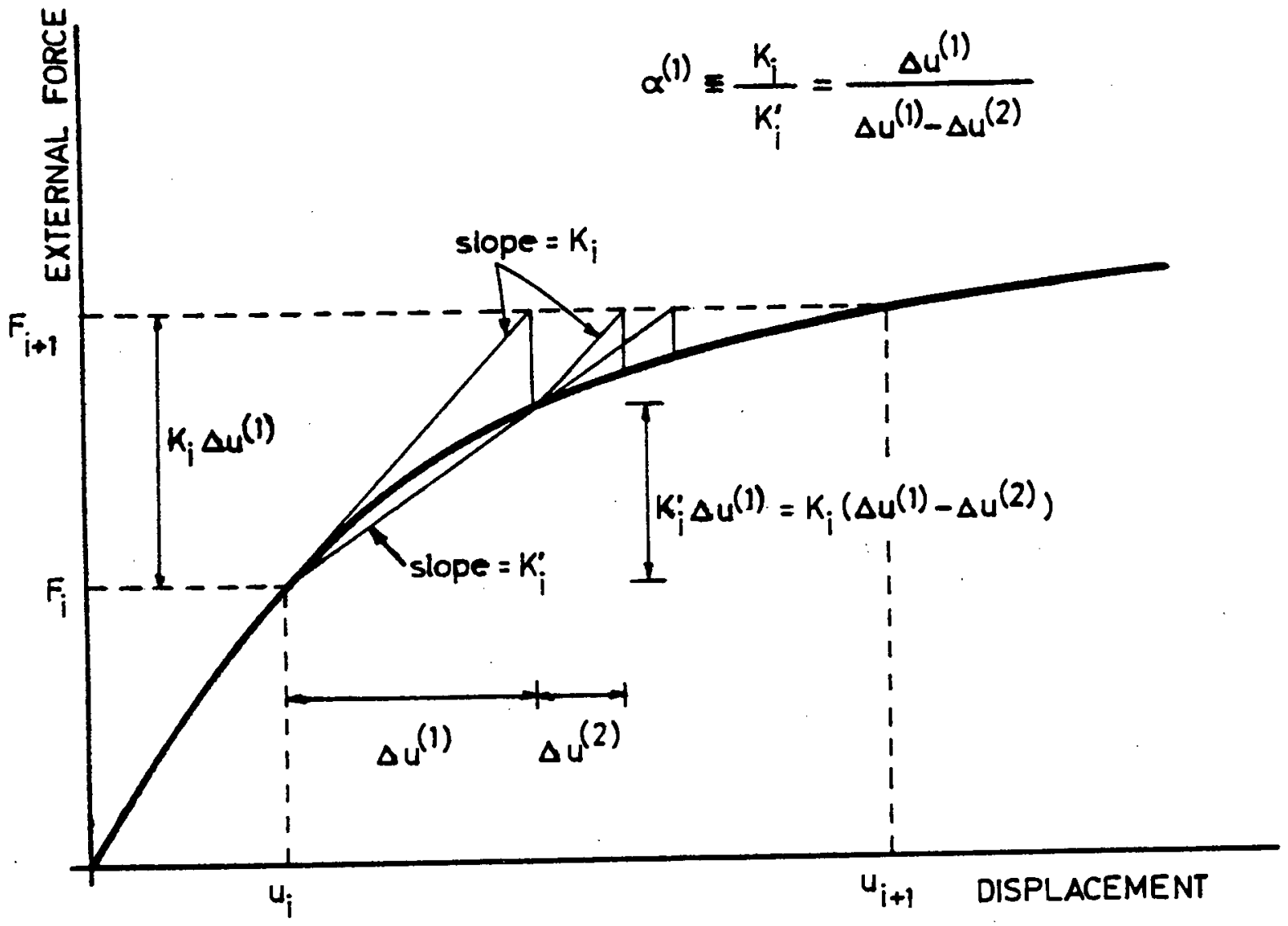

Fig. 4.2 Aitken Acceleration of the Modified Newton-Raphson Iteration. 


$$
t-1 u=\frac{{ }_{u} u_{n}{ }^{t-1} u_{n}}{t-1 u_{n}\left({ }^{t-1} \Delta u_{n}-t u_{n}\right)} ; \text { (sum on } n \text { ) }
$$

equations (4.14) and (4.15) are used every other iteration to accelerate the modified Newton-Raphson iteration.

For the present problem it was found that the system normally stiffened so that small increments were needed to obtain convergence (see also Bathe and Cimento(1980)). By neglecting the first term in equations (4.9) and (4.10), and thus by using a steeper slope than the tangent modulus, a more reliable and stable convergence to the solution was obtained.

\subsubsection{Automatic increment sizes}

It is desirable to have smaller increments of the loading parameter in the more non-linear regions of the analysis and larger increments in the more linear regions. The proposed formula to adjust the increment sizes according to the number of iterations previously used was given by Crisfield (1980). For this analysis the $\mathrm{q}^{\text {th }}$ load parameter increment is given by

$$
q_{\Delta 0_{1}}=q_{L u_{1}} \frac{I_{d}}{q-1 I}
$$

where ${ }^{q-1} \Delta u_{o_{1}}$ is the $(q-1)$ th load parameter increment; ${ }^{q-1} I$ is the number of iterations required at the $(q-1)$ th step and $I_{d}$ is the desired number of iterations. This equation inevitably leads to smaller increments in the more non-linear regions where more than the desired number of iterations have been encountered.

To ensure that very large increments are not obtained a maximum increment size is specified for the elastic range and a smaller maximum increment is specified for the plastic range.

\subsubsection{Solution of equations to obtain increments}

For each iteration the increments of the displacement parameters are obtained by solving equations (4.11). The matrix $\mathscr{Q}$ has been evaluated at the beginning of the load step and the vector $\psi$ is evaluated for each iteration. Thus, using equation (4.11), we obtain the increments ${ }^{t} u_{n^{*}}$ The procedure used in this analysis is Gauss elimination with full pivoting (Dahlquist and Björck (1974)). Full pivoting means that at each stage of the elimination procedure the largest value in the sub-matrix is placed in the first position 
on the diagonal, thus ensuring that errors are not introduced by small diagonal values. It should be noted that since the matrix $\underline{Q}$ is constant for each load increment, the upper triangular form has only to be computed once and the correct row operations performed on the $\psi$ vector at each iteration.

\subsection{Numerical Integration}

\subsubsection{Gaussian Quadrature}

The Gaussian integration formula for the one dimensional case can be written as

$$
\int_{-p}^{p} f(x) d x=p \sum_{j=1}^{n} H_{j} f\left(p a_{j}\right)
$$

where the weight coefficients $\mathrm{H}_{j}$ and the spacing coefficients $a_{j}$ are chosen according to the degree of polynomial that is being integrated. This equation, which requires $\mathrm{n}$ coefficients and sampling points; integrates exactly a polynomial of degree $(2 n-1)$. For an arbitrary interval this equation can be written as

$$
\begin{aligned}
\int_{c}^{d} f(x) d x & =\frac{d-c}{2} \int_{-1}^{1} f\left(\frac{d-c}{2} t+\frac{d+c}{2}\right) d t \\
& =\frac{d-c}{2} \sum_{j=1}^{n} H_{j} f\left(\frac{d-c}{2} a_{j}+\frac{d+c}{2}\right) .
\end{aligned}
$$

The most obvious way to evaluate a double integral is to evaluate the inner integral first, keeping the variable of the outer integral constant. Using this approach the two dimensional form of the Gaussian integration formula is as follows:

$$
\int_{-q}^{q} \int_{-p}^{p} f(x, y) d x d y=q p \sum_{i=1}^{m} \sum_{j=1}^{n} H_{i} H_{j} f\left(p a_{j}, q a_{i}\right) .
$$

This equation integrates a polynomial which is of order $(2 n-1)$ in the $x$ direction and of order $(2 m-1)$ in the $y$ direction exactly. Changing the 
interval as in equation (4.18) can also be done in both directions.

The formulae presented here are used for integration over the panel and stiffener midsurfaces and the coefficients are modified in order to integrate through the panel and stiffener thicknesses. The weight and spacing coefficients for these equations are given in Stroud and Secrest (1966).

\subsubsection{Integration over the midsurface areas}

It was found that, for an arbitrary number of axial half-waves and for one circumferential half-wave between two stiffeners, the contribution to the virtual work from each pair of adjacent panels and from each pair of circumferentially consecutive stiffeners was equal. If there are an even number of half-waves in the longitudinal direction then each panel and each stiffener have equal contributions as in Syngellakis and Walker (1978b). The internal virtual work integrals in equation (3.33), shown here without the integrands, can be written as

$$
\begin{aligned}
& \sum_{i=1}^{N}\left[\int_{A}(\ldots)^{p} d^{p(i)}+\int_{A}(\ldots(i))^{s} d A^{s(i)}\right] \\
& \left.=\sum_{i=1}^{2}\left[\int_{A}(\ldots)^{p} p_{d A} p(i)+\int_{A} s(i)\right)^{s} d A s(i)\right] .
\end{aligned}
$$

These two panels are subdivided into "elements" which span one half-wave in each direction and the stiffeners are divided into "elements" which span half-waves in the longitudinal direction. This is shown in Fig 4.3. It was found that alternate "elements" in the longitudinal direction had equal contributions and thus if we consider an even number of half-waves in this direction the right hand side of equation (4.20) can be written, for arbitrary $\mathrm{m}$, as

$$
m \beta\left[\int_{-\theta_{0}}^{\theta_{0}} \int_{0}^{\ell / m}(\ldots)^{\operatorname{p}} r d x d \theta+\int_{-\theta_{0}}^{\Theta_{0}} \int_{\ell / m}^{2 \ell / m}(\ldots)^{\operatorname{p}} r d x d \theta\right.
$$




$$
\left.+\int_{-d}^{d} \int_{0}^{\ell / m}(\ldots)^{s} d x d y+\int_{-d}^{d} \int_{\ell / m}^{2 \ell / m}(\ldots)^{s} d x d y\right]
$$

These four terms are contributions from the "elements" numbered 1 to 4 in Fig. 4.3.

It was further found that symmetries exist within these "elements". For the panels there are symmetries in both directions i.e.: about $\mathrm{x}=\ell / 2 \mathrm{~m}, 3 \ell / 2 \mathrm{~m}$ and $\theta=0$; for the stiffener "elements" there is only symmetry about $x=\ell / 2 \mathrm{~m}$, $3 \ell / 2 \mathrm{~m}$. Equation (4.21) thus becomes

$$
\begin{gathered}
m \beta\left[4 \int_{-\theta_{0}}^{0} \int_{0}^{\ell / 2 m}(\ldots)^{p} r d x d \theta+4 \int_{-\theta}^{0} \int_{\ell / m}^{3 \ell / 2 m}(\ldots)_{p} r d x d \theta\right. \\
\left.+2 \int_{-d}^{d} \int_{0}^{\ell / 2 m}(\ldots)_{s} d x d y+2 \int_{-d}^{d} \int_{\ell / m}^{3 \ell / 2 m}(\ldots)^{s} d x d y\right],
\end{gathered}
$$

which is integrated using the Gaussian quadrature formulae in Section 4.3.1. It was found that the use of 10 Gauss points in both directions over each "element" was sufficient for all cases considered.

\subsubsection{Integration through the thicknesses}

Points on the surfaces of the panels or stiffeners are likely to undergo plastic deformation first. This necessitates the inclusion of these surface points in the formula for numerical integration through the thickness. Simpson's rule is the simplest integration formula to use but this would need more sampling points than using Gauss's integration formula. However, the latter does not have sampling points on the surface. It would thus be an advantage to marry the two integration rules. This was achieved in the following manner: suppose $n$ sampling points are to be used to integrate a function. If the sampling and weight coefficients are arbitrary this produces $2 n$ unknowns. We then expand equation (4.17) in terms of these unknowns for the functions $f(x)=x^{a}$ where $a=0, \ldots, 2 n-1$ thus obtaining 


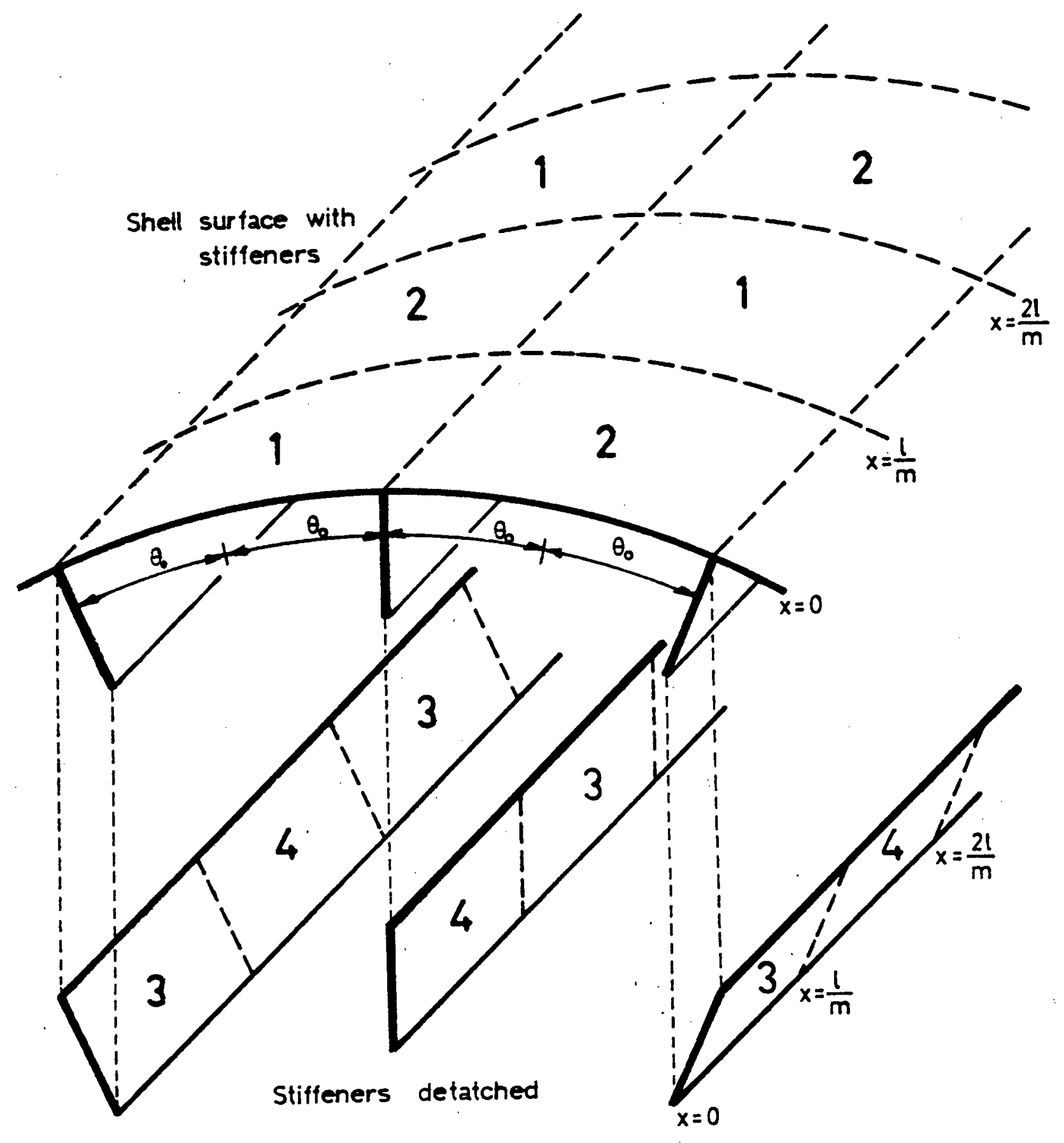

All "elements" with same number have equal contributions to internal virtual work.

Fig.4.3 Integration "elements". 
2n simultaneous equations in which the left hand side can be found exactly. Conditions of symmetry are then imposed on the sampling coefficients which reduces the number of equations. To obtain the coefficients for Gaussian quadrature these equations are then solved for the unknowns, but we impose a further condition in that the end points are to be fixed at the extremities of the interval, i.e.: sampling coefficients of +1 and -1 , and then these equations are solved to obtain the modified Gaussian coefficients.

Fixing two points in this way reduces by two the order of the polynomial than is exactly integrated witn $n$ sampling points. Thus we can obtain integrations rules which integrate a polynomial of degree $(2 n-3)$ by using $n$ sampling points. Table 4.1 shows integration rules obtained in this way for different orders of polynomial integration. It should be noted that the rule for a $3^{\text {rd }}$ order polynomial reduces to simpson's rule. These modified integration rules use the same formulae as in section 4.3 .1 , but with coefficients from the Table 4.1; they are employed in this analysis for the integration through the thicknesse:s of the panels and stiffeners.

TABLE 4.1 Weight and Sampling coefficients for n-point modified Gaussian quadrature formula to integrate polynomials of order $(2 n-3)$.

$$
\int_{-1}^{1} f(x) d x=\sum_{j=1}^{n} H_{j} f\left(a_{j}\right)
$$

\begin{tabular}{|ccc|}
\hline $\pm a$ & & $H$ \\
\hline & $n=3$ (Simpson's rule) & \\
1 & & $1 / 3$ \\
0 & & $4 / 3$ \\
& $n=4$ & \\
$\sqrt{1 / 5}$ & & $1 / 6$ \\
1 & $n=5$ & $5 / 6$ \\
$\sqrt{3 / 7}$ & & $1 / 10$ \\
0 & & $49 / 90$ \\
\hline
\end{tabular}




\subsection{Iteration for Secant Modulus}

Once any point in a panel or stiffener has yielded it is necessary to obtain the secant modulus $E_{S}$, at this position. The equation for the secant modulus $\mathrm{E}_{\mathrm{S}^{\prime}}$ as shown in Section 3.4 is given by

$$
\frac{\bar{n}}{\bar{\sigma}}=\frac{1}{E_{s}}=\frac{\sigma}{\bar{\sigma} E}\left[\frac{1}{n}\left(\frac{\bar{\sigma}_{\sigma}}{\sigma_{y}} n+\frac{n-1}{n}\right]\right.
$$

Thus, to obtain $E_{S}$ at a point we require the value of $\vec{\sigma}$ which is in turn dependant on $\mathrm{E}_{\mathrm{s}}$ and $\nu_{\mathrm{s}}$. Therefore, by knowing the strains, it is possible to iterate in order to obtain $\bar{\sigma}$ and $E_{s}$. Using the first part of equation (4.24), the equation for total effective stress (3.16) and the constitutive equation (3.25), we are able to write the total effective strain in terms of the strains at that point as

$$
\begin{array}{r}
\vec{n}=\frac{1}{\left(1-v_{s}^{2}\right)}\left[\left(1+v_{s}^{2}\right)\left(n_{1}^{2}+n_{2}^{2}-\eta_{1} \eta_{2}+3 \eta_{12}^{2}\right)\right. \\
\left.-v_{s}\left(\eta_{1}^{2}+\eta^{2}-4 \eta_{1} \eta_{2}+6 \eta_{12}^{2}\right)\right]^{\frac{3}{2}} ;
\end{array}
$$

also, inverting equation $(4.24)$ we obtain

$$
\vec{\sigma}=\sigma_{y}\left[1-n+n \frac{E}{\sigma_{Y}} \bar{n}\right]^{1 / n}
$$

In this analysis the following iteration procedure is followed:

(i) $\eta_{1}, \eta_{2}$ and $\eta_{12}$ are evaluated at any stage of loading and any position; a first approximation of $\mathrm{E}_{\mathrm{S}}$ and thus $\nu_{S}$ is also chosen (either the elastic value or the last known value);

(ii) using $v_{s}$, obtain $\bar{n}$ from equation (4.25);

(iii) use equation (4.26) to obtain $\bar{\sigma}$;

(iv) compute $\mathrm{E}_{\mathrm{S}}=\vec{\sigma} / \bar{\eta}$ and $\nu_{\mathrm{S}}$;

(v) repeat steps (ii) to (iv) until the value for $\mathrm{E}_{\mathrm{s}}$ converges.

Note that this iterative procedure is only followed if yielding has occurred at the particular point in the panel or stiffener.

$$
4.5 / \quad \cdots+
$$




\subsection{Concluding Remarks}

By combining the procedures described in this Chapter we can numerically obtain all solutions on the primary path. The complete numerical implementation used in this analysis is given in the program flow chart in Fig. 4.4 .

Results obtained using these procedures are discussed in Chapter 7. 


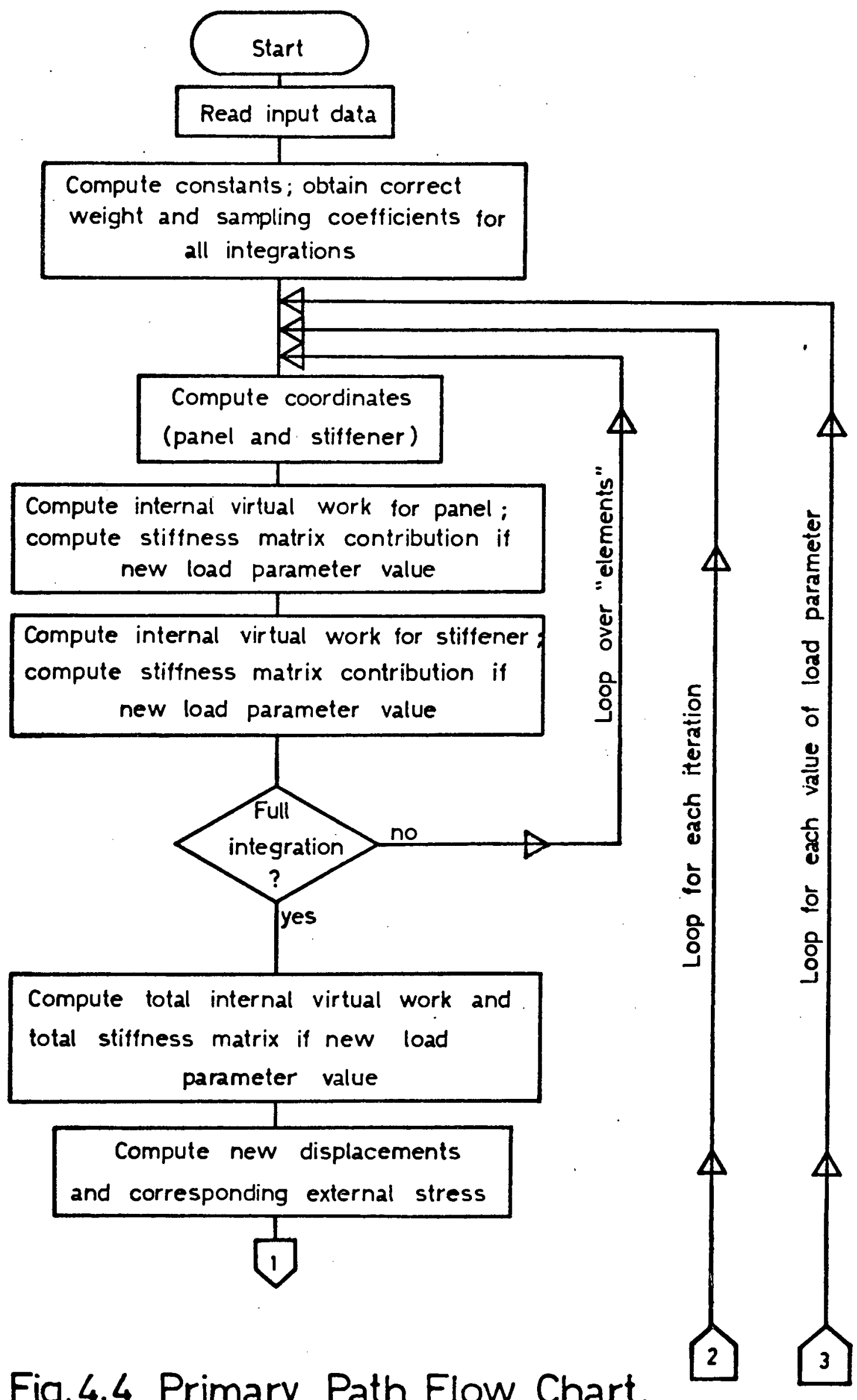

Fig.4.4 Primary Path Flow Chart. 


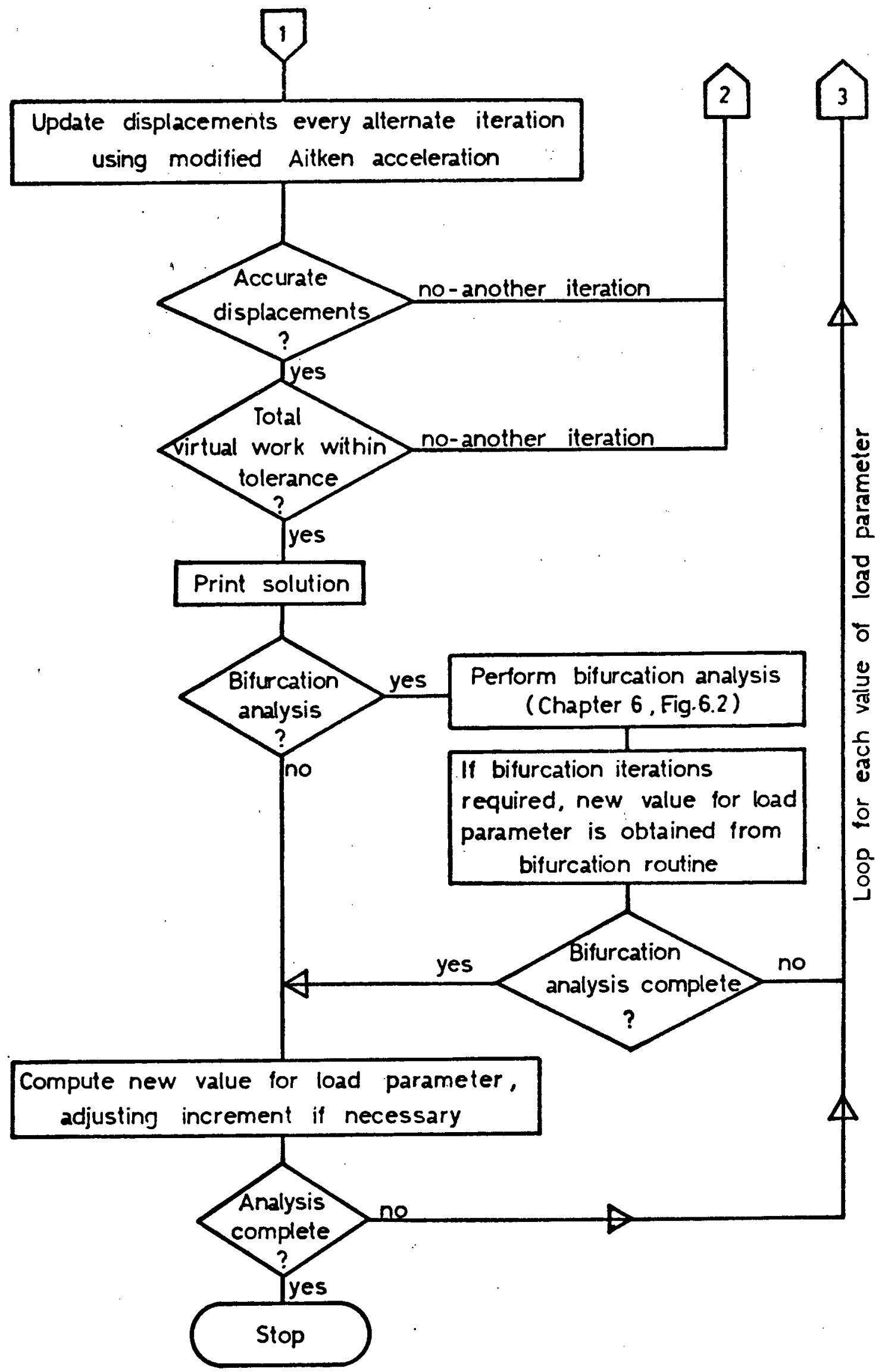

Fig.4.4 Primary Path Flow Chart (continued). 


\section{CHAPTER 5}

\section{BIFURCATION ANALYSIS}

\subsection{Introductory Remarks}

The primary equilibrium path has already been discussed in Chapters 3 and 4. In this chapter we investigate the possibility of bifurcation (a branching of the equilibrium path) and set out the theory and equations for determining loads at which bifurcation occurs. The incremental relations described in section 5.2, in which we also derive the instantaneous moduli for a rate constitutive equation using $J_{2}$ deformation theory; are used to develop the criterion for bifurcation in Section 5.3 as given by Hutchinson (1974).

In Section 5.4 a bifurcation mode is assumed and the relevant substitutions carried out in order to obtain a criterion for bifurcation in terms of a finite number of eigenmodal displacement parameters.

\subsection{Incremental Relations}

\subsubsection{Incremental strain-displacement relations}

At any stage of loading the deformation is characterised by the deform-

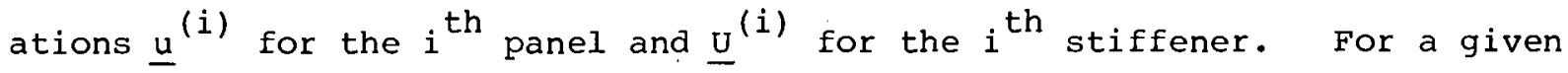
increment in load at some stage in the loading process the solution for the displacement increments are given by $\underline{\dot{u}}^{(i)}$ and $\underline{\dot{U}}^{(i)}$ for the $i{ }^{\text {th }}$ panel and stiffener, respectively. It is possible to obtain the incremental midsurface membrane and bending strains for the panels and stiffeners from the strain-displacement relations (3.7) to (3.10). Thus, for the typical panel

$$
\begin{aligned}
\dot{\epsilon}_{\alpha \beta}\left(x_{1}, x_{2}\right) & =\frac{1}{2}\left(\dot{u}_{\alpha, \beta}+\dot{u}_{\beta, \alpha}\right)-b_{\alpha \beta} \dot{w} \\
& +\frac{1}{2}\left(w_{, \alpha} \dot{w}_{, \beta}+\dot{w}_{, \alpha}{ }_{, \beta}+\bar{w}_{, \alpha} \dot{w}_{, \beta}+w_{, \alpha} \bar{w}_{, \beta}\right), \\
\dot{\mathcal{u}}_{\alpha \beta}\left(x_{1}, x_{2}\right) & =\dot{w}_{, \alpha \beta} ;
\end{aligned}
$$


for a typical stiffener

$$
\begin{aligned}
& \dot{\mathrm{E}}_{\alpha \beta}\left(\mathrm{X}_{1}, \mathrm{X}_{2}\right)=\frac{1}{2}\left(\dot{\mathrm{U}}_{\alpha, \beta}+\dot{\mathrm{U}}_{\beta, \alpha}\right) \\
&+\frac{1}{2}\left(\mathrm{~W}_{, \alpha} \dot{\mathrm{W}}_{, \beta}+\dot{\mathrm{W}}_{, \alpha} \mathrm{W}_{, \beta}+\overline{\mathrm{W}}_{, \alpha} \dot{\mathrm{W}}_{, \beta}+\dot{\mathrm{W}}_{, \alpha} \overline{\mathrm{W}}_{, \beta}\right), \\
& \dot{\mathrm{K}}_{\alpha \beta}\left(\mathrm{x}_{1}, \mathrm{x}_{2}\right)=\dot{\mathrm{W}}_{, \alpha \beta} .
\end{aligned}
$$

The incremental strains at any point through the thicknesses for a typical panel and stiffener are:

$$
\begin{aligned}
& \dot{\eta}_{\alpha \beta}\left(x_{1}, x_{2}, z\right)=\dot{\varepsilon}_{\alpha \beta}-z \dot{x}_{\alpha \beta}, \\
& \dot{H}_{\alpha \beta}\left(x_{1}, x_{2}, z\right)=\dot{E}_{\alpha \beta}-z \dot{K}_{\alpha \beta} .
\end{aligned}
$$

\subsubsection{Incremental Constitutive Relations}

For a given strain increment (or rate) the three dimensional rate constitutive relation is

$$
\dot{\sigma}_{i j}=\bar{L}_{i j k \ell} \dot{\eta}_{k \ell}
$$

where, in the present problem, the instantaneous moduli $\bar{L}_{i j k \ell}$ correspond to those for small-strain deformation theory of plasticity. The moduli are thus dependant upon the total strains (or total stresses) at the current state of loading. For a state of approximate plane stress this equation may be written in the form

$$
\dot{\sigma}_{\alpha \beta}=L_{\alpha \beta \kappa \gamma} \dot{\eta}_{K \gamma}
$$

where $\alpha$ and $\beta$ range from 1 to 2 . The coefficients $L_{\alpha \beta u \gamma}$ are obtained from

$$
\mathrm{L}_{\alpha \beta u \gamma^{\prime}} \quad \cdots \cdots
$$




$$
L_{\alpha \beta K \gamma}=\bar{L}_{\alpha \beta K \gamma}-\frac{\bar{L}_{\alpha \beta \text { 3 3 }} \bar{L}_{33 K \gamma}}{\bar{L}_{3333}} .
$$

This relation is obtained in the same way as the coefficients $C_{\alpha \beta \mu \gamma}$ in Section 3.4 .

The proceedure for obtaining $\mathrm{L}_{\alpha \beta \mathcal{H}}$ is as follows: for a state of approximate plane stress the total strain-total stress equations are

$$
n_{\alpha \beta}=\frac{1+\nu_{S}}{E_{S}} \sigma_{\alpha \beta}-\frac{\nu_{S}}{E_{S}} \sigma_{\gamma \gamma} \delta_{\alpha \beta}
$$

where subscripts range from 1 to 2 and

$$
\begin{aligned}
\delta_{\alpha \beta} & =1 \quad \alpha=\beta \\
& =0 \quad \alpha \neq \beta .
\end{aligned}
$$

The incremental form of this equation is

$$
\begin{aligned}
\dot{\eta}_{\alpha \beta}= & \frac{1+\nu_{s}}{E_{s}} \dot{\sigma}_{\alpha \beta}+\frac{\dot{\nu}_{s}}{E_{s}} \sigma_{\alpha \beta}+\left(\frac{1}{E_{s}}\right)\left(1+\nu_{s}\right) \sigma_{\alpha \beta} \\
& -\left[\frac{\nu_{s}}{E_{S}} \dot{\sigma}_{Y Y}-\frac{\dot{\nu}_{s}}{E_{S}} \sigma_{Y Y}-\left(\frac{1}{E_{S}}\right) \dot{\nu}_{S} \sigma_{\gamma Y}\right] .
\end{aligned}
$$

Noting that (from Section 3.4)

$$
\begin{aligned}
& E_{s}=\bar{\sigma} / \bar{\eta}, \\
& \nu_{s}=\frac{1}{2}+\frac{E_{S}}{E}\left(\nu-\frac{1}{2}\right), \\
& \bar{\sigma}^{2}=\frac{3}{2} \sigma_{i j}{ }^{\prime} \sigma_{i j},
\end{aligned}
$$

and that the tangent modulus $\mathrm{E}_{\mathrm{T}}$ is defined as

$$
\mathrm{E}_{\mathrm{T}}=\dot{\bar{\sigma}} / \frac{\dot{n}}{\mathrm{n}}
$$


the following incremental quantities can be obtained

$$
\begin{aligned}
& \left.\left(\frac{1}{\mathrm{E}_{\mathrm{S}}}\right)=\left(\frac{1}{\mathrm{E}_{\mathrm{T}}}\right)-\frac{1}{\mathrm{E}_{\mathrm{S}}}\right) \frac{\dot{\bar{\sigma}}}{\bar{\sigma}}, \\
& \dot{\mathrm{E}}_{\mathrm{S}}=\left(\mathrm{E}_{\mathrm{T}}-\mathrm{E}_{\mathrm{S}}\right) \frac{\mathrm{E}_{\mathrm{S}}}{\mathrm{E}_{\mathrm{T}}} \frac{\dot{\bar{\sigma}}}{\bar{\sigma}}, \\
& \dot{v}_{\mathrm{S}}=-\left(\frac{1}{\mathrm{E}_{\mathrm{T}}}-\frac{1}{\mathrm{E}_{\mathrm{T}}}\right) \frac{\mathrm{E}_{\mathrm{S}}^{2}}{\mathrm{E}^{2}} \frac{\dot{\bar{\sigma}}}{\frac{\sigma}{\sigma}}\left(v-\frac{1}{2}\right), \\
& \dot{\bar{\sigma}}=\frac{3}{2 \bar{\sigma}} \dot{\sigma}_{i j}{ }^{\prime} \sigma_{i j},
\end{aligned}
$$

where $i$ and $j$ range from 1 to 3 .

By combining equations (5.12) to (5.15) with equation (5.11) the following incremental strain-stress relation is obtained:

$$
\dot{\eta}_{\mu \gamma}=L_{\alpha \beta \mu \gamma}^{-1} \dot{\sigma}_{\alpha \beta}
$$

where $L_{\alpha \beta \mathcal{}}^{-1}$ is symmetric. Since $\dot{\eta}_{12}=\dot{\eta}_{21}$ and $\dot{\sigma}_{12}=\dot{\sigma}_{21}$ this equation can be written in the following forms; for a typical panel

$$
\underline{\dot{n}}=\underline{\mathrm{p}}^{-1} \dot{\sigma}^{\mathrm{p}}
$$

and for a typical stiffener

$$
\underline{\dot{H}}=\underline{s}^{-1} \underline{\dot{\sigma}}^{s} \text {. }
$$

These equations are of the same form as those used for example by Gellin (1979). Here $\underline{\mathrm{P}}^{-1}$ and $\underline{\mathrm{s}}^{-1}$ maintain symmetry while

$$
\begin{aligned}
& \dot{n}=\left(\dot{n}_{x x^{\prime}} \dot{n}_{\theta \theta}, 2 \dot{n}_{x \theta}\right)^{T}, \\
& \dot{\sigma}^{p}=\left(\dot{\sigma}_{x x^{\prime}}, \dot{\sigma}_{\theta \theta^{\prime}}, \dot{\sigma}_{x \theta}\right)^{T},
\end{aligned}
$$


$\left.\stackrel{\dot{\mathrm{H}}}{=}=\dot{\mathrm{H}}_{\mathbf{x x}}, \dot{\mathrm{H}}_{\mathrm{YY}}, \quad \dot{\mathrm{H}}_{\mathrm{XY}}\right)^{\mathrm{T}}$,

$\dot{\sigma}^{s}=\left(\dot{\sigma}_{x x^{\prime}}, \dot{\sigma}_{Y y^{\prime}}, \dot{\sigma}_{x y}\right)^{T}$

and 

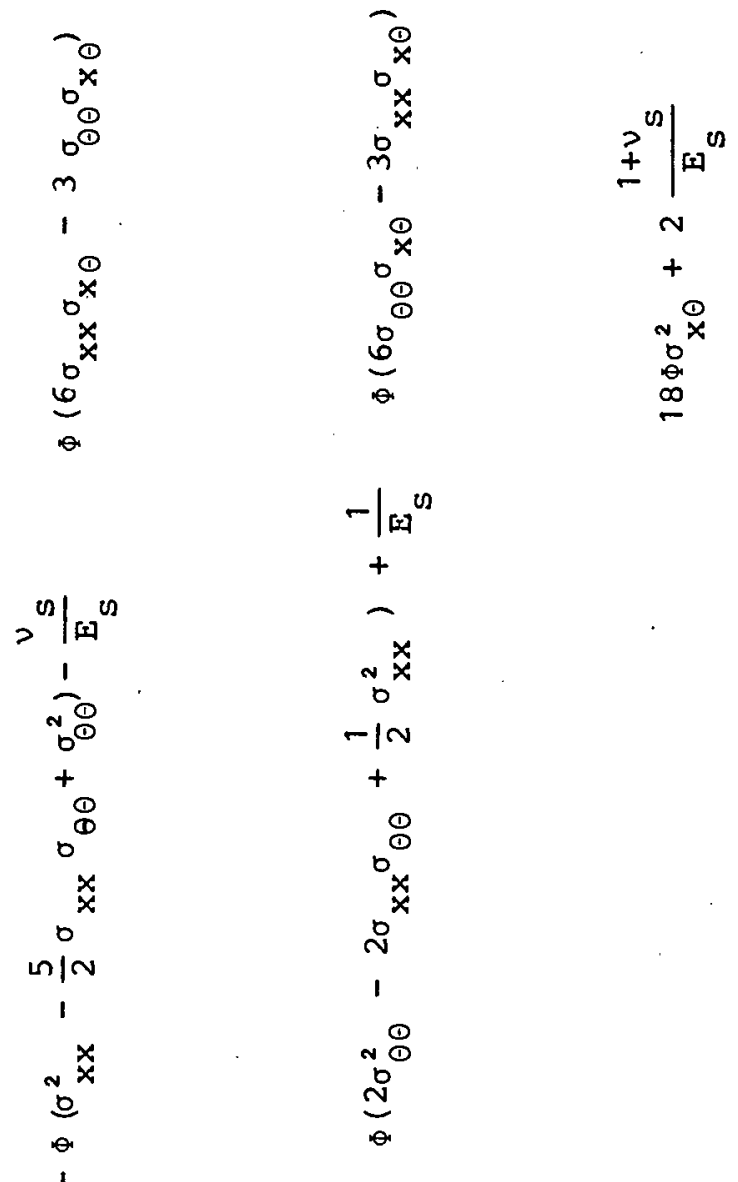

$-\left.\right|^{n}{ }^{2}$

$x_{x}^{x}$

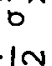

$x_{x}^{\circ}$

i

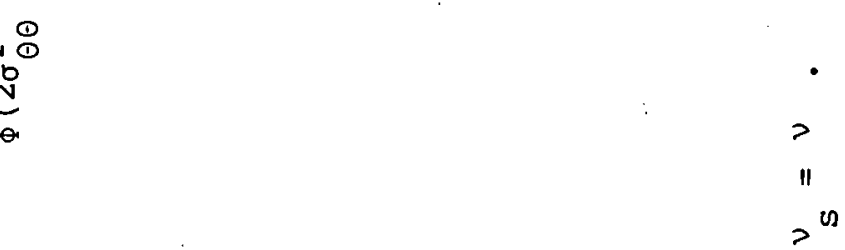

$-\operatorname{los}^{0}$

$+$

กิ

$-\mid \stackrel{N}{i}$

$-1 n$

+

崩

$-1 a^{2}$

$-10$

11

$\bullet$

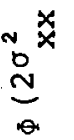

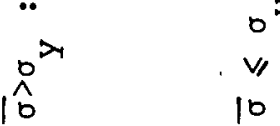

嵌 

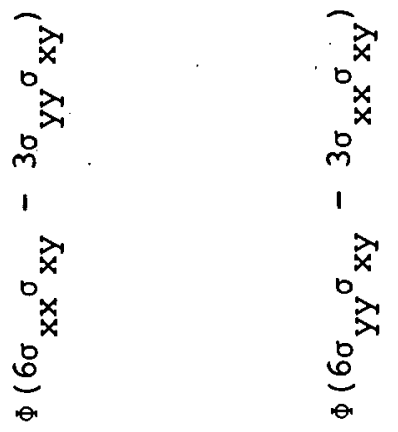

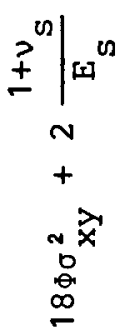
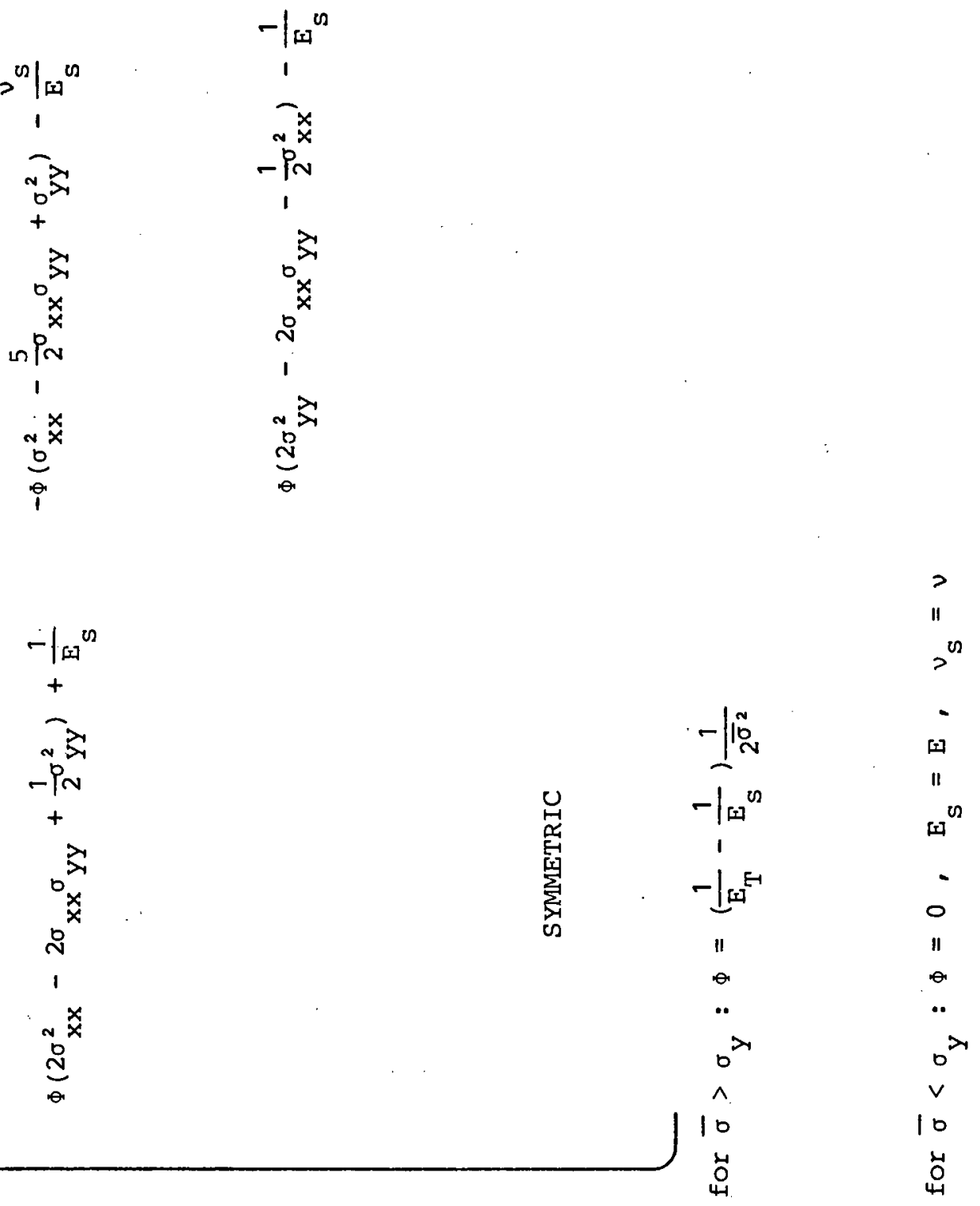

in 


\subsubsection{Incremental Stress Resultants}

Incremental stress resultants can be obtained from equations (3.27) and (3.28). Thus the membrane stress and bending moment resultants are defined as follows; for a typical panel,

$$
\dot{n}_{\alpha \beta}=\int_{-t_{p / 2}}^{t_{p} / 2} \dot{\sigma}_{\alpha \beta}^{p} d z \quad, \dot{m}_{\alpha \beta}=-\int_{-t}^{t} z / 2
$$

and for a typical stiffener,

$$
\dot{\mathrm{N}}_{\alpha \beta}=\int_{-t_{s / 2}}^{\mathrm{t}} \dot{\sigma}_{\alpha \beta}^{s / 2} \mathrm{dz} \quad, \dot{\mathrm{M}}_{\alpha \beta}=-\int_{-\mathrm{t}_{s / 2}}^{\mathrm{t}} \mathrm{z} \dot{\sigma}_{\alpha \beta}^{\mathrm{s}} \mathrm{dz} .
$$

The column vectors of membrane stress and bending moment resultants may be written in the following form for a typical panel and typical stiffener respectively

$$
\begin{aligned}
& \dot{\underline{n}}=\int_{-t}^{t} \int^{t} \underline{D}^{p} \dot{\sigma}^{p} d z, \\
& \dot{N}=\int_{-t}^{t} \underline{D}_{s / 2}^{t} \underline{\dot{\sigma}}^{s} d z,
\end{aligned}
$$

where $\underline{D}^{p}$ and $\underline{D}^{S}$ are given by equations (3.29) and (3.30), and

$$
\begin{aligned}
& \underline{\underline{n}}=\left(\dot{\mathrm{n}}_{x x^{\prime}}, \dot{\mathrm{n}}_{\theta \theta}, \dot{\mathrm{n}}_{x \theta^{\prime}}, \dot{\mathrm{m}}_{x x^{\prime}}, \dot{\mathrm{m}}_{\theta \theta}, \dot{\mathrm{m}}_{x \theta}\right)^{\mathrm{T}}, \\
& \dot{\mathrm{N}}=\left(\dot{\mathrm{N}}_{\mathrm{Xx}}, \dot{\mathrm{N}}_{\mathrm{Y} y^{\prime}}, \dot{\mathrm{N}}_{\mathrm{XY}}, \dot{\mathrm{M}}_{\mathrm{Xx}}, \dot{\mathrm{M}}_{Y Y^{\prime}}, \dot{\mathrm{M}}_{\mathrm{XY}}\right)^{\mathrm{T}} \text {. }
\end{aligned}
$$




\subsection{Bifurcation Criterion}

At any stage of deformation characterised by $\underline{u}^{(i)}$ and $\underline{U}^{(i)}$ for the $i^{\text {th }}$ panel and stiffener respectively, suppose that bifurcation is possible so that for a given increment of load there are at least two solutions for the displacement increments : $\underline{\dot{u}}^{\mathrm{a}}$ and $\underline{\dot{u}}^{\mathrm{b}}$ for a typical panel with their associated $\underline{\dot{U}}^{\mathrm{a}}$ and $\dot{\cup}^{b}$ for a typical stiffener. Incremental midsurface membrane and bending strains corresponding to these two solutions can be obtained from equations (5.1) to (5.4). It is therefore possible to obtain the incremental strains at any point through the thickness via equations (5.5) and (5.6) and thus the incremental stress quantities for the two solutions can be obtained as in equation (5.8): $\dot{\sigma}_{\alpha \beta}^{*}{ }_{\alpha \beta}^{p a}, \dot{\sigma}_{\alpha \beta}{ }_{\alpha \beta}^{p b}$ for a typical panel and $\dot{\sigma}_{\alpha \beta}^{*}, \dot{\sigma}_{\alpha \beta}{ }_{\alpha \beta}$ fb for a typical stiffener.

We can now introduce the following differences between the two solutions. For a typical panel

$$
\underline{\tilde{u}}, \tilde{\varepsilon}_{\alpha \beta}, \tilde{\mathcal{H}}_{\alpha \beta} \text { and } \tilde{\sigma}_{\alpha \beta}^{\star p} \text {, }
$$

and for a typical stiffener.

$$
\underline{\underline{U}}, \widetilde{\mathrm{E}}_{\alpha \beta}, \widetilde{\mathrm{K}}_{\alpha \beta} \text { and } \tilde{\sigma}_{\alpha \beta}^{\star^{5}},
$$

where

$$
(\ldots)=(\ldots)^{a}-(\ldots)^{b}
$$

From these quantities we can obtain $\tilde{n}_{\alpha \beta}, \tilde{H}_{\alpha \beta}$ as in equations (5.5) and (5.6), and also the bending moment and membrane stress resultants $\tilde{n}_{\alpha \beta}$, $\tilde{m}_{\alpha \beta}$, $\tilde{\mathbb{N}}_{\alpha \beta}$ and $\tilde{\mathbb{M}}_{\alpha \beta}$ from $\tilde{\sigma}_{\alpha \beta}^{*}$ and $\tilde{\sigma}_{\alpha \beta}^{*}$ as in equations (5.19) and (5.20).

Within the context of Donnell-Mushtari-vlasov shell theory and the present problem if both solution rates are possible solutions it must follow that (Hutchinson(1974))

$$
H \equiv \sum_{i=1}^{N}\left\{\int_{A}(i) \tilde{n}_{\alpha \beta}^{*} \tilde{\varepsilon}_{\alpha \beta}+\tilde{m}_{\alpha \beta}^{*} \tilde{u}_{\alpha \beta}+n_{\alpha \beta} \tilde{w}_{, \alpha} \tilde{w}_{, \beta}\right) d A(i)
$$




$$
\left.+\int_{A}\left(\tilde{N}_{\alpha \beta}^{\star} \tilde{E}_{\alpha \beta}+\tilde{M}_{\alpha \beta} \tilde{\mathrm{K}}_{\alpha \beta}+\mathrm{N}_{\alpha \beta} \tilde{\mathrm{W}}_{, \alpha} \tilde{\mathrm{W}}_{, \beta}\right) d A \mathrm{~S}(i)\right\}=0,
$$

where $n_{\alpha \beta}$ and $\mathrm{N}_{\alpha \beta}$ are the current membrane stress resultants.

In the current state we obtain the moduli $L_{\alpha \beta \mu \gamma}^{c p}$ and $L_{\alpha \beta \mu \gamma}^{c s}$ corresponding to plastic loading for the panels and stiffeners, respectively, from equations $(5.8)$ to $(5.18)$. By using these so-called comparison moduli, comparison stress increment differences $\tilde{\sigma}_{\alpha \beta}^{\mathrm{p}}$ and $\tilde{\sigma}_{\alpha \beta}^{\mathrm{s}}$ can be found from which comparison bending moment and membrane stress resultant increment differences are obtained using equations (5.19) and (5.20): $\tilde{n}_{\alpha \beta}, \tilde{m}_{\alpha \beta}$ for a typical panel and $\tilde{\mathrm{N}}_{\alpha \beta}$, $\tilde{M}_{\alpha \beta}$ for a typical stiffener. These comparison moduli are given in equations (5.17) and (5.18) for the panels and stiffeners respectively. We now introduce the following quadratic functional as in Hutchinson (1974):

$$
\begin{aligned}
& F \equiv \stackrel{i}{\Sigma}_{=}^{N}\left\{\int_{A}\left(\tilde{n}_{\alpha \beta} \tilde{\varepsilon}_{\alpha \beta}+\tilde{m}_{\alpha \beta} \tilde{x}_{\alpha \beta}+n_{\alpha \beta} \tilde{w}_{, \alpha} \tilde{w}_{, \beta}\right) d A p(i)\right. \\
& \left.+\int_{A}\left(\tilde{N}_{\alpha \beta} \tilde{E}_{\alpha \beta}+\tilde{M}_{\alpha \beta} \tilde{\mathrm{K}}_{\alpha \beta}+\mathrm{N}_{\alpha \beta} \tilde{\mathrm{W}}_{, \alpha} \tilde{\mathrm{W}}_{, \beta}\right) d A(i)\right\} .
\end{aligned}
$$

It is shown (Hutchinson(1974)) that $\mathrm{H} \geqslant F$ and that the condition $F>0$

- for all admissable solutions ensures uniqueness of the solution increment. For bifurcation to be possible it is necessary that $\mathrm{H}$ vanishes when $F$ does. Hutchinson (1974) also shows that both solutions considered are possible when $\mathrm{F}=0$ if and only if both solution rates satisfy total loading (no elastic unloading) through the shell thickness. The variational statement $\delta F=0$ leads to an eigenvalue problem, the lowest eigenvalue corresponding to the bifurcation load. Thus we seek the external axial load for which the following equation is true: 


$$
\delta F=\sum_{i=1}^{N}\left\{\int_{A}(i) \tilde{n}_{\alpha \beta} \delta \tilde{\varepsilon}_{\alpha \beta}+\tilde{m}_{\alpha \beta} \delta \tilde{u}_{\alpha \beta}+n_{\alpha \beta} \tilde{w}_{, \alpha} \delta \tilde{w}_{, \beta}\right) d A^{p(i)}
$$

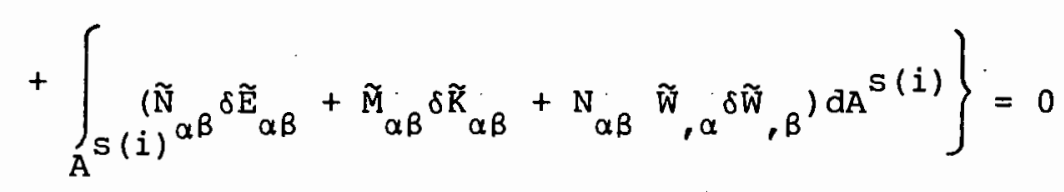

Substitution of the strain-displacement relations into the variational quantities of this equation and use of Green's theorem in the same way as in Section 3.6 would yield the governing equations and boundary conditions for this eigenvalue problem.

\subsection{Bifurcation Mode}

\subsubsection{Displacements}

The differences in displacement increment between the primary path and the bifurcated path are assumed for the $i^{\text {th }}$ panel to be

$$
\begin{aligned}
& \tilde{u}^{(i)}=u_{1}^{(i)} \cos \gamma \xi \sin \beta \rho \theta^{(i)}, \\
& \tilde{v}^{(i)}=v_{1}^{(i)} \sin \gamma \xi \cos \beta \rho \theta^{(i)}, \\
& \tilde{w}^{(i)}=w_{1}^{(i)} \sin \gamma \xi \sin \beta \rho \theta^{(i)}
\end{aligned}
$$

with

$$
\begin{aligned}
& u_{1}^{(i+1)}=u_{1}^{(i)}, \\
& v_{1}^{(i+1)}=v_{1}^{(i),} \\
& w_{1}^{(i+1)}=w_{1}^{(i),}
\end{aligned}
$$

Here $\gamma=q \pi r / \ell$ with $q$, the number of half-waves in the axial direction, being an arbitrary integer. The number of half-waves between two stiffeners in the 
circumferential direction $p$, is an even integer. This is explained in Appendix 5A. This mode is antisymetric with respect to the centre of the $i^{\text {th }}$ panel $\theta^{(i)}=0, i . e$.

$$
\begin{aligned}
& \tilde{u}^{(i)}(x, \theta)=-\tilde{u}^{(i)}(x,-\theta), \\
& \tilde{v}^{(i)}(x, \theta)=-\tilde{v}^{(i)}(x, \theta), \\
& w^{(i)}(x, \theta)=-w^{(i)}(x, \theta) .
\end{aligned}
$$

The corresponding solutions for the $i^{\text {th }}$ stiffener are obtained from the continuity conditions (3.6) which, being linear, are the same for the incremental displacement quantities. Thus

$$
\begin{aligned}
& \tilde{u}^{(i)}=0, \\
& \tilde{v}^{(i)}=0, \\
& w^{(i)}=(-1)^{p / 2}\left[v_{1}^{(i)}-\frac{1}{r}(y-e) \beta p w_{1}^{(i)}\right] \sin \gamma \xi .
\end{aligned}
$$

These relations are derived in Appendix 5A. The displacement differences in equations (5.26) and (5.29) for the panels and stiffeners respectively are, for the case when $p=2$, the same as those used by syngellakis and walker (1978b) .

\subsubsection{Strains at any point}

The differences in strain increment between the two equilibrium paths can be obtained by substituting the displacements from equations (3.38) to (3.45) and the displacement differences from equations $(5.26),(5,29)$ into the incremental strain-displacement relations (5.1) to (5.4) and then by using equations (5.5) and (5.6) for the panels and stiffeners respectively. The midsurface membrane and bending strain differences are shown in Appendix $5 \mathrm{~B}$ for a typical panel and stiffener. These relations are precisely those used by syngellakis and walker $(1978 \mathrm{~b})$ if $p=2$. The incremental strains as functions of position are given by the following equations : for a typical panel. 


$$
\underline{\tilde{n}}=\underline{M}^{\mathrm{P}_{\mathrm{u}}} \underline{\mathrm{u}}_{1},
$$

and for a typical stiffener

$$
\underline{\tilde{\mathrm{H}}}=\underline{\mathrm{M}}^{\mathrm{S}} \underline{\mathrm{u}}_{1} .
$$

Here

$$
\begin{aligned}
& \underline{\tilde{n}}=\left(\tilde{n}_{x x^{\prime}}, \tilde{n}_{\theta \theta}, 2 \tilde{n}_{x \theta}\right)^{T}, \\
& \underline{\tilde{H}}=\left(\tilde{\mathrm{H}}_{x x^{\prime}}, \tilde{\mathrm{H}}_{Y Y^{\prime}}, 2 \tilde{\mathrm{H}}_{x y}\right)^{T}, \\
& \underline{u}_{1}=\left(u_{1}, v_{1}, w_{1}\right)^{T}
\end{aligned}
$$

and 


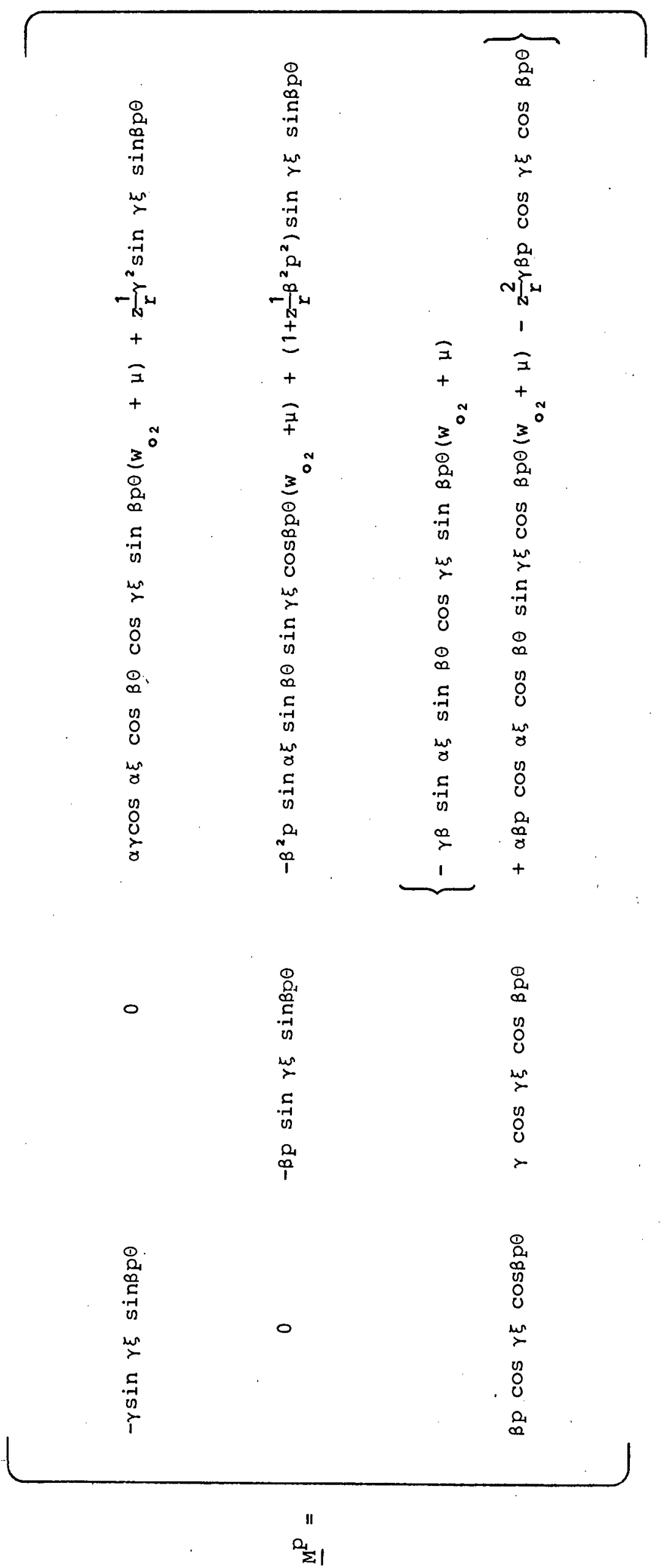




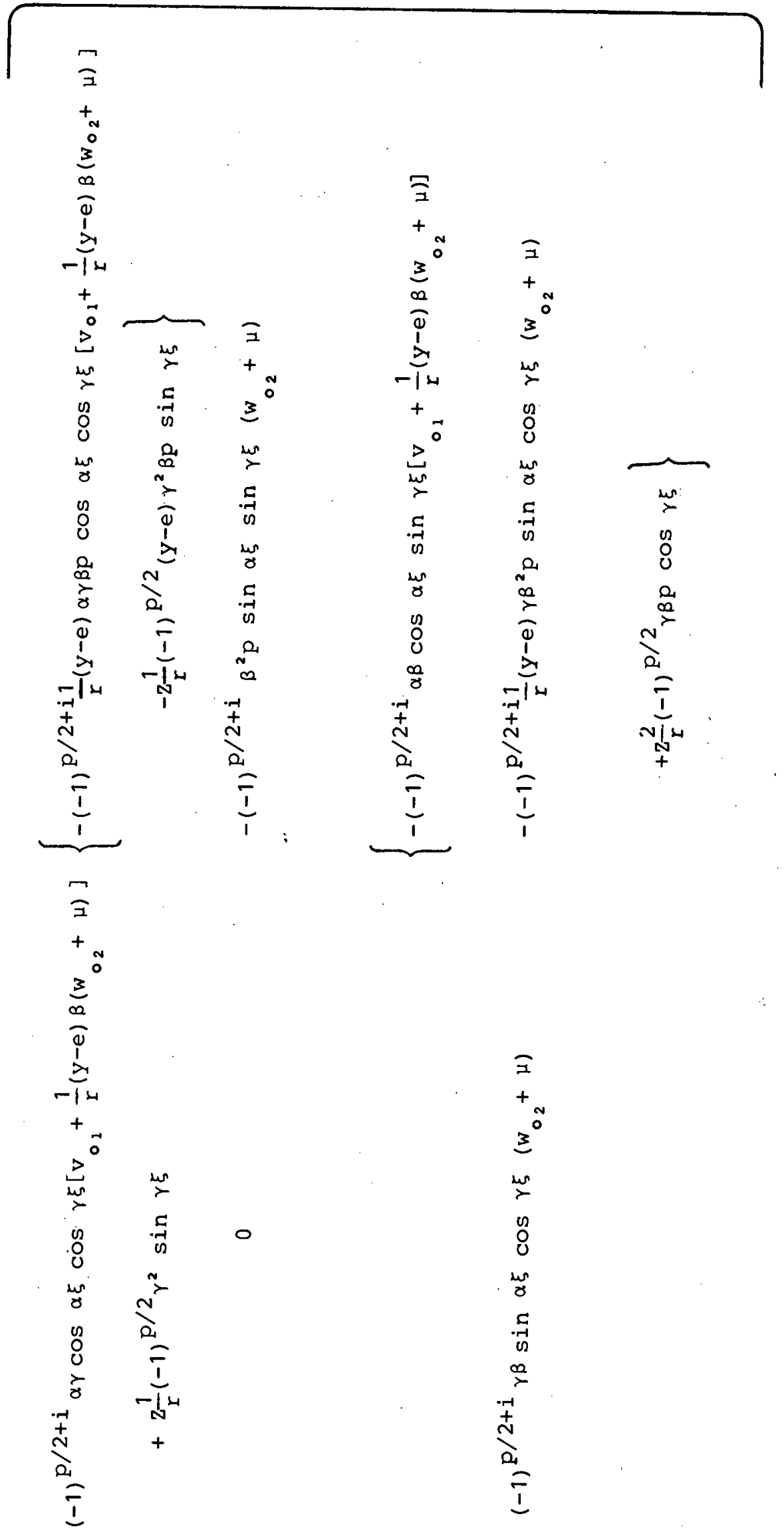

o

o

0 


\subsubsection{Stresses and Stress Resultants}

The comparison stress differences for the panels and stiffeners are obtained using equations (5.17) and (5.18) so that for a typical panel and stiffener we have

$$
\begin{aligned}
& \underline{\tilde{n}}=\underline{\underline{p}}^{-1} \tilde{\sigma}^{p}, \\
& \underline{\mathrm{H}}=\underline{\mathrm{s}}^{-1} \underline{\tilde{\sigma}}^{\mathrm{S}} .
\end{aligned}
$$

where

$$
\begin{aligned}
& \underline{\tilde{\sigma}}^{p}=\left(\tilde{\sigma}_{x x}, \tilde{\sigma}_{\theta \Theta}, \tilde{\sigma}_{x \Theta}\right)^{T}, \\
& \underline{\tilde{\sigma}}^{s}=\left(\tilde{\sigma}_{x x}, \tilde{\sigma}_{Y y}, \tilde{\sigma}_{X y}\right)^{T}
\end{aligned}
$$

and $\underline{\mathrm{P}}^{-1}, \underline{\mathrm{s}}^{-1}$ are defined in Section 5.2.2.

The differences in the membrane stress and bending moment resultants can now be written as follows: for a typical panel

$$
\underline{\tilde{n}}=\int_{-t_{p} / 2}^{t p / 2} \underline{D}^{p} \underline{\sigma}^{p} d z,
$$

and for a typical panel

$$
\tilde{N}=\int_{-t}^{t} \underline{D}_{s / 2}^{s} \underline{\sigma}^{s} d z \text {, }
$$

where $\underline{D}^{p}, \underline{D}^{\mathrm{S}}$ are defined in Section $3 \cdot 5$ and

$$
\begin{aligned}
& \underline{\tilde{n}}=\left(\tilde{n}_{x x}, \tilde{n}_{\theta \Theta}, \tilde{n}_{x \Theta}, \tilde{m}_{x x}, \tilde{m}_{\theta \Theta}, \tilde{m}_{x \Theta}\right)^{T}, \\
& \underline{\tilde{N}}=\left(\tilde{N}_{x x}, \tilde{N}_{y y}, \tilde{N}_{x y}, \tilde{M}_{x x}, \tilde{M}_{y y}, \tilde{M}_{x y}\right)^{T} .
\end{aligned}
$$




\subsubsection{Bifurcation Functional}

It is possible now to obtain the bifurcation functional (5.25) for the complete problem. The variational quantities reduce to variations of the three bifurcation parameters ( ${ }_{1}, v_{1} v_{1}, w_{1}$ ) since the variations of the displacement increments for the $i^{\text {th }}$ panel produce

$$
\begin{aligned}
& \delta \tilde{u}^{(i)}=\delta u_{1}^{(i)} \cos \gamma \xi \sin \beta p \theta^{(i)}, \\
& \delta \tilde{v}^{(i)}=\delta v_{1}^{(i)} \sin \gamma \xi \cos \beta p \theta^{(i),} \\
& \delta \tilde{w}^{(i)}=\delta w_{1}^{(i)} \sin \gamma \xi \sin \beta p \theta^{(i)},
\end{aligned}
$$

and those for the $i^{\text {th }}$ stiffener produce

$$
\begin{aligned}
& \delta \tilde{U}^{(i)}=0, \\
& \delta \tilde{\mathrm{V}}^{(i)}=0, \\
& \left.\delta \tilde{\mathrm{W}}^{(i)}=(-1)^{\mathrm{p} / 2 \mathrm{Lv}_{1}^{(i)}}-\frac{1}{\mathrm{r}}(\mathrm{y}-\mathrm{e}) \beta \mathrm{p} \delta \mathrm{w}_{1}^{(i)}\right] \sin \gamma \xi .
\end{aligned}
$$

The variations of the membrane and bending strain differences as given in Appendix $5 \mathrm{~B}$ for the panels and stiffeners are used with equations (5.36) and (5.37) to obtain the following form of the bifurcation functional:

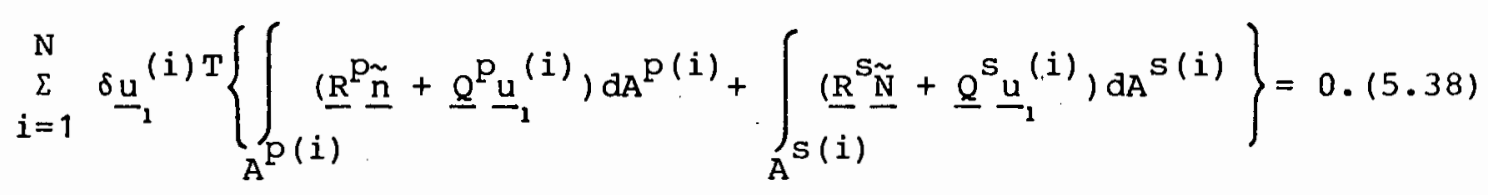

This equation is written out fully in Appendix 5D and for an elastic material (strain hardening exponent, $\mathrm{n}=1$ ) and for $\mathrm{p}=2$ reduces to the equation used by Syngellakis and Walker (1978b). 
Here,

$$
\begin{aligned}
& \underline{u}_{1}{ }^{(i)}=\left(\delta u_{1}^{(i)}, \delta v_{1}^{(i)}, \delta_{1}^{(i)}\right)^{T}, \\
& \underline{u}_{1}^{(i)}=\left(u_{1}^{(i)}, v_{1}^{(i)}, w_{1}^{(i)}\right)^{T},
\end{aligned}
$$

$\underline{\tilde{n}}$ amd $\underline{\tilde{N}}$ have already been defined and 


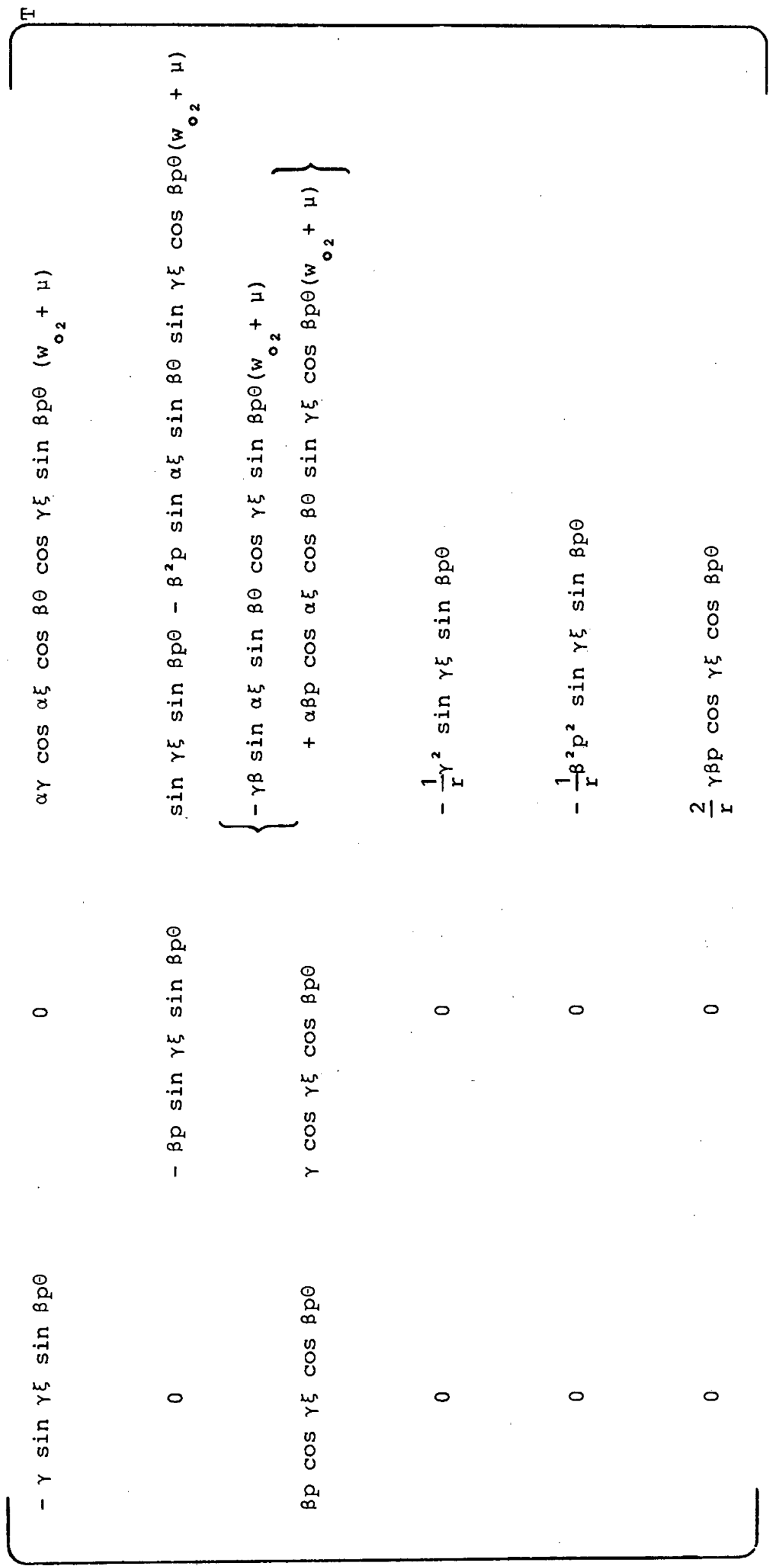




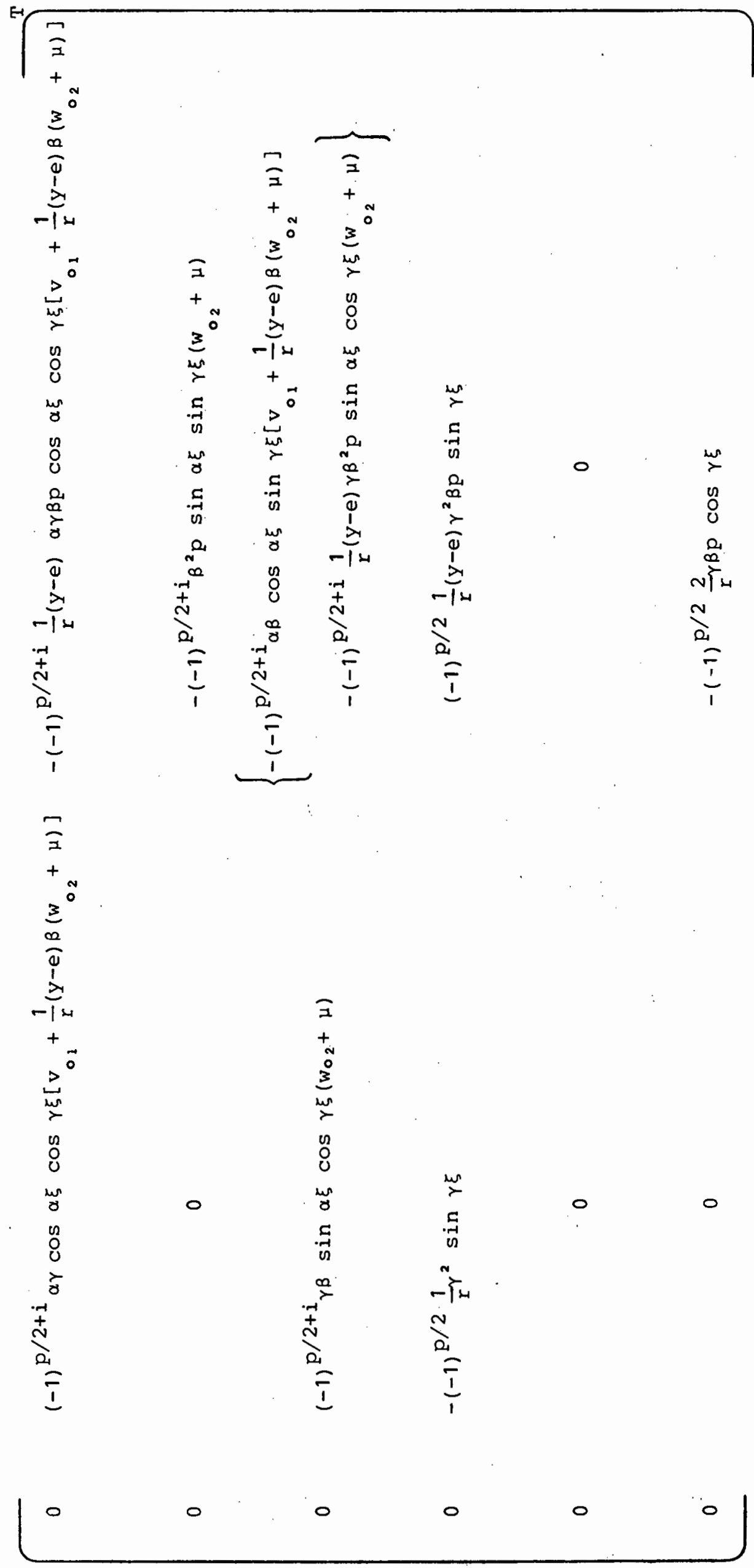




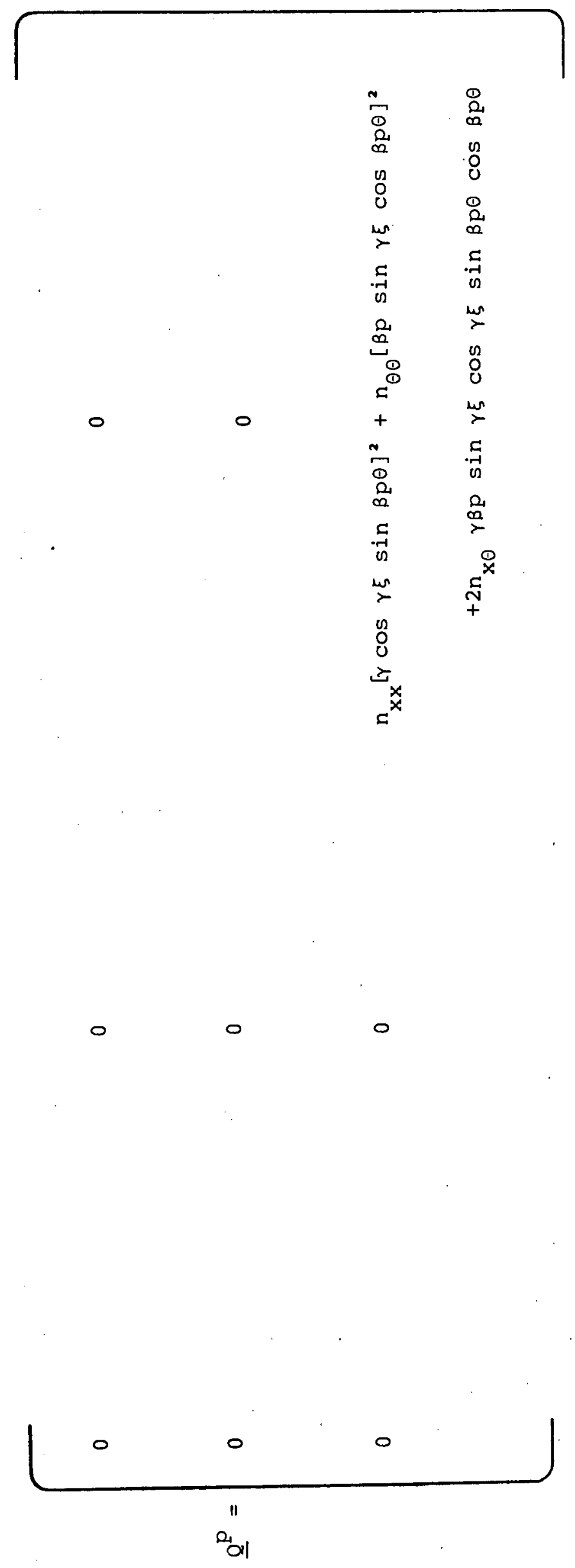




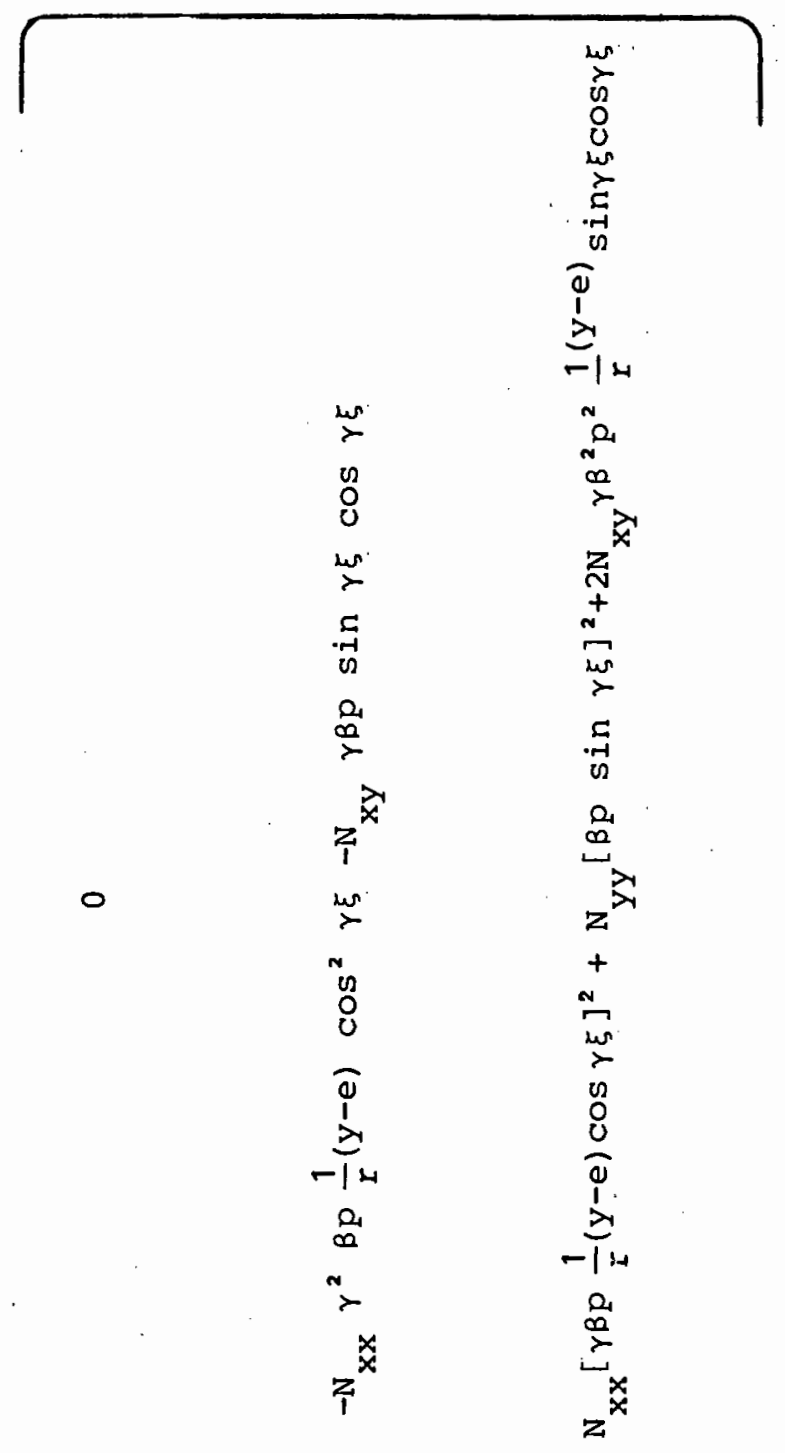

के

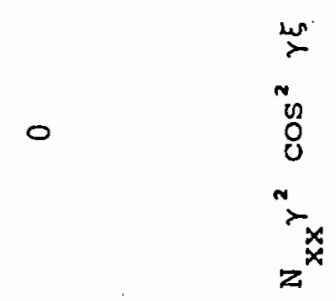


The complete bifurcation problem for a stiffened cylinder has been outlined but the bifurcation functional (5.38) is not in its proper form for us to solve the eigenvalue problem. However, by using the matrix equations of this chapter $(5.30)$ to 5.35$)$ we can write equation (5.38) in the following form:

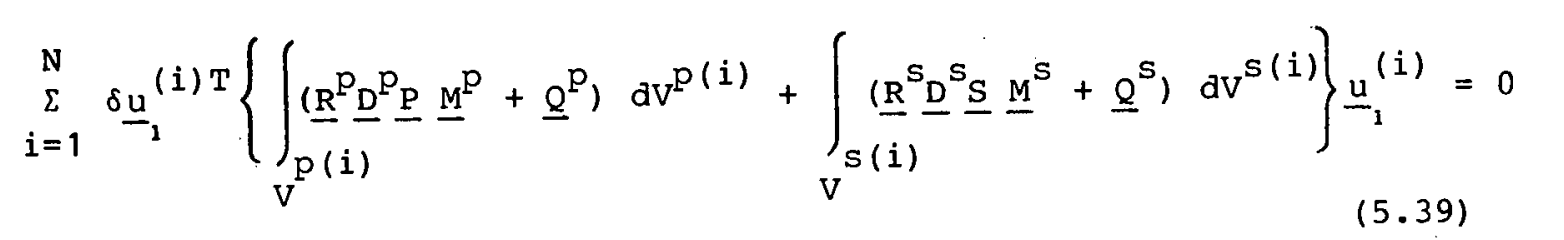

Then the lowest eigenvalue, or bifurcation load, is found when

$$
\left.\left.\operatorname{det}\left[\sum_{i=1}^{N}\left\{\int_{V}^{p(i)} \underline{(R}^{p} \underline{D}^{p} \underline{\underline{p}} \underline{M}^{p}+\underline{Q}^{p}\right) d v^{p(i)}+\int_{V} \underline{R}^{S} \underline{D}^{S} \underline{S} \underline{M}^{s}+\underline{Q}^{s}\right) d v^{S(i)}\right\}\right]=0
$$

where $\operatorname{det}[\ldots]$ means the determinant of $[\ldots]$.

Therefore, at each stage of the load program the value of this determinant is computed and the bifurcation load obtained when it attains its zero value. The proceedures used to obtain the value of this determinant are described in Chapter 6 . 
APPENDIX 5A Derivation of Stiffener Bifurcation Modes.

The bifurcation modes for the $i^{\text {th }}$ panel are those displacement differences in equations (5.26). Using the continuity conditions (3.6) we can obtain the corresponding displacement differences for the $i^{\text {th }}$ stiffener as follows:

$$
\begin{aligned}
& \tilde{u}^{(i)}=\left[u_{1}^{(i)}-w_{1}^{(i)} \frac{1}{r}(y-e) \gamma\right] \cos \gamma \xi \sin \beta p \theta_{0}^{(i)} \\
& \tilde{V}^{(i)}=\zeta w_{1}^{(i)} \sin \gamma \xi \sin \beta p \theta_{0}^{(i)} \\
& \tilde{W}^{(i)}=\left[v_{1}^{(i)}-w_{1}^{(i)} \frac{1}{r}(y-e) \beta p\right] \sin \gamma \xi \cos \beta p \theta_{0}^{(i)} .
\end{aligned}
$$

Since $\beta \theta_{0}^{(i)}=\pi / 2$ for the $i^{\text {th }}$ stiffener,

$$
\begin{aligned}
\sin B p \Theta_{0} & =+1 \text { for } p=1,5,9 \ldots \\
& =-1 \text { for } p=3,7,11 \ldots \\
& =0 \text { for } p \text { even. } \\
\cos B p \Theta_{0} & =-1 \text { for } p=2,6,10 \ldots \\
& =+1 \text { for } p=4,8,12 \ldots \\
& =0 \text { for } p \text { odd. }
\end{aligned}
$$

Now assuming that'p is odd we obtain the following result for the difference in displacement $\tilde{v}^{(i)}$

$$
\tilde{v}^{(i)}= \pm w_{1}^{(i)} \sin \gamma \xi
$$

where the sign depends on the odd value of $p$. This would allow the stiffener to deflect radially as shown in Fig.5A.1 which is highly unlikely. Thus $p$ cannot be an odd integer so that we obtain

$$
\begin{aligned}
& \tilde{\mathrm{U}}^{(i)}=0, \\
& \tilde{\mathrm{v}}^{(i)}=0, \\
& \left.\tilde{\mathrm{W}}^{(i)}=(-1)^{\mathrm{p} / 2}{ }_{1}^{(\mathrm{v})}-\mathrm{w}_{1}^{(i)} \frac{1}{\mathrm{r}}(\mathrm{y}-\mathrm{e}) \beta \mathrm{p}\right] \sin \gamma \xi .
\end{aligned}
$$




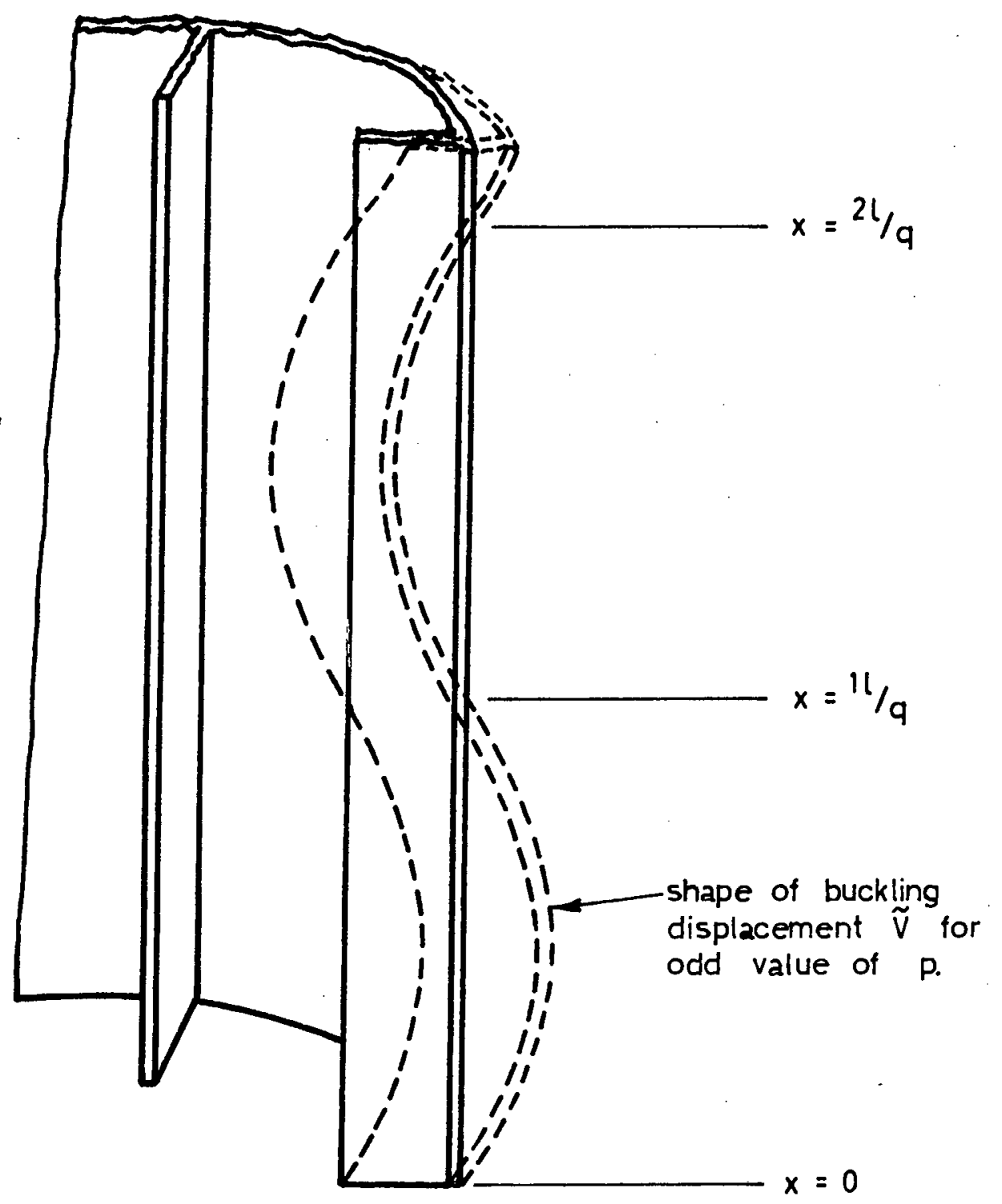

section through cylinder

showing two stiffeners, one

has buckling displacement super-

imposed on it.

Fig. 5A.1 Buckling displacement $\widetilde{V}$ for stiffener when $P$ is odd. 


\section{APPENDIX 5B Midsurface Membrane and Bending Strain Differences}

The midsurface membrane and bending strain differences for a typical panel are

$\left.\tilde{\varepsilon}_{\mathrm{xx}}=-\mathrm{u}_{1} \gamma \sin \gamma \xi \sin \beta p \theta+\mathrm{w}_{1} \alpha \gamma \cos \alpha \xi \cos \gamma \xi \cos \beta \theta \sin \beta p \theta_{\left(\mathrm{o}_{0}\right.}+\mu\right)$, $\tilde{\varepsilon}_{\theta \theta}=-v_{1} \beta p \sin \gamma \xi \sin \beta p \theta+w_{1} \sin \gamma \xi \sin \beta p \theta$

$$
-w_{1} \beta^{2} p \sin \alpha \xi \sin \gamma \xi \sin \beta \theta \cos \beta p \theta\left(w_{0_{2}}+\mu\right) \text {, }
$$

$\tilde{\varepsilon}_{x \Theta}=\frac{1}{2} u_{1} \beta p \cos \gamma \xi \cos \beta p \Theta+\frac{1}{2} v_{1} \gamma \cos \gamma \xi \cos \beta p \theta$

$$
\begin{aligned}
& -\frac{1}{2} w_{1} \gamma \beta \sin \alpha \xi \cos \gamma \xi \sin \beta \theta \sin \beta p \theta\left(w_{0_{2}}+\mu\right) \\
& +\frac{1}{2} w_{1} \alpha \beta p \cos \alpha \xi \sin \gamma \xi \cos \beta \theta \cos \beta p \theta\left(w_{0_{2}}+\mu\right),
\end{aligned}
$$

$\tilde{u}_{x x}=-w_{1} \frac{1}{r} \gamma^{2} \sin \gamma \xi \sin \beta p \theta$,

$\tilde{\mathcal{H}}_{\theta \Theta}=-w_{1} \frac{1}{r} \beta^{2} p^{2} \sin \gamma \xi \sin \beta p \Theta$,

$\tilde{u}_{x \Theta}=w_{1} \frac{1}{r} \gamma \beta p \cos \gamma \xi \cos \beta p \Theta$.

The midsurface membrane and bending strain differences for a typical stiffener are:

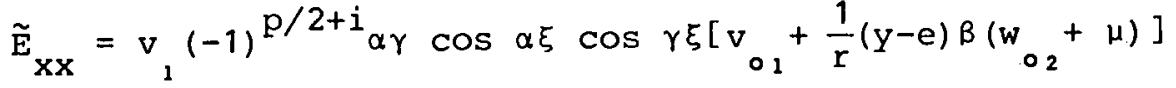

$$
\begin{aligned}
& -w_{1}(-1)^{p / 2+i} \frac{1}{r}(y-e) \alpha \gamma \beta p \cos \alpha \xi \cos \gamma \xi\left[v_{0_{1}}+\frac{1}{r}(y-e) \beta\left(w_{O_{2}}+\mu\right)\right], \\
& \tilde{E}_{Y Y}=-w_{1}(-1)^{p / 2+i} \beta^{2} p \sin \alpha \xi \sin \gamma \xi\left(w_{O_{2}}+\mu\right) \\
& \tilde{E}_{x y}=\frac{1}{2} v_{1}(-1)^{p / 2+i} \gamma \beta \sin \alpha \xi \cos \gamma \xi\left(w_{o_{2}}+\mu\right) \\
& -w_{1}(-1)^{p / 2+i} \alpha \beta \cos \alpha \xi \sin \gamma \xi\left[v_{1}+\frac{1}{r}(y-e) \beta\left(w_{o_{2}}+\mu\right)\right] \\
& -w_{1}(-1)^{p / 2+i} \frac{1}{r}(y-e) \gamma \beta^{2} p \sin \alpha \xi \cos \gamma \xi\left(w_{0_{2}}+\mu\right) \\
& \tilde{\mathrm{k}}_{\mathrm{xx}} / \quad \cdots \cdots
\end{aligned}
$$




$$
\begin{aligned}
& \tilde{\mathrm{K}}_{\mathrm{xx}}=-\mathrm{v}_{1} \frac{1}{\mathrm{r}}(-1)^{\mathrm{p} / 2} \gamma^{2} \sin \gamma \xi+w_{1} \frac{1}{r}(-1)^{\mathrm{p} / 2}(y-e) \gamma^{2} \beta p \sin \gamma \xi, \\
& \tilde{\mathrm{K}}_{y y}=0, \\
& \widetilde{\mathrm{K}}_{\mathrm{xy}}=-w_{2} \frac{1}{\mathrm{r}}(-1)^{\mathrm{p} / 2} \gamma \beta \mathrm{p} \cos \gamma \xi .
\end{aligned}
$$

These midsurface membrane and bending strain differences for a typical panel and stiffener are the same as those used by syngellakis and Walker (1978b).

\section{APPENDIX 5C Bifurcation Equation}

The full bifurcation equation is as follows:

$$
\begin{aligned}
& \sum_{i=1}^{N}\left[\left\{\int_{A}\left[-\tilde{n}_{x x} \gamma \sin \gamma \xi \sin \beta p \theta+\tilde{n}_{x \Theta} \beta p \cos \gamma \xi \cos \beta p \theta\right] d A(i)\right\} \delta u_{1}^{(i)}\right. \\
& +\left\{\int_{A}\left[-n_{\theta \Theta} \beta p \sin \gamma \xi \sin \beta p \Theta+\tilde{n}_{x \Theta} \gamma \cos \gamma \xi \cos \beta p \theta\right] d A p(i)\right. \\
& +\int_{A}\left[\widetilde{N}_{x x}(-1)^{p / 2+i} \alpha \gamma \cos \alpha \xi \cos \gamma \xi\left[v_{0_{1}}^{(i)}+\frac{1}{r}(y-e) \beta\left(w_{o_{2}}+\mu\right)^{(i)}\right]\right. \\
& +\widehat{N}_{x y}(-1)^{p / 2+i} \gamma \beta \sin \alpha \xi \cos \gamma \xi\left(w{ }_{02}+\mu\right)(i) \\
& -\widetilde{M}_{x x}(-1) \mathrm{p} / 2 \frac{1}{r} \gamma^{2} \sin \gamma \xi+N_{x x} v_{1}^{(i)} \gamma^{2} \cos ^{2} \gamma \xi-N_{x x^{w}}{ }^{(i)} \gamma^{2} \beta p \frac{1}{r}(y-e) \cos ^{2} \gamma \xi \\
& \left.\left.-N_{x y}{ }^{(i)} \gamma \beta p \sin \gamma \xi \cos \gamma \xi\right] d A^{s(i)}\right\} \delta v_{1}^{(i)} \\
& +1 \quad \cdots
\end{aligned}
$$


$+\left\{\int_{A}\left[\tilde{n}_{x x} \dot{\alpha} \gamma \cos \alpha \xi \cos \beta \theta \cos \gamma \xi \sin \beta p \theta\left(w_{O_{2}}+\mu\right)^{(i)}+\tilde{n}_{\theta \theta} \sin \gamma \xi \sin \beta p \theta\right.\right.$ $-\tilde{n}_{\theta \theta} \beta^{2} p \sin \alpha \xi \sin \beta \theta \sin \gamma \xi \cos \beta p \theta\left(w_{02}+\mu\right)^{(i)}$ $-\tilde{n}_{x \theta} \gamma \beta \sin \alpha \xi \sin \beta \theta \cos \gamma \xi \sin \beta p \theta\left(w_{02}+\mu\right)^{(i)}$ $+\tilde{n}_{x \Theta} \alpha \beta p \cos \alpha \xi \cos \beta \theta \sin \gamma \xi \cos \beta p \theta\left(w_{o_{2}}+\mu\right)^{(i)}-\tilde{m}_{x x} \frac{1}{r} \gamma^{2} \sin \gamma \xi \sin \beta p \theta$ $-\tilde{m}_{\theta \Theta} \frac{1}{r^{2}} \beta^{2} p^{2} \sin \gamma \xi \sin \beta p \theta+\tilde{m}_{x \theta} \gamma \beta p \cos \gamma \xi \cos \beta p \theta$ $+n_{x x} w_{1}^{(i)}(\gamma \cos \gamma \xi \sin \beta p \theta)^{2}+n_{\theta \theta} w_{1}^{(i)}(\beta p \sin \gamma \xi \cos \beta p \theta)^{2}$ $+2 n_{\left.x \theta_{1}{ }^{(i)} \gamma \beta p \sin \gamma \xi \cos \gamma \xi \sin \beta p \theta \cos \beta p \theta\right] d A} p(i)$ $+\int_{A}\left[-\tilde{N}_{X X}(-1)^{p / 2+i} \frac{1}{r}(y-e) \alpha \gamma \beta p \cos \alpha \xi \cos \gamma \xi\left[v_{o_{1}}^{(i)}+\frac{1}{r}(y-e) \beta\left(w_{o_{2}}+\mu\right)^{(i)}\right]\right.$

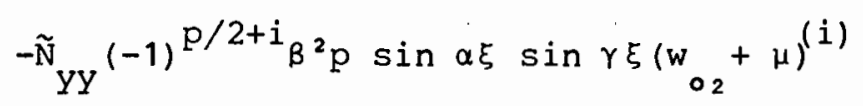
$-\tilde{N}_{x y}(-1)^{p / 2+i} \frac{1}{r}(y-e) \gamma \beta^{2} p \sin \alpha \xi \cos \gamma \xi\left(w_{O_{2}}+\mu\right)^{(i)}$ $-\widetilde{\mathrm{N}}_{\mathrm{xy}}(-1)^{\mathrm{P} / 1+\mathrm{i}} \alpha \beta \cos \alpha \xi \sin \gamma \xi\left[v_{o_{1}}^{(i)}+\frac{1}{\mathrm{r}}(y-e) \beta\left(w_{o_{2}}+\mu\right)^{(i)}\right]$ $+\tilde{M}_{x x}(-1)^{p / 2} \frac{1}{r}(y-e) \gamma^{2} \beta p \sin \gamma \xi-\tilde{M}_{x y}(-1)^{p / 2} \frac{2}{r} \gamma \beta p \cos \gamma \xi$

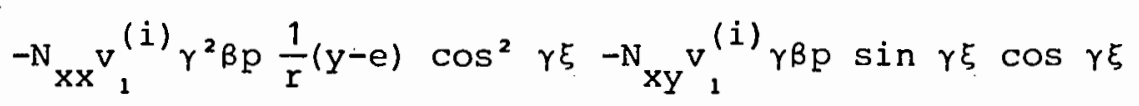
$\left.+\mathrm{N}_{x x{ }_{1}{ }_{1}^{(i)}(\gamma \beta p} \frac{1}{r}(y-e) \cos \gamma \xi\right)^{2}+N_{y y}{ }_{1}{ }_{1}^{(i)}(\beta p \sin \gamma \xi)^{2}$ $\left.\left.\left.+2 N_{x Y_{1}}{ }^{(i)} \gamma \beta^{2} p^{2} \frac{1}{r}(Y-e) \sin \gamma \xi \cos \gamma \xi\right] d A(i)\right\} \delta w_{X}^{(i)}\right]=0$ 


\section{CHAPTER 6 \\ NUMERICAL PROCEDURES FOR BIFURCATION ANALYSIS}

\subsection{Introductory Remarks}

To locate the bifurcation load it is necessary to determine the load at which the value of the determinant, as given in Chapter 5, is zero. This requires the determinant to be evaluated at each solution on the primary path. When the value of the determinant becomes negative the point of bifurcation occurs somewhere between the last two solutions. It is then necessary to iterate as shown in section 6.2 to obtain the exact bifurcation load.

Evaluation of the determinant will require integration through the thicknesses and over the midsurface areas of the panels and stiffeners. This is described in Section 6.3

\subsection{Iteration for bifurcation load: Illinois algorithm}

After each solution (i.e.: values of displacement parameters and external axial stress) has been obtained for a particular value of the loading parameter $u_{0_{1}}$, it is necessary to evaluate the determinant as given in equation (5.40) to determine if the bifurcation load has been obtained. For particular values of $p$, the number of half-waves between two stiffeners in the circumferential direction, and $q$, the number of half-waves in the axial direction, for the bifurcation mode as in equations (5.26) to (5.29), the determinant may take the following values:

$$
\begin{aligned}
\operatorname{det}[\ldots] & >0 \text { if bifurcation load not yet reached, } \\
& =0 \text { if bifurcation occurs at this load, } \\
& <0 \text { if bifurcation occurs before this load. }
\end{aligned}
$$

Thus, when the determinant changes from a positive value at one solution to a negative value at the next solution for particular values of $p$ and $q$, the point of bifurcation lies between these two solutions and can be obtained by iteration.

The iterative method used in this analysis is the Illinois algorithm described by Dahlquist and Björck (1974). This is a modification of the secant method of finding the roots of an equation. Instead of using the two previous values of the function as in the secant method this algorithm uses 
three function values: the most recent one and the two closest points that lie on either side of it. In this analysis the value of the determinant is assumed to be a function of the loading parameter and the procedure used is as follows: suppose that for the $n^{\text {th }}$ solution the loading parameter is given by $x_{n}$ and the value of the determinant as evaluated by equation (5.40) is given by $d_{n}$; this gives the pair $\left(x_{n}, d_{n}\right)$. Then

(i) suppose that at some stage of the loading we have $\left(x_{n-1}, d_{n-1}\right)$ and $\left(x_{n}, d_{n}\right)$ such that $d_{n-1} d_{n}<0$;

(ii) we can therefore obtain $x_{n+1}$ using the secant method (or another method) and thus obtain the pair $\left(x_{n+1}, d_{n+1}\right)$;

(iii) if $d_{n+1} d_{n}<0$ then find $x_{n+2}$ by the secant method as follows

$$
x_{n+2}=x_{n+1}-d_{n+1} \frac{x_{n+1}-x_{n}}{d_{n+1}-d_{n}}
$$

(iv) but if $d_{n+1} d_{n-1}<0$ then find $x_{n+2}$ by the modified secant method as follows:

$$
x_{n+2}=x_{n+1}-d_{n+1} \frac{x_{n+1}-x_{n-1}}{d_{n+1}-\alpha d_{n-1}} \text {, }
$$

$0<\alpha<1$; generally (as in this analysis) $\alpha=\frac{1}{2}$.

The $(n-1)^{\text {th }}$ pair now becomes $\left(x_{n-1}, \alpha d_{n-1}\right)$ :

(v) using $x_{n+2}$ the corresponding $d_{n+2}$ can be obtained;

(vi) using the pair $\left(x_{n+2}, d_{n+2}\right)$ and the closest pairs that lie on either side, the steps (iii) to (v) can be repeated until the value of $x$ does not change, i.e.: the value of the determinant is zero.

This algorithm is presented diagrammatically in Fig. 6.1.

It must be noted that when a new value of load parameter is computed as in step (iii) or (iv), then a new solution for this value of load parameter must be obtained before the determinant is evaluated as in step (v). 


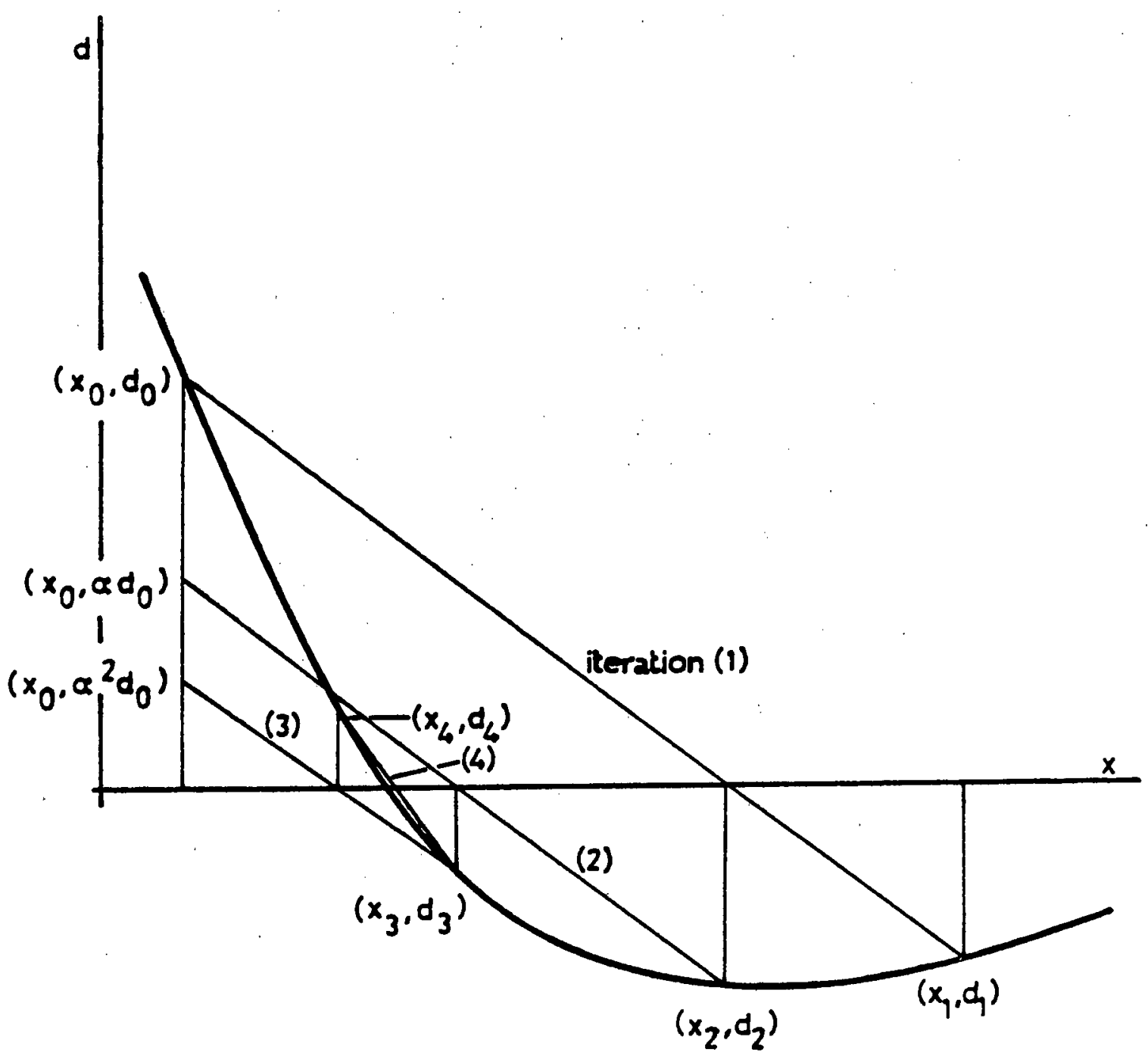

Fig. 6.1 Illinois Algorithm. 


\subsection{Numerical Integration}

\subsubsection{General}

The numerical integration techniques used to evaluate the integrals required for the evaluation of equation (5.40) are the same as those described in Chapter 4 for the primary path. The modified Gaussian Quadrature formulae of Section 4.3.1 are used to evaluate the integrals through the thicknesses of the panels and stiffeners. The formula given in section 4.3 .1 for integration over an area is used to integrate over the midsurface areas of the panels and stiffeners in order to evaluate the determinant as in equation $(5.40)$. However, the limits of the integrations are different from those of the primary path.

\subsubsection{Numerical integration over the midsurface areas}

Integrations over the midsurface areas are required in equation (5.40) which can be written, with abbreviated integrands, as follows

$$
\left.\sum_{i=1}^{N}\left[\int_{A}(i)\right)^{p} d A^{p(i)}+\int_{A}(\ldots)^{s} d A^{s(i)}\right]
$$

It was found that for arbitrary values of the half-wave length parameters $m$, for the primary path, as well as $p$ and $q$, for the bifurcated path, the contributions to these integrals from each pair of adjacent panels and from each stiffener were equal. Thus equation (6.3) can be written as

$$
\beta r\left[\int_{0}^{\ell} \int_{-\theta_{0}}^{\theta_{0}}(\ldots)^{\mathrm{p}} \mathrm{d} \theta \mathrm{dx}+\int_{0}^{\ell} \int_{\theta_{0}}^{3 \theta_{0}}(\ldots)^{\mathrm{p}} \mathrm{d} \theta \mathrm{dx}\right]+\mathrm{N} \int_{0}^{\ell} \int_{-d}^{\mathrm{d}}(\ldots)^{\mathrm{s}} \mathrm{dydx} \cdot(6.4)
$$

If $m$ is even then all panels have the same contributions. There exist no symmetries in the $\dot{\theta}$ and $y$ directions for the panel and stiffener, respectively. For the integrations in the $x$ direction the combination of different values of $q$ for a particular value of $m$ make it necessary to integrate over each of the $m$ half-waves in the axial direction. Thus equation (6.4) 
becomes :

$$
\begin{gathered}
\sum_{j=0}^{(m-1)} r \beta \int_{j \ell / m}^{(j+1) \ell / m} \int_{-\theta_{0}}^{\theta_{0}}(\ldots)^{p} d \theta d x+r \beta \int_{j \ell / m}^{(j+1) \ell / m} \int_{\theta_{0}}^{3 \theta_{0}}(\ldots)^{p} d \theta d x \\
+N \int_{j \ell / m}^{(j+1) \ell / m} \int_{-d}^{d}(\ldots)^{s} d y d x
\end{gathered}
$$

Due to the combination of the values of $\mathrm{m}$ and $\mathrm{q}$ it was necessary to use 12 Gausspoints per half-wave in the axial direction and 10 Gausspoints per panel or stiffener in the $\theta$ or $y$ directions, respectively.

\subsection{Concluding Remarks}

These are the only numerical techniques used in the bifurcation analysis that need discussion, as most of this part of the analysis requires numerical computation only. The procedure for evaluating the determinant and obtaining the bifurcation load is outlined in the program flow chart shown in Fig. 6.2. 


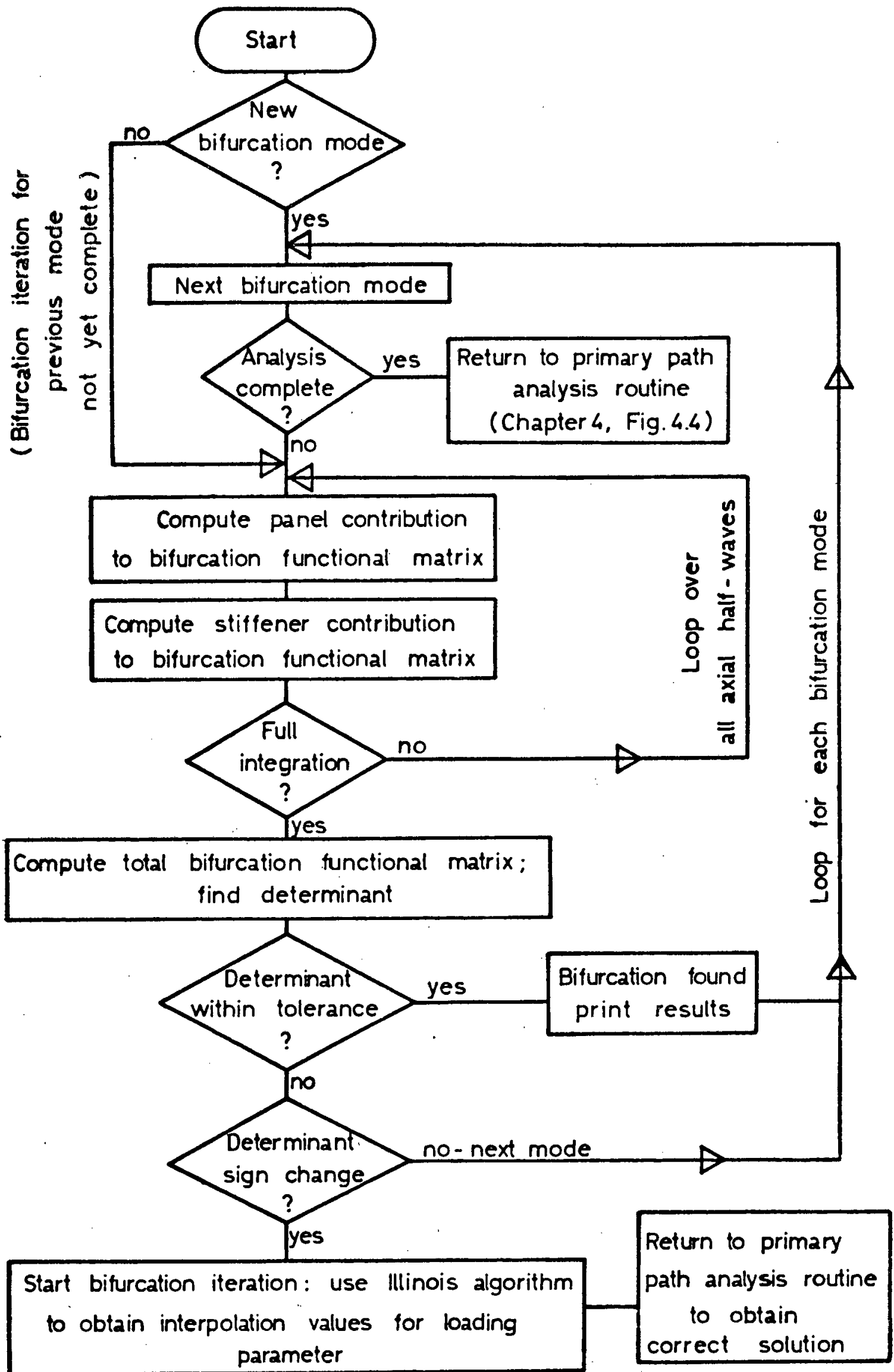

Fig.6.2 Bifurcation Analysis Flow Chart. 
CHAPTER 7

RESULTS AND DISCUSSION

\subsection{Introductory Remarks}

The computer program as described in Chapters 4 and 6 can be used to obtain complete information of the primary equilibrium path and also to locate the points at which bifurcation occurs. The cylinder geometry is arbitrary and therefore it is possible to investigate the effects of all the geometric parameters but since these are numerous this presents a formidable task. Initial imperfections are closely related to the thickness of the panels and thus the effects of different panel thicknesses will be investigated. These effects are looked at with respect to two shell geometries which differ in that they have different stiffener depths. This investigation is presented in Section 7.3, while particular shell geometries are used to compare with previous results and experimental results in sections 7.2 and 7.4 respectively.

\subsection{Comparison with Previous Results}

This analysis is an extension into the plastic range of the work done by Syngellakis and walker (1978b) where they obtained results for an imperfect elastic cylinder. The analysis produced in the previous chapters must be able to reduce to the elastic case as well as to the perfect shell analysis.

For the imperfect cylinder in the elastic range direct comparisons can be made with the results obtained by Syngellakis and Walker (1978b). These comparisons comprise the critical stresses which are shown in the table in Fig 7.1, the external axial stess versus end-shortening curve also shown in Fig. 7.1 and the external axial stress versus displacement parameter $w_{\mathrm{O}_{2}}$ curve shown in Fig. 7.2. It is clearly seen that the approximate numerical analyses used in this analysis compares favourably with the exact analysis of Syngellakis and Walker (1978b), (Note: the value of $\mathrm{w}_{\mathrm{O}_{2}}$ in the load versus displacement parameter diagram, Fig. 6, should be a factor of 10 smaller).

\subsection{Results obtained.}

\subsubsection{Introductory aspects}

All the results presented in this section are for an internally stiffened cylinder/ $\ldots$.... 
Fig. 7.1 Load vs. End shortening curves.

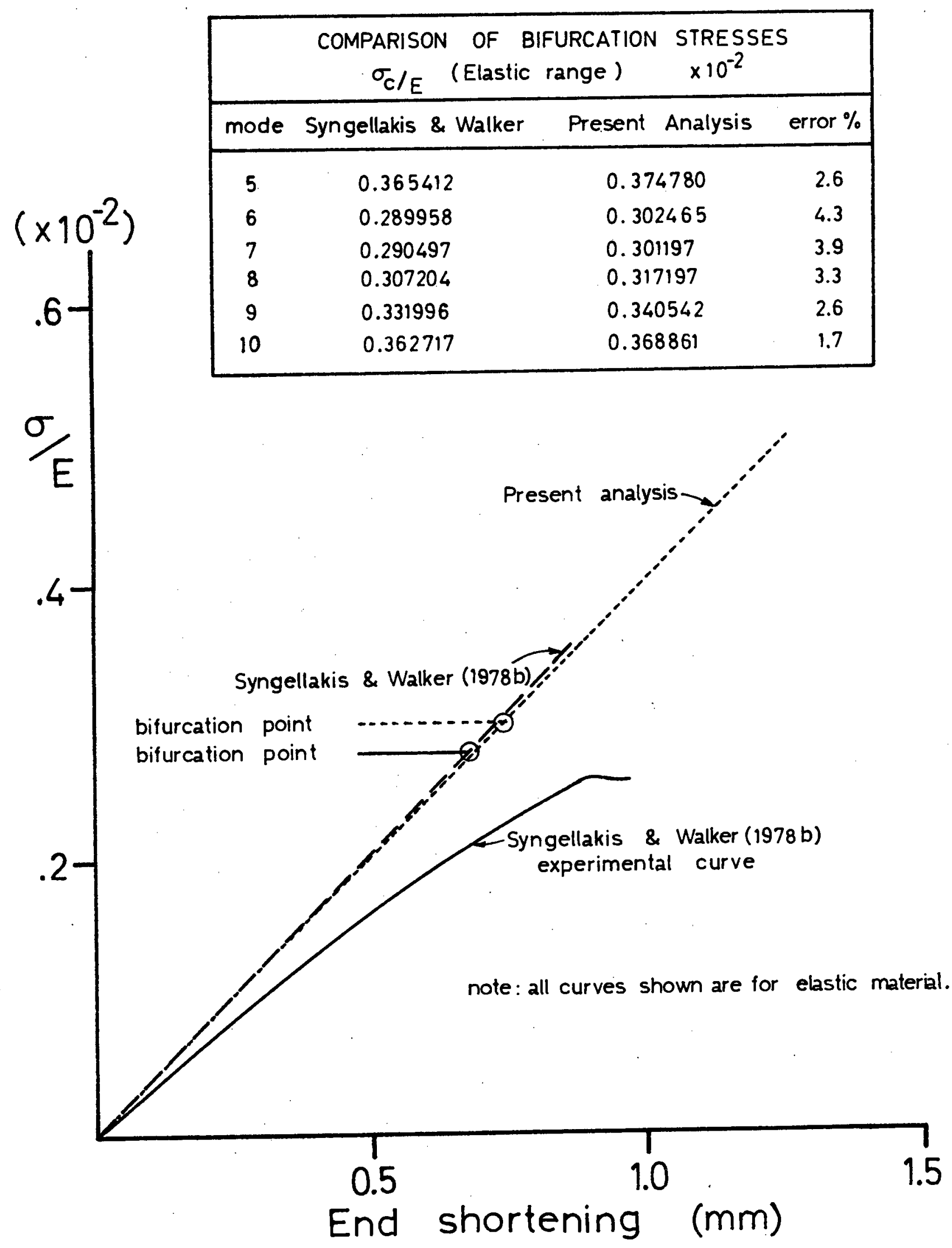


Fig. 7.2 Load vs. $\mathrm{w}_{02}$ curves.

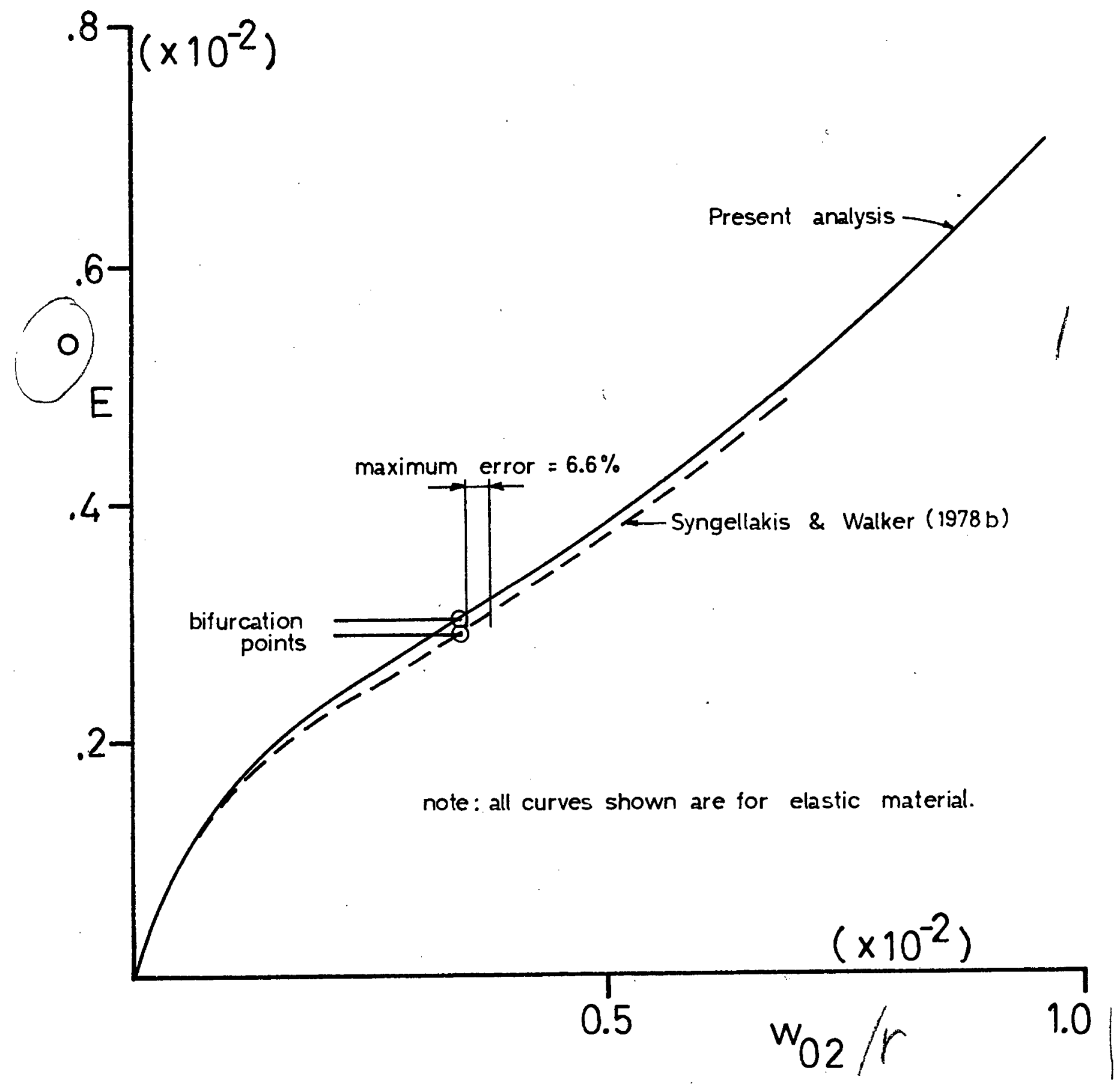


with 10 stiffeners. The shell geometry is described by the following nondimensional parameters: stiffener thickness $t_{S} / r=0,0125$, stiffener depth $d / t_{s}=5$ or 10 and $t_{p} / r=75,100,150$ or 200 . The buckled mode of a perfect cylinder as viewed by syngellakis and walker (1978a) had 2 half-waves in the axial direction and one half wave between adjacent stiffeners in the circumferential direction. This corresponds with the value of $m=2$ which, as with Syngellakis and walker (1978b) shall be used in this analysis; also, $\alpha=2$ is used.

For the material parameters, strain hardening exponents $\mathrm{n}=1$ (elastic), 3 (high strain hardening) or 10 (low strain hardening) shall be used; the yield stress in simple tension is given by the non-dimensional parameters $\sigma_{y} / E=0,002$. The bifurcation modes to be considered have an arbitrary number of half-waves in the axial direction and 2 half-waves between adjacent stiffeners in the circumferential direction, thus being antisymmetric with respect to the panel mid-generator. Bifurcation modes with more half-waves between adjacent stiffeners yield higher critical stresses and are therefore not considered here. The external axial stress at bifurcation, the critical stress, is given by the non-dimensional parameter $\sigma_{C} / \mathrm{E}$.

\subsubsection{Imperfection Sensitivity}

It is well known that elastic unstiffened imperfect cylinders reach a limit point or maximum load at a lower value than the bifurcation stress of a perfect cylinder after which their deformation behaviour approaches the postbuckling behaviour of the perfect cylinder. Tvergaard (1977) showed that the behaviour of an imperfect cylindrical panel also approaches the postbuckling behaviour of the perfect panel in the plastic range but that under certain conditions a maximum is not obtained. These trends are to be investigated for a stiffened cylinder in the plastic range.

It is not possible to obtain the post-bifurcation behaviour of a perfect stiffened cylinder using the procedures set out in previous chapters but it can be assumed that as the size of the imperfection decreases the behaviour of the imperfect shell will converge to the post-bifurcation behaviour of the perfect shell. Thus, as with Tvergaard (1977), the post-bifurcation behaviour of a perfect shell is computed using a very small inperfection. In 


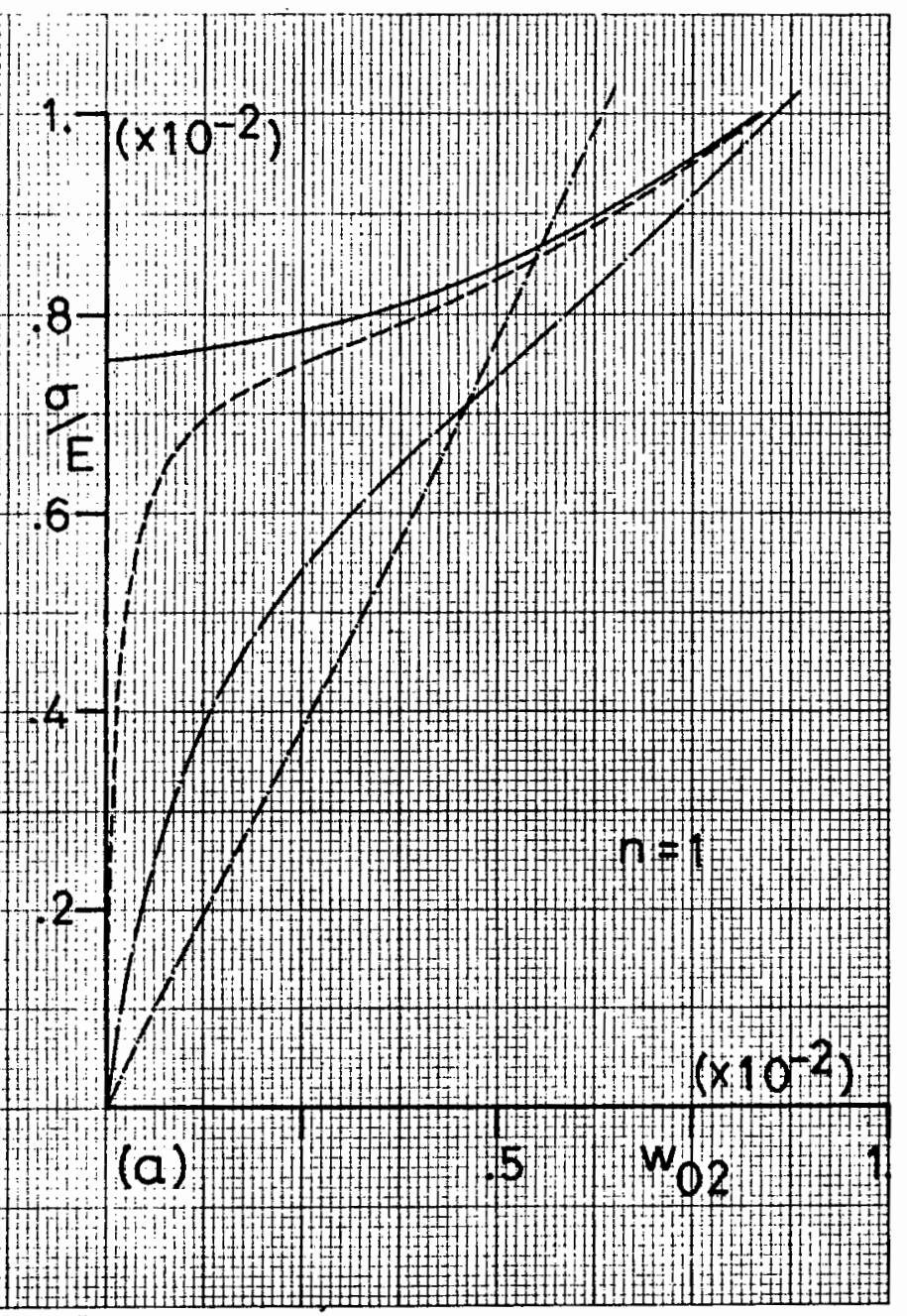

$$
\begin{aligned}
\text { Fig. } 7.3 \quad & d t_{s}=5 \\
r / t_{p} & =75
\end{aligned}
$$

\begin{tabular}{|c|c|c|c|}
\hline \multicolumn{2}{|c|}{$\sigma_{\mathrm{c}} / \mathrm{E}$} & \multicolumn{2}{|c|}{$\left(\times 10^{-2}\right)$} \\
\hline$\mu_{t_{p}}{ }^{n}$ & 1 & 3 & 10 \\
\hline 0 & 0.834 & 0.429 & 0.278 \\
\hline .01 & 0.898 & 0.451 & 0.283 \\
\hline .10 & 0.957 & 0.455 & 0.283 \\
\hline 1.0 & 1.336 & 0.496 & 0.289 \\
\hline & $\begin{aligned} \text { perfec } & \\
P & =0.0 \\
& =0.1 \\
& =1.0\end{aligned}$ & & $\bar{c}$ \\
\hline & .75 & .38 & .26 \\
\hline
\end{tabular}

Bifurcation stresses
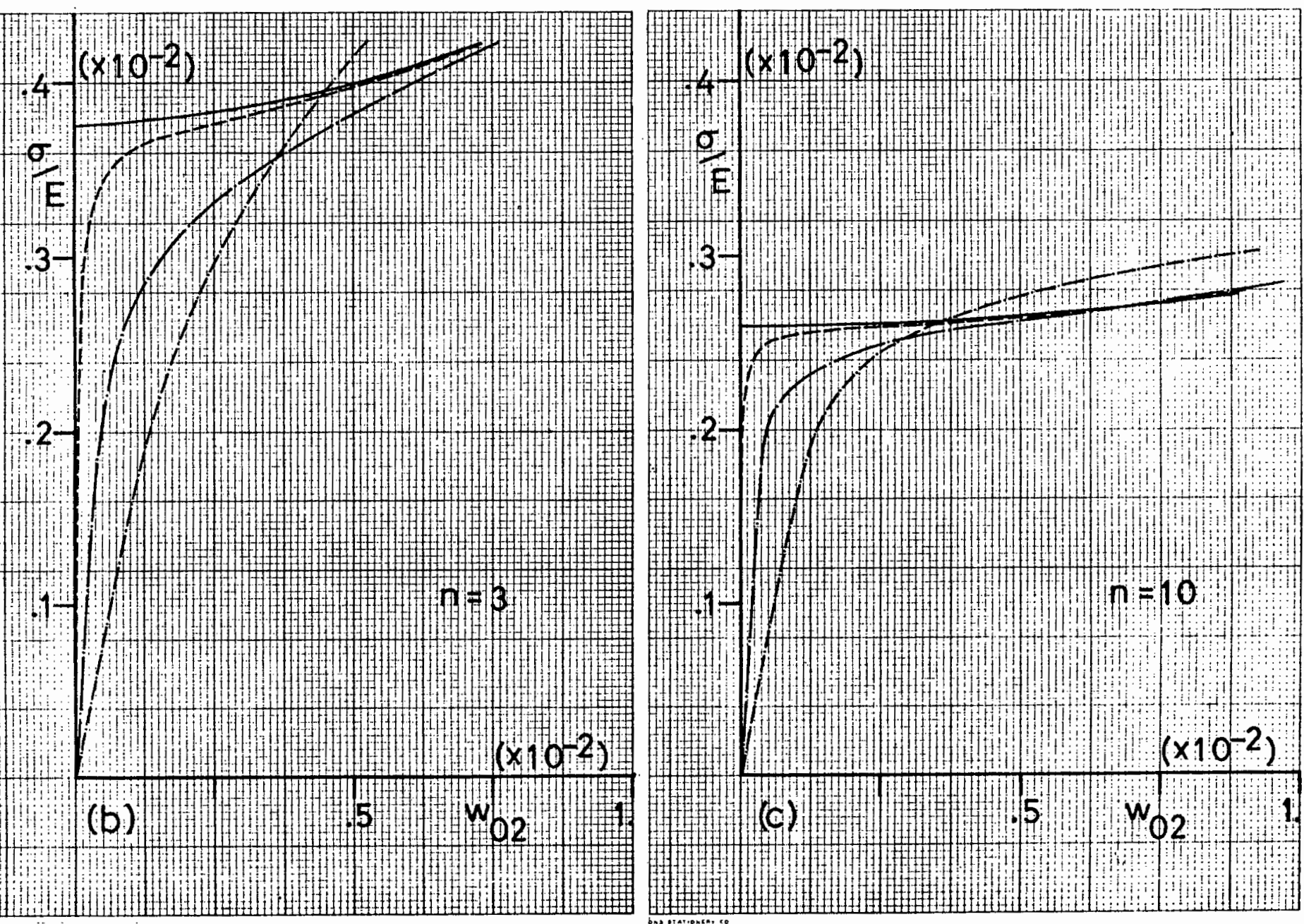


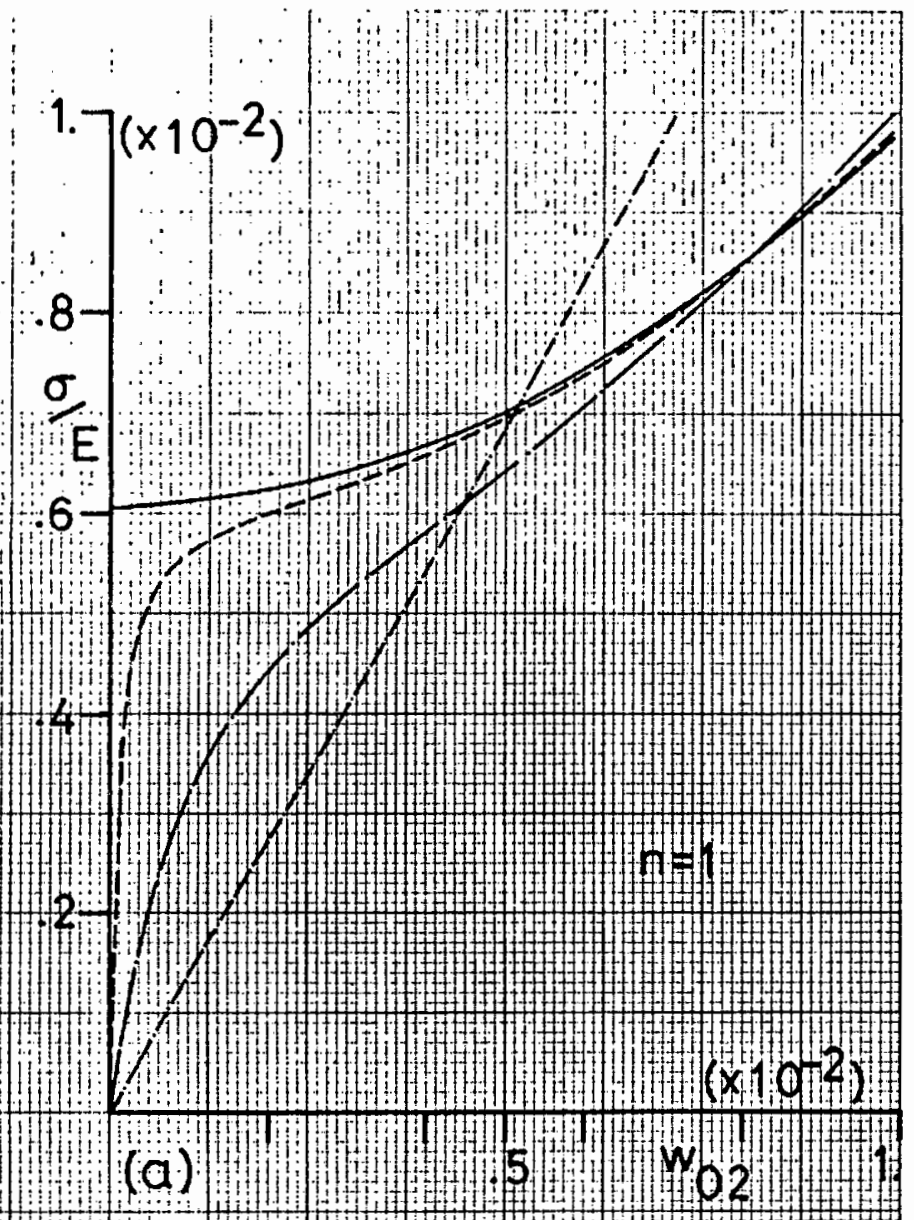

$\begin{aligned} \text { Fig. } 7.4 \quad & d t_{t_{s}}=5, \\ r / t_{p} & =100\end{aligned}$

Bifurcation stresses

\begin{tabular}{|c|c|c|c|}
\hline \multicolumn{2}{|r|}{$\sigma_{c} / E$} & \multicolumn{2}{|c|}{$\left(\times 10^{-2}\right)$} \\
\hline & .1 & 3 & 10 \\
\hline 0 & 0.582 & 0.365 & 10.262 \\
\hline & & | 0.389 & \\
\hline
\end{tabular}

$\begin{array}{lllll}.10 & 0.647 & 0.392 & 0.269\end{array}$

\begin{tabular}{ll|l|l|l|}
1.0 & 0.900 & 0.426 & 0.275 \\
\hline
\end{tabular}
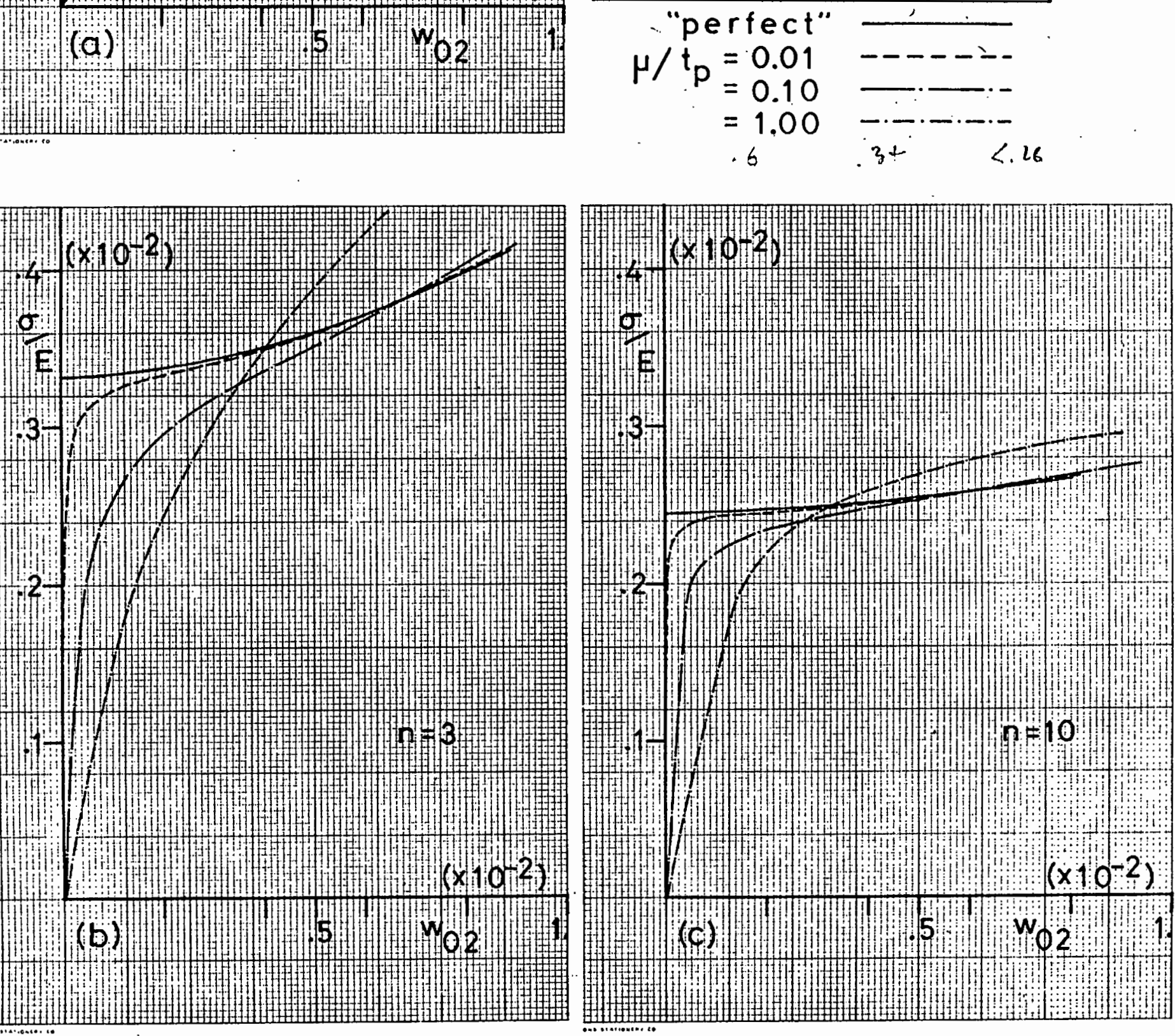


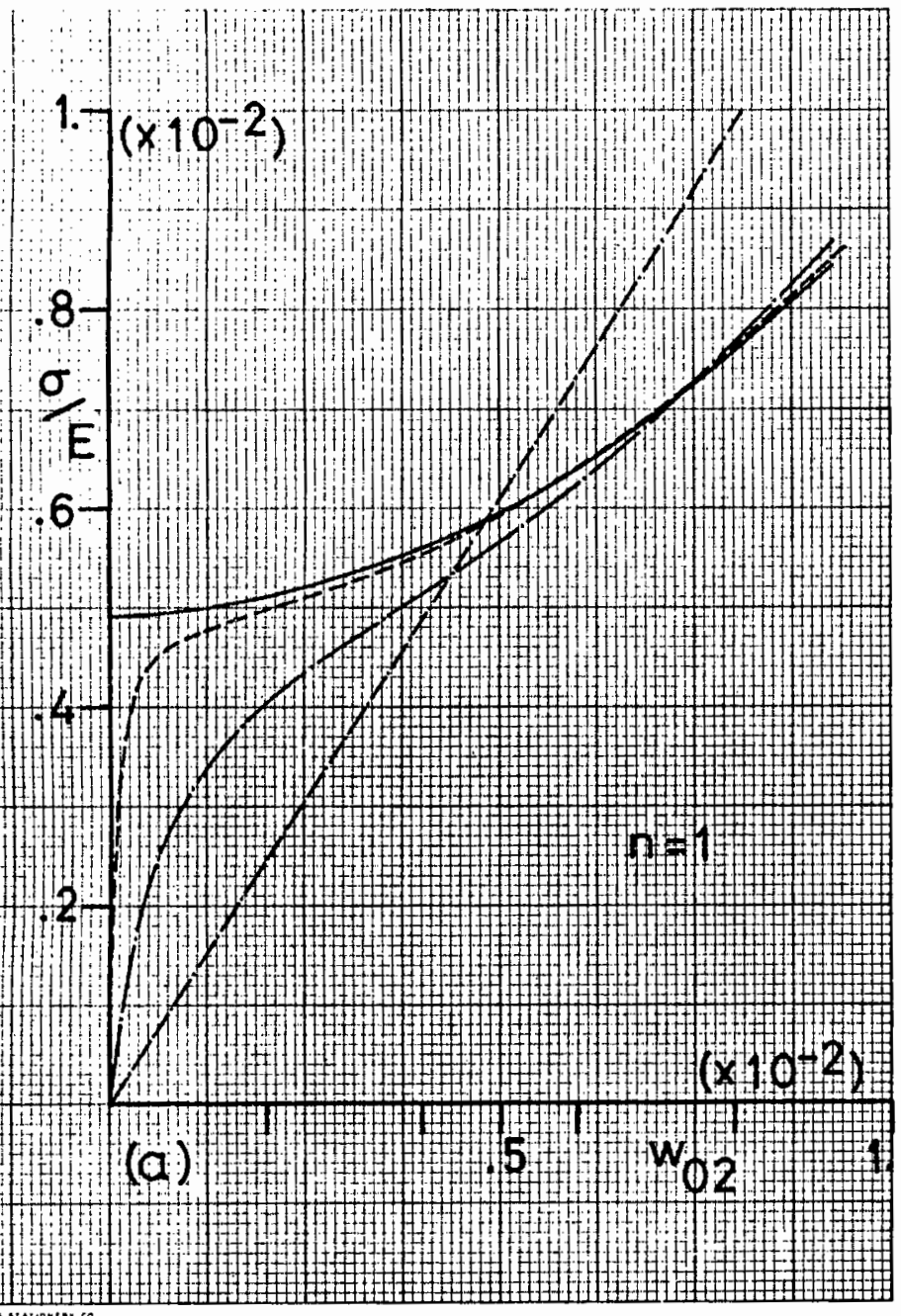

Fig. $\begin{aligned} 7.5 \quad & d t_{t_{s}}=5 \\ r / t_{p} & =150\end{aligned}$

Bifurcation stresses

\begin{tabular}{|c|c|c|c|}
\multicolumn{3}{|c|}{$\sigma_{c} / E$} & \multicolumn{1}{c|}{$\left(\times 10^{-2}\right)$} \\
\hline$\mu_{t_{p}}$ & 1 & 3 & 10 \\
\hline 0 & 0.397 & 0.300 & 0.242 \\
\hline 01 & 0.397 & 0.328 & 0.259 \\
\hline
\end{tabular}

\begin{tabular}{|l|l|l|l|}
\hline .01 & 0.397 & 0.328 & 0.259 \\
\hline .10 & 0.434 & 0.341 & 0.260 \\
\hline
\end{tabular}

\begin{tabular}{|l|l|l|l|}
\hline 1.0 & 0.623 & 0.367 & 0.261 \\
\hline
\end{tabular}

$$
\begin{aligned}
\mu / t_{p} & =0.01 \\
& =0.10 \\
& =1.00
\end{aligned}
$$
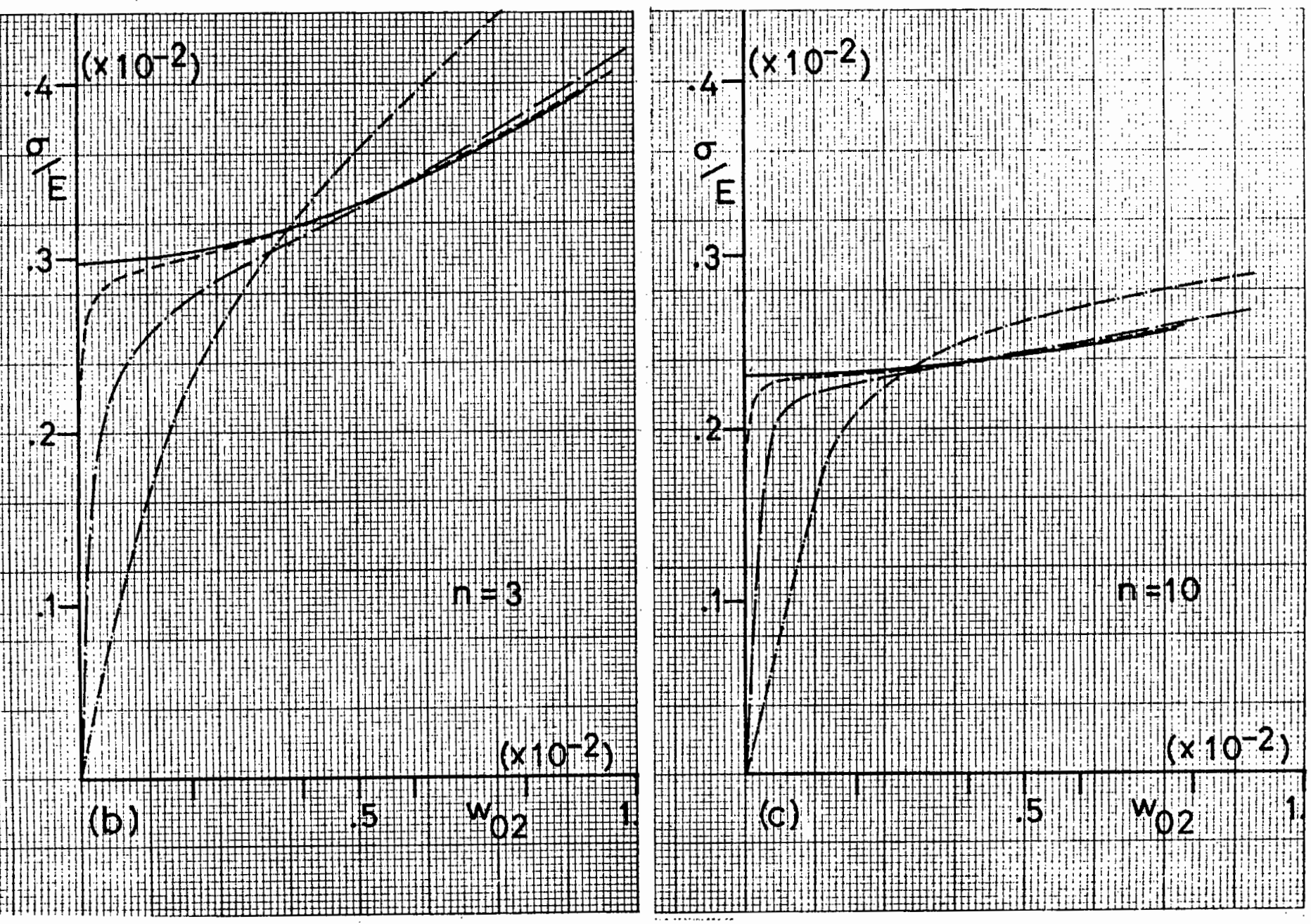


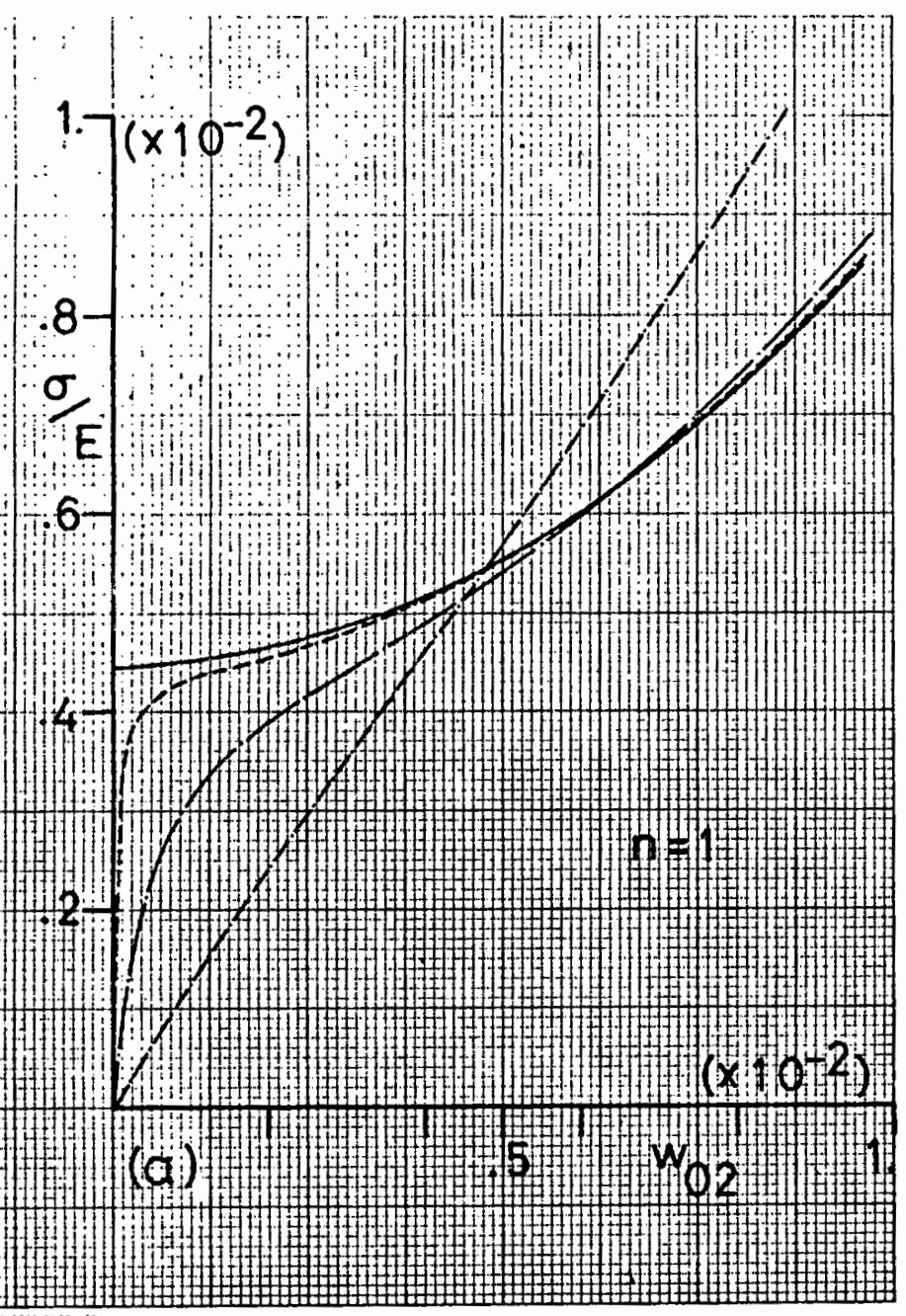

$$
\text { Fig. } 7.6 \quad \begin{aligned}
d_{t_{s}} & =5 \\
r t_{p} & =200
\end{aligned}
$$

Bifurcation stresses $\sigma_{c} / E \quad\left(\times 10^{-2}\right)$ \begin{tabular}{|c|c|c|c|}
\hline$\mu / t_{p}$ & 1. & 3 & 10 \\
\hline 0 & 0.324 & 0.269 & 0.231 \\
\hline .01 & 0.324 & 0.269 & 0.253 \\
\hline
\end{tabular}

\begin{tabular}{|l|l|l|l|}
\hline .01 & 0.324 & 0.269 & 0.253 \\
\hline .10 & 0.343 & 0.316 & 0.254 \\
\hline 1.0 & 0.544 & 0.348 & 0.257 \\
\hline
\end{tabular}

$$
\begin{aligned}
& \text { "perfect" } \\
& \mu / t_{p}=0.01 \\
& 0.10 \\
&=0 \\
& 1.00
\end{aligned}
$$
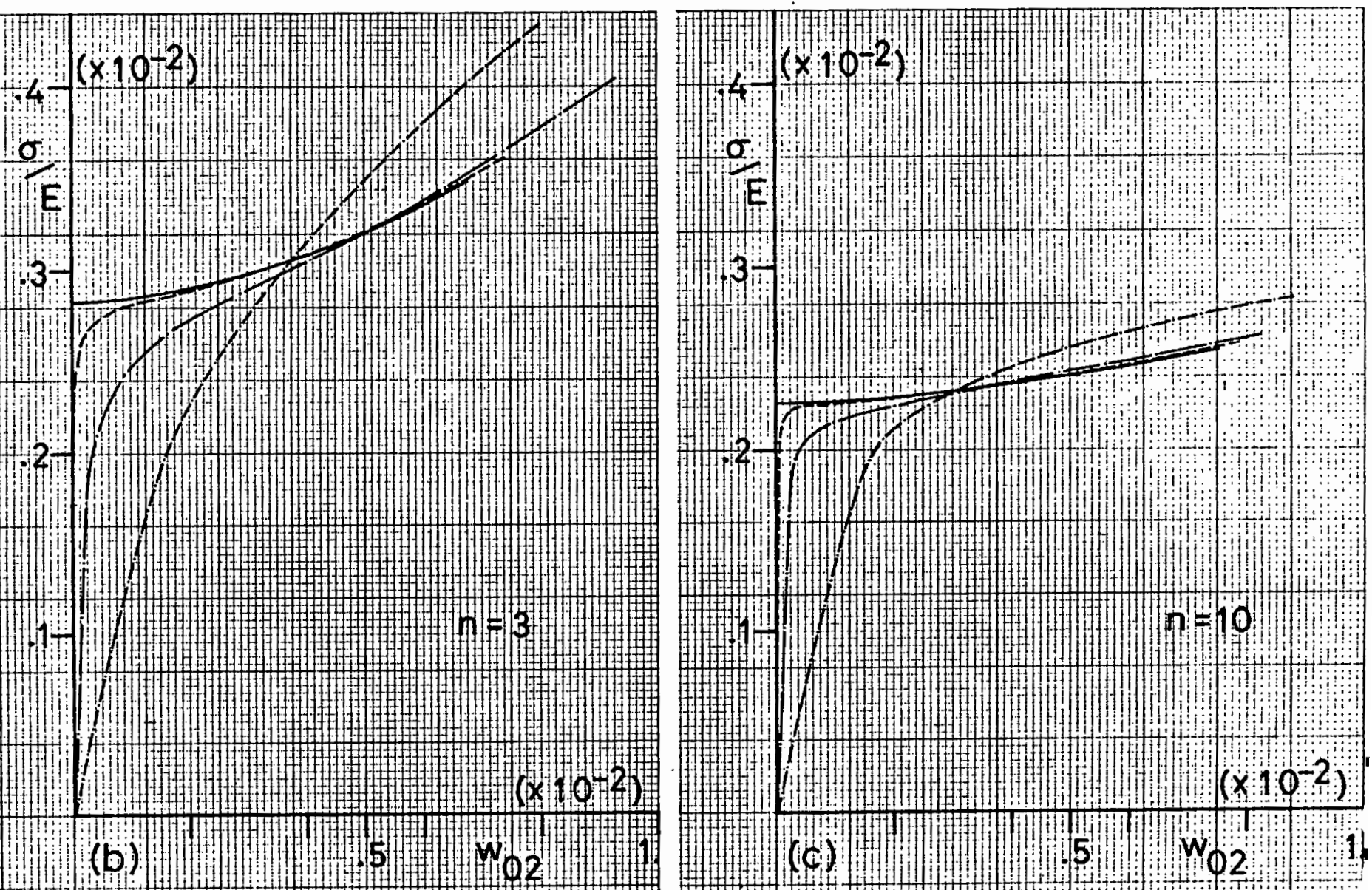


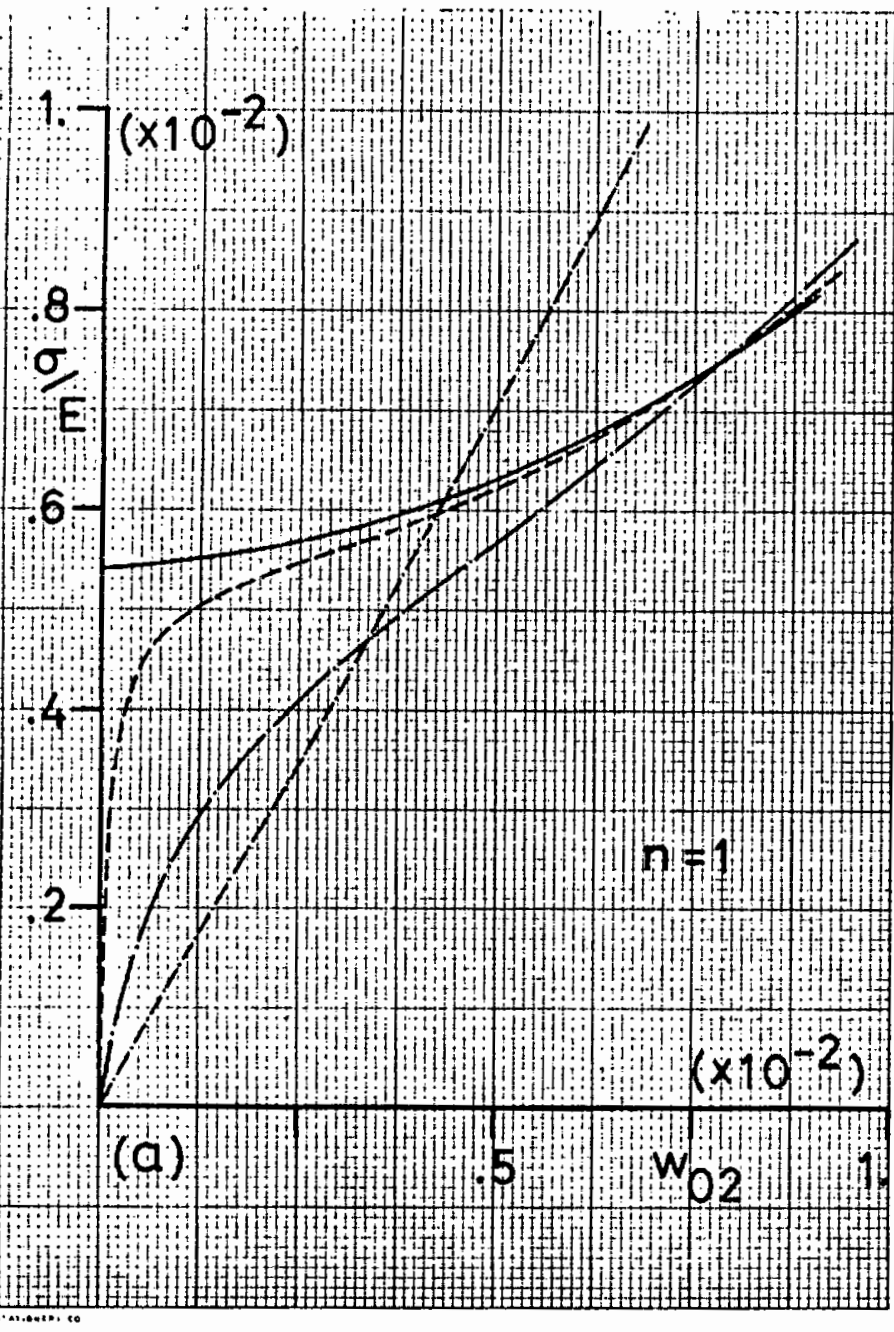

$$
\text { Fig. } \begin{aligned}
7.7 \quad & d / t_{s}=10 \\
r / t_{p} & =75
\end{aligned}
$$

Bifurcation stresses

\begin{tabular}{|c|c|c|c|}
\multicolumn{3}{|c|}{$\sigma_{c} / E$} & $\left(\times 10^{-2}\right)$ \\
\hline$\mu / t_{p}^{n}$ & 1 & 3 & 10 \\
\hline 0 & 0.468 & 0.328 & 0.252 \\
\hline .01 & 0.469 & 0.335 & 0.258 \\
\hline .10 & 0.524 & 0.354 & 0.261 \\
\hline 1.0 & 0.991 & 0.437 & 0.278 \\
\hline
\end{tabular}

$$
\text { "perfect" }
$$

$$
\begin{aligned}
\mu / t_{p} & =0.01 \\
& =0.10 \\
& =1.00
\end{aligned}
$$

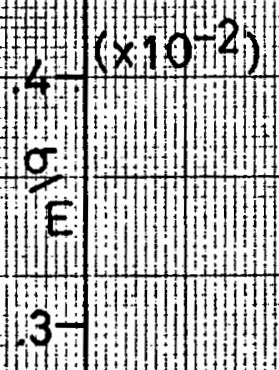

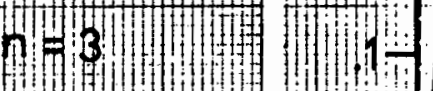

$n=10$

$(x) 0=$

$\sigma$

3.

$2=(1)$
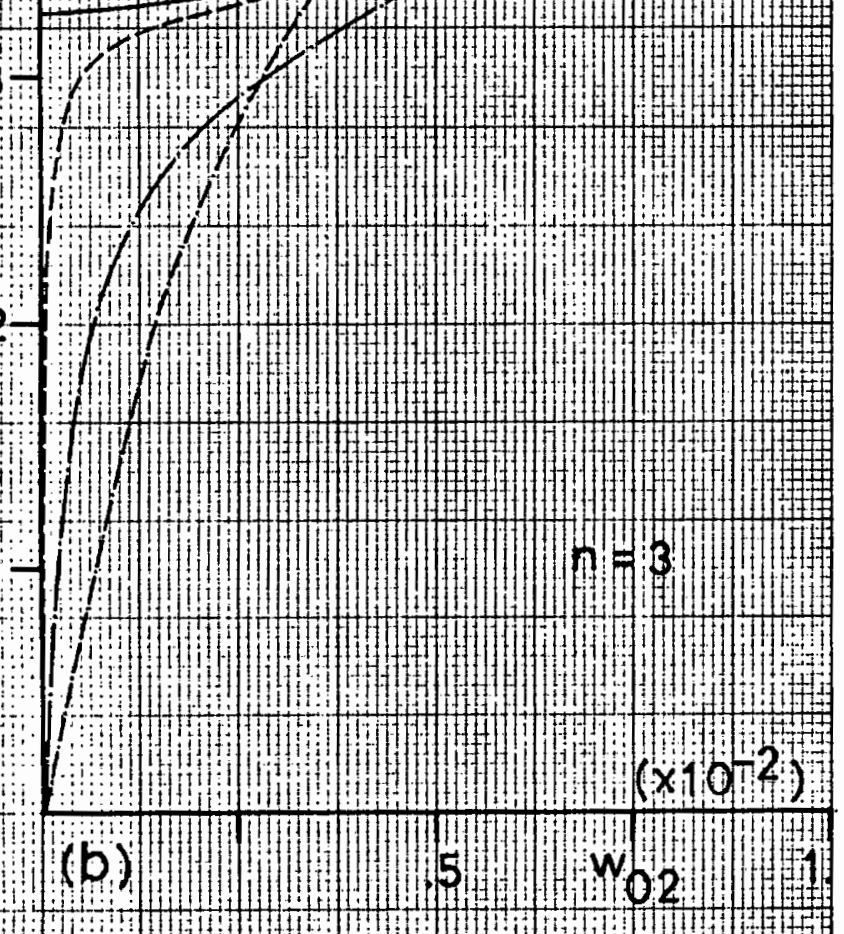

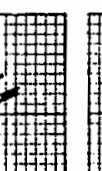




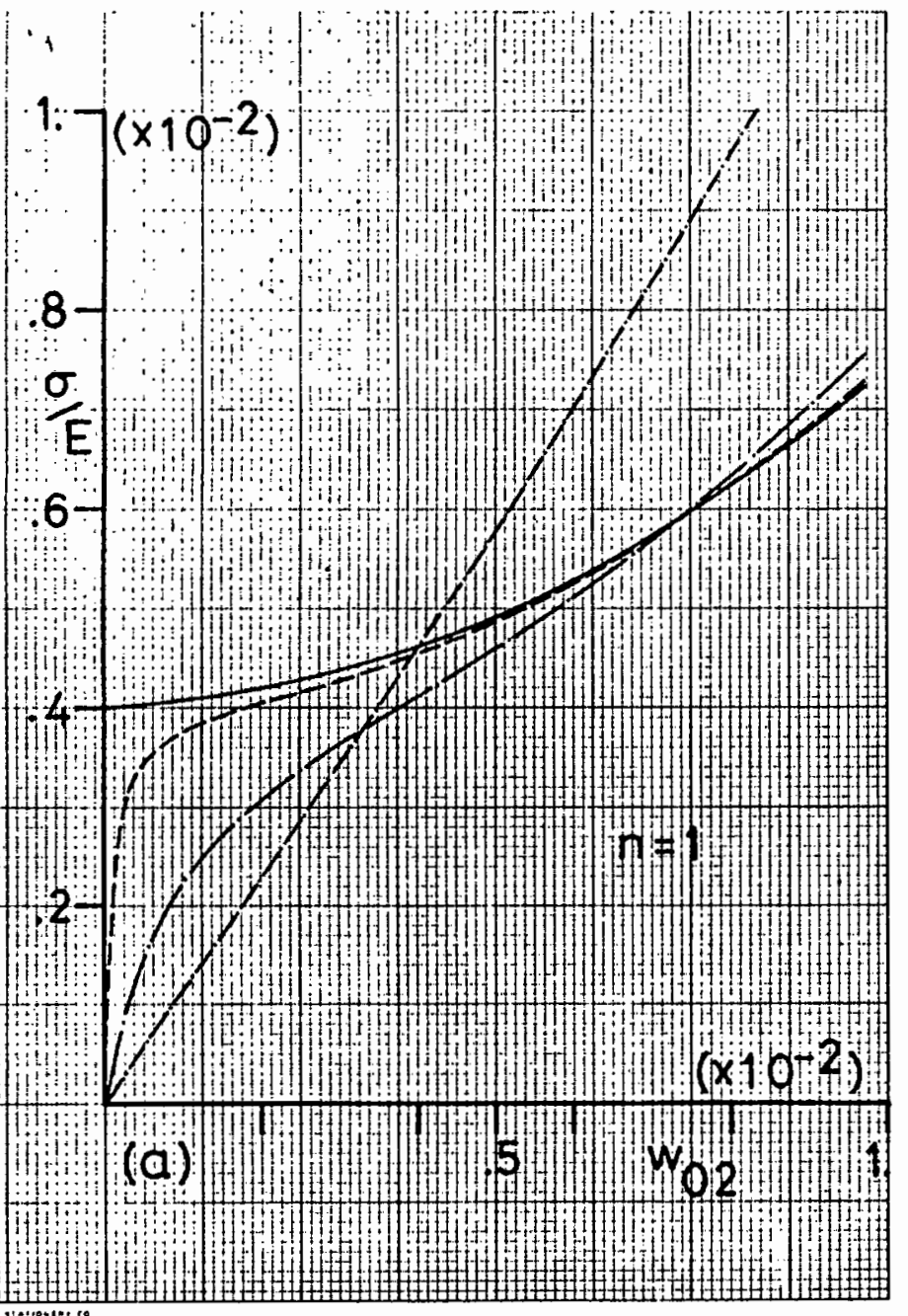

$$
\text { Fig. } \begin{aligned}
& d .8 / t_{s} \\
r & =10 \\
t_{t} & =100
\end{aligned}
$$

\begin{tabular}{|c|c|c|c|}
\hline \multicolumn{2}{|c|}{$\sigma_{c} / E$} & \multicolumn{2}{|c|}{$\left(\times 10^{-2}\right)$} \\
\hline$\mu_{t_{p}}{ }^{n}$ & 1 & 3 & 10 \\
\hline 0 & 0.306 & 0.263 & 0.229 \\
\hline .01 & 0.306 & 0.263 & 0.234 \\
\hline 10 & 0.335 & 0.28 .4 & 0.242 \\
\hline 1.0 & 0.682 & 0.378 & 0.265 \\
\hline
\end{tabular}

Bifurcation stresses
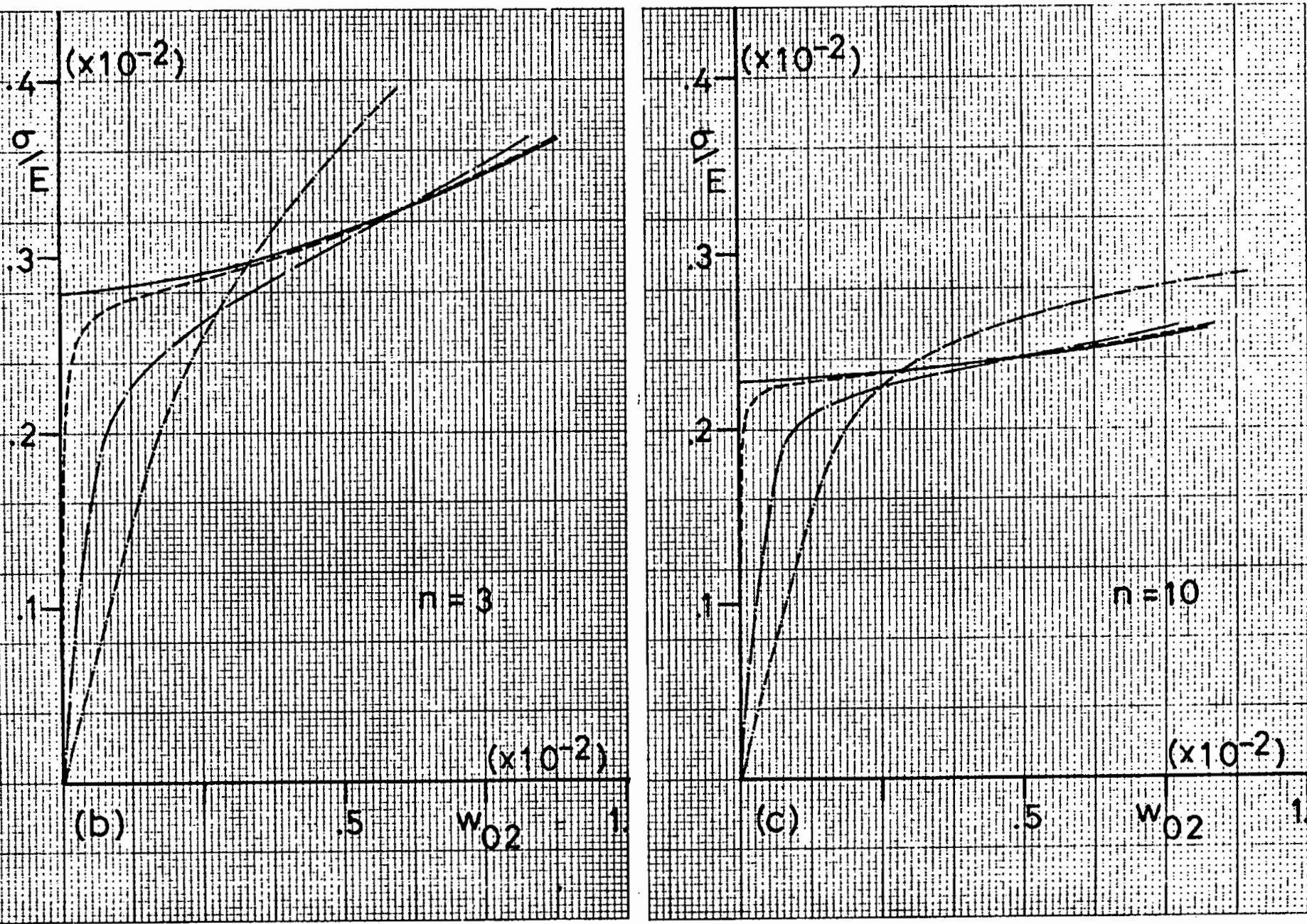


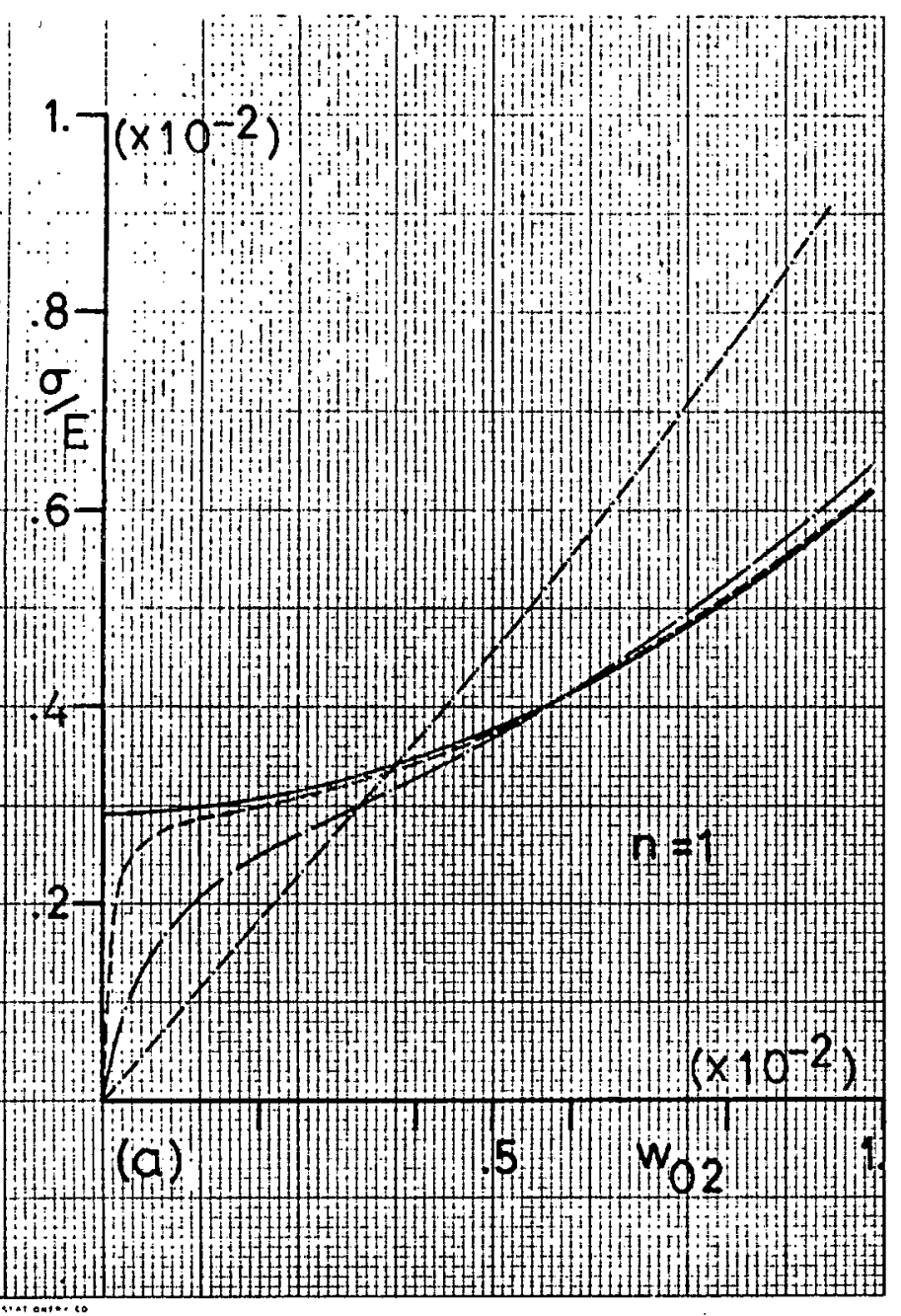

\begin{tabular}{|c|c|c|c|}
\hline \multicolumn{2}{|c|}{ Fig. 7.9} & \multicolumn{2}{|c|}{$d i=10$} \\
\hline & & & 150 \\
\hline \multicolumn{2}{|c|}{$\begin{array}{c}\text { Bifurcation } \\
\sigma_{\mathrm{C}} / \mathrm{E}\end{array}$} & \multicolumn{2}{|c|}{$\begin{array}{l}\text { stresses } \\
\left(\times 10^{-2}\right)\end{array}$} \\
\hline$\mu_{t_{0}}{ }^{n}$ & 1 & 3 & 10 \\
\hline 0 & 0.191 & 0.191 & 0.191 \\
\hline .01 & 0.191 & 0.191 & 0.191 \\
\hline .10 & 0.202 & 0.201 & 0.200 \\
\hline 1.0 & 0.461 & 0.315 & 0.246 \\
\hline$\mu$ & $\begin{array}{l}\text { perfec } \\
t_{p}=0.0 \\
=0.1 \\
=1.0\end{array}$ & & - \\
\hline
\end{tabular}
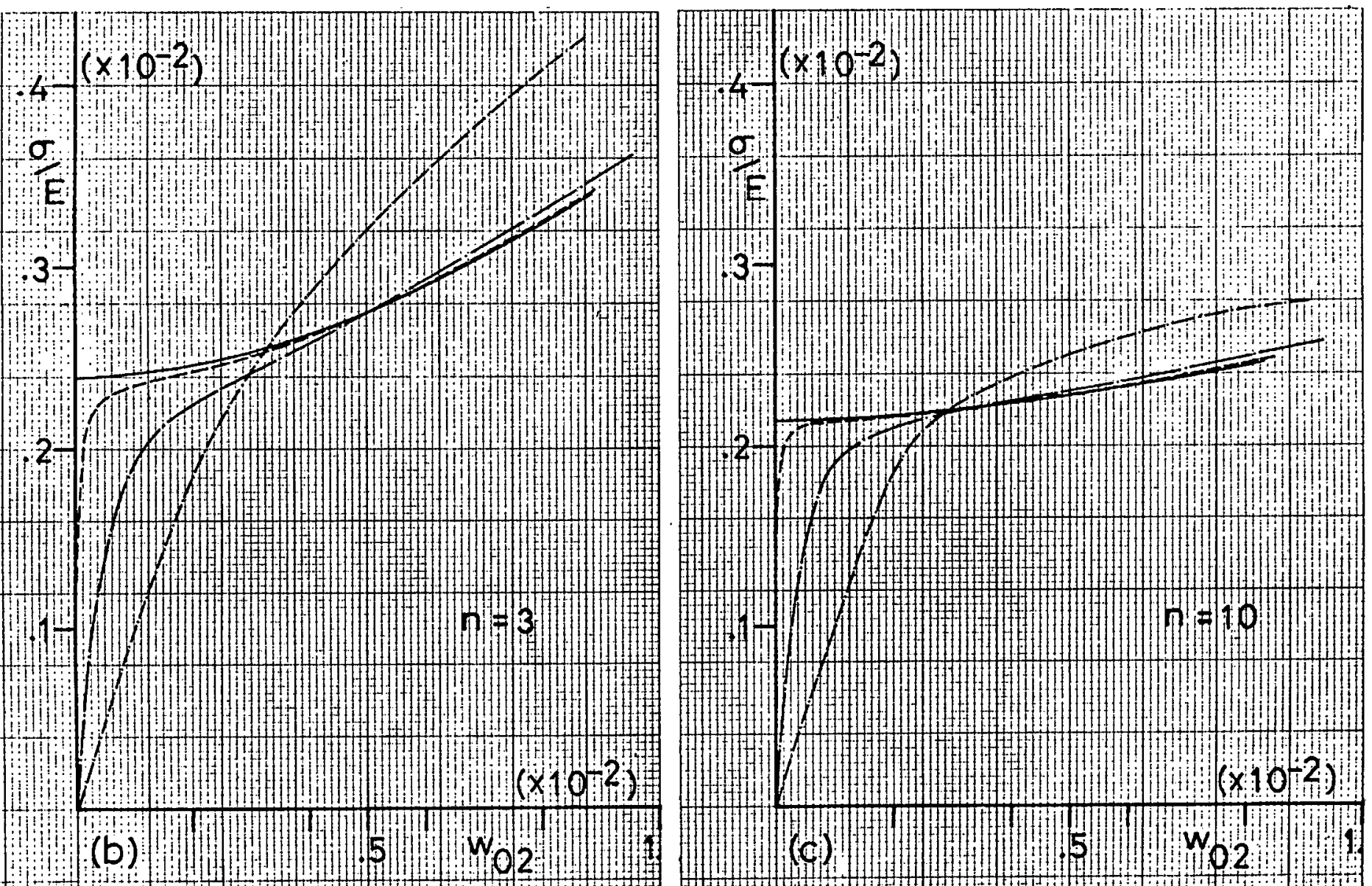


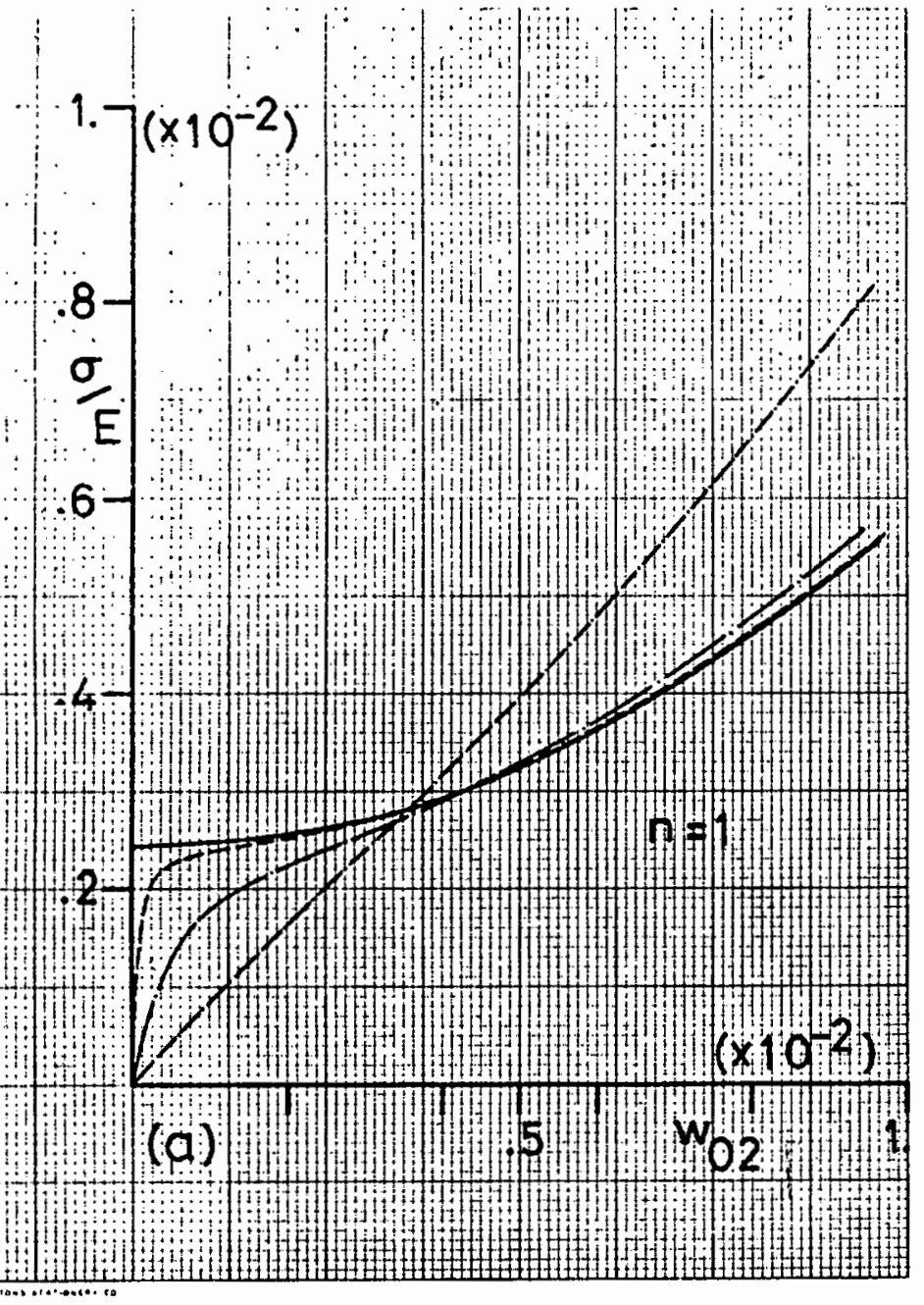

$$
\text { Fig. } \begin{aligned}
7.10 \quad & d_{/ t_{s}}=10 \\
r / t_{p} & =200
\end{aligned}
$$

\begin{tabular}{|c|c|c|c|}
\hline \multicolumn{2}{|c|}{$\sigma_{c} / E$} & \multicolumn{2}{|c|}{$\left(\times 10^{-2}\right)$} \\
\hline$P / t_{p}$ & 1 & 3 & 10 \\
\hline 0 & 0.147 & 0.147 & 0.147 \\
\hline .01 & 0.147 & 0.147 & 0.147 \\
\hline .10 & 0.154 & 0.154 & 0.154 \\
\hline 1.0 & 0.374 & 0.284 & 0.236 \\
\hline
\end{tabular}

Bifurcation stresses
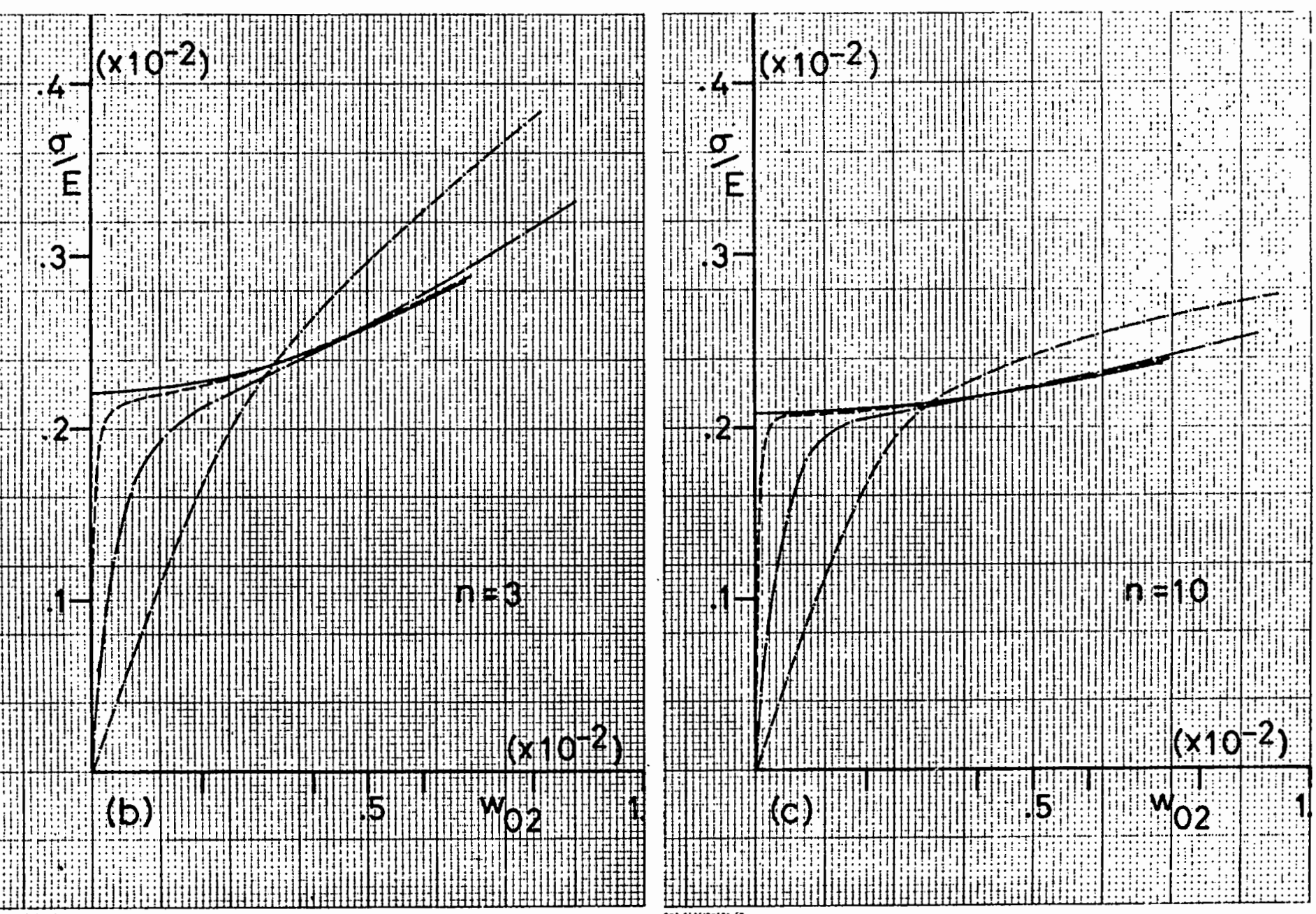
this analysis the non-dimensional imperfection amplitude $\mu / t_{p}=0,0005$ is used to compute the "perfect" post-bifurcation behaviour.

The radial displacement of the panels allows for a "growth" of imperfection (Section 3.7.1) which is determined by the non-dimensional displacement parameter $w_{0_{2}}$. Thus, to be able to observe the effect of the imperfections, curves of external axial stress versus displacement parameter ( $\sigma / E$ versus Wo2 $_{2}$ ) must be obtained. These are shown in Figs. 7.3 to 7.6 for $d / t_{s}=5$ and in Figs. 7.7 to 7.10 for $d / t_{s}=10$; the imperfection mode is that corresponding to $m=2$ and the imperfection amplitudes are $\mu / t_{p}=0,01,0,1$ or $1,0(1 \%$, $10 \%$ or $100 \%$ of panel thickness). All geometric parameters of the shell are kept constant except for those shown in the figures.

The intersection of the "perfect" shell curve and the vertical axis represents the bifurcation point of the perfect shell into the mode that is used for the imperfection in the present analysis. These bifurcation stresses are generally in the same range as those obtained by Reddy (1980) for the same $d / t_{p}$ ratio. Note, however that the shell geometry and the bifurcation mode are different in Reddy's analysis. It is nevertheless helpful to know that these bifurcation stresses are in the correct range. The bifurcation stresses of the perfect shell into a symmetric mode are higher for the shallower stiffener, but note that the differences decrease for lower strain hardening; there is a decrease in critical stress with decreases in strain hardening and panel thickness. These effects agree with Reddy's (1980) results. The postbifurcation paths of the "perfect" shell are predictably stable since the shape of the imperfection corresponds to the most stable bifurcation mode of a perfect cylinder (Syngellakis and walker (1978a)); this mode coincided with the shape of the mid-panel deformation at an advanced stage of loading in the experiment reported in this paper. These post-bifurcation paths are also noticeably flatter for lower strain hardening.

The effects of the imperfection are clearly visible in Figs. 7.3 to 7.10. It is interesting that all the curves for the imperfect shells cross the "perfect" curve at some stage of deformation. Beyond these points it seems beneficial to have imperfections of some magnitude especially since the curves. for higher imperfection values cross the "perfect" curve at lower external stress and lower deformation values. There appears to be some limit to this intersection point because in the lower regions of the curves, before any intersections occur, there is a relatively large difference in behaviour 
between the 18 and $10 \%$ imperfection curves but not such a large difference in behaviour between the $10 \%$ and $100 \%$ curves. This phenomenon is more noticeable for the thicker shells Figs 7.3, 4, 7 and 8. Thus it appears that for large imperfections the differences in behaviour of the shell will be negligible. Generally these intersection points occur after a considerable amount of plastic deformation has taken place.

These intersection points occur at smaller values of $\mathrm{w}_{2}$ for lower strain hardening values. This is due to the degree of plasticity which has caused the post-bifurcation curve of the perfect shell to be flatter for lower strain hardening, which in turn flattens out the other curves as they approach the bifurcation stress of the perfect cylinder. The intersection points also occur at smaller values of wo 2 for thinner panels since the bifurcation stresses are lower than for thicker panels. This is also the reason for the shell with a deeper stiffener having the intersecting points occurring at lower deformations than those of shells with shallower stiffeners.

Within the range of external axial stress and deformations considered the post-bifurcation curve is stable and stiffens while all the imperfect shell curves also start stiffening as the critical stress for the perfect shell is reached. These shell geometries considered have broad panels according to the "flatness" parameter in equation (2.1). This parameter ranges from 1,57 to 2,57 for the shell thicknesses considered. Koiter (1956) determined that a simply supported elastic cylindrical panel in axial compression would have unstable post-buckling behaviour if the "flatness" parameter was larger than 0,64 . Stephens (1971) showed that by taking torsional rigidity of the stiffeners into account, the"flatness" parameter below which stable bifurcation would occur could be significantly increased. This is supported here since by considering the "flatness" parameters of the panels it can be seen that the stiffener must be contributing to the stable behaviour of the shell and thus the stiffening behaviour of the imperfect shells. These results agree with Stephens (1971) and Hutchinson and Amazigo (1967) who have both observed that the effects of the stiffeners could reduce or perhaps eliminate imperfection sensitivity of a shell. It is further interesting that the "perfect" shells with shallower stiffeners have steeper post-bifurcation paths than shells with deep stiffeners only for the elastic case; (compare the (a) diagrams of Figs. 7.3 and $7,7.4$ and $8,7.5$ and $9,7.6$ and 10) and not for the elasticplastic cases (compare the (b) and (c) diagrams of the respective figures). 
The curves for the imperfect shells follow this trend.

\subsubsection{Bifurcation Results}

The bifurcations to be considered in this section are those from the primary path configuration which is symmetric about the mid-generator of the panel into the bifurcation mode which is antisymmetric about the mid-generator. In Figs. 7.3 to 7.10 the critical bifurcation stresses are tabulated for the different shell geometries with differing imperfections and strain hardening parameters. These results are also shown in graphical form in Fig. 7.11 for the shell with $d / t_{s}=5$ and in Fig. 7.12 for the shell with $d / t_{s}=10$. These graphs show plots of critical stress versus the panel thickness parameter $\left(\sigma_{c} / E\right.$ versus $\left.r / t_{p}\right)$. It should be noted that in this section a perfect shell with an imperfection of $0 \%$ is considered.

It can readily be seen by comparing Figs.7.11 and 7.12 that in all instances the shells with the shallower stiffeners bifurcate at higher critical stresses than those with deep stiffeners. The decrease in critical stress with a decrease in panel thickness is clearly shown. This decrease is sharper for the elastic material and flattens out as the strain hardening parameter increases. This is very prominent for the 100\% imperfection. Each individual curve also starts steeply for thick panels and the slope decreases as the panels become thinner. The steep beginning of the elastic curves seems to indicate an upper limit for panel thickness above which bifurcations do not occur. This is not apparent for the $n=3$ and $n=10$ curves but if this does occur then the limit for the panel thicknesses will increase with fand increase in strain hardening parameter. This would mean that a shell with a large panel thickness may be able to bifurcate in the plastic range but will not be able to bifurcate if it remains elastic. Thin shells on the other hand may bifurcate before even reaching a yield point. Thus the curves for different strain hardening parameters should converge to the elastic curve; this is shown for the $0 \%$ and $10 \%$ curves in Fig. 7.12.

The effect of plasticity is to decrease the bifurcation stress and the effect of the imperfections is to the contrary. It can be seen that for the shell with deep stiffeners, large imperfection amplitude and low strain hardening, the effect of the panel thickness is reduced, resulting in the almost horizontal curve on the graph. This is clearly seen for the shell 
Fig. $7.11 \sigma_{c / E}$ vs $r / t_{p}$ for $d / t_{s}=5$.

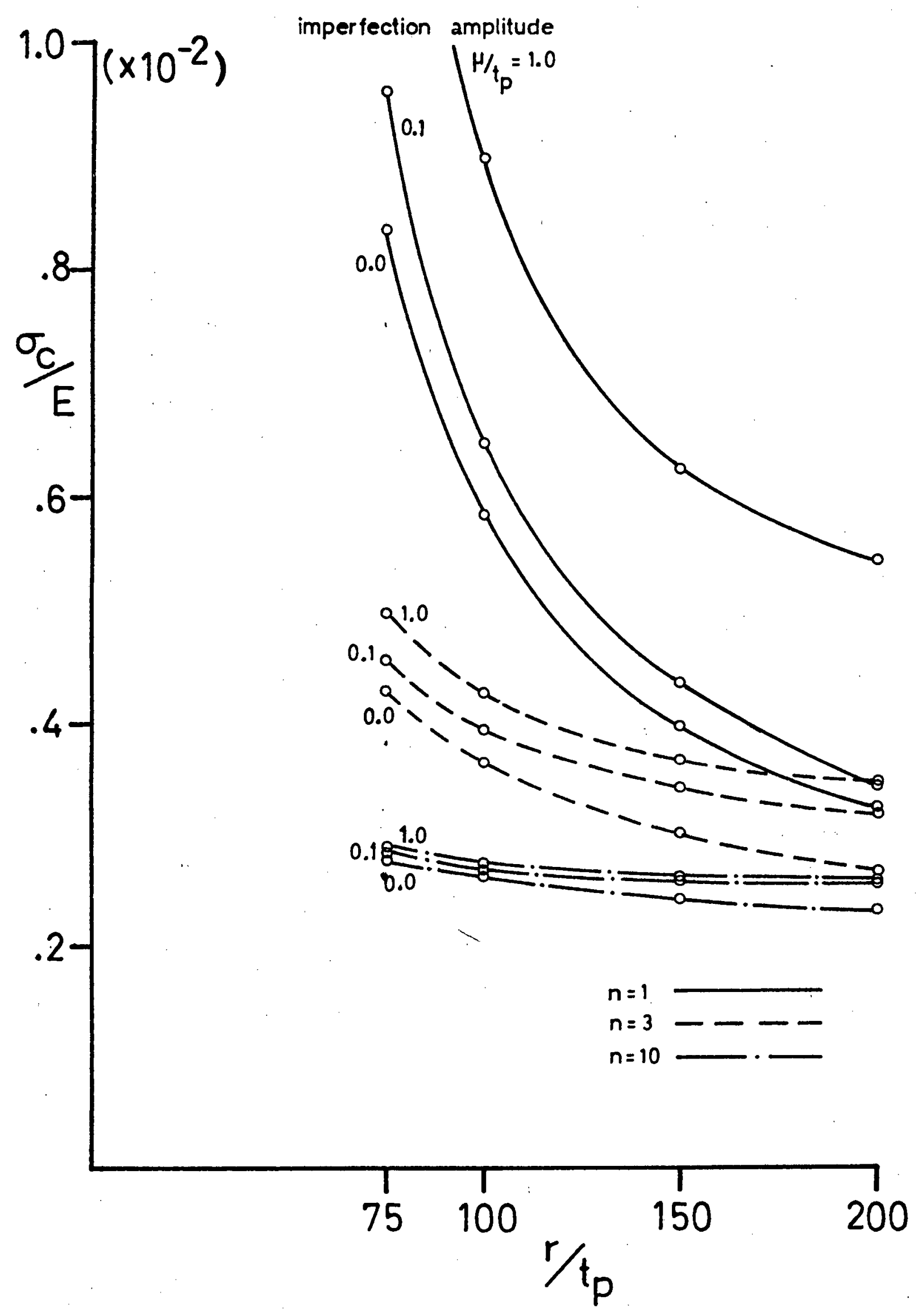


103

Fig. $7.12 \sigma_{c} / E$ vs $r / t_{p}$ for $d / t_{s}=10$.

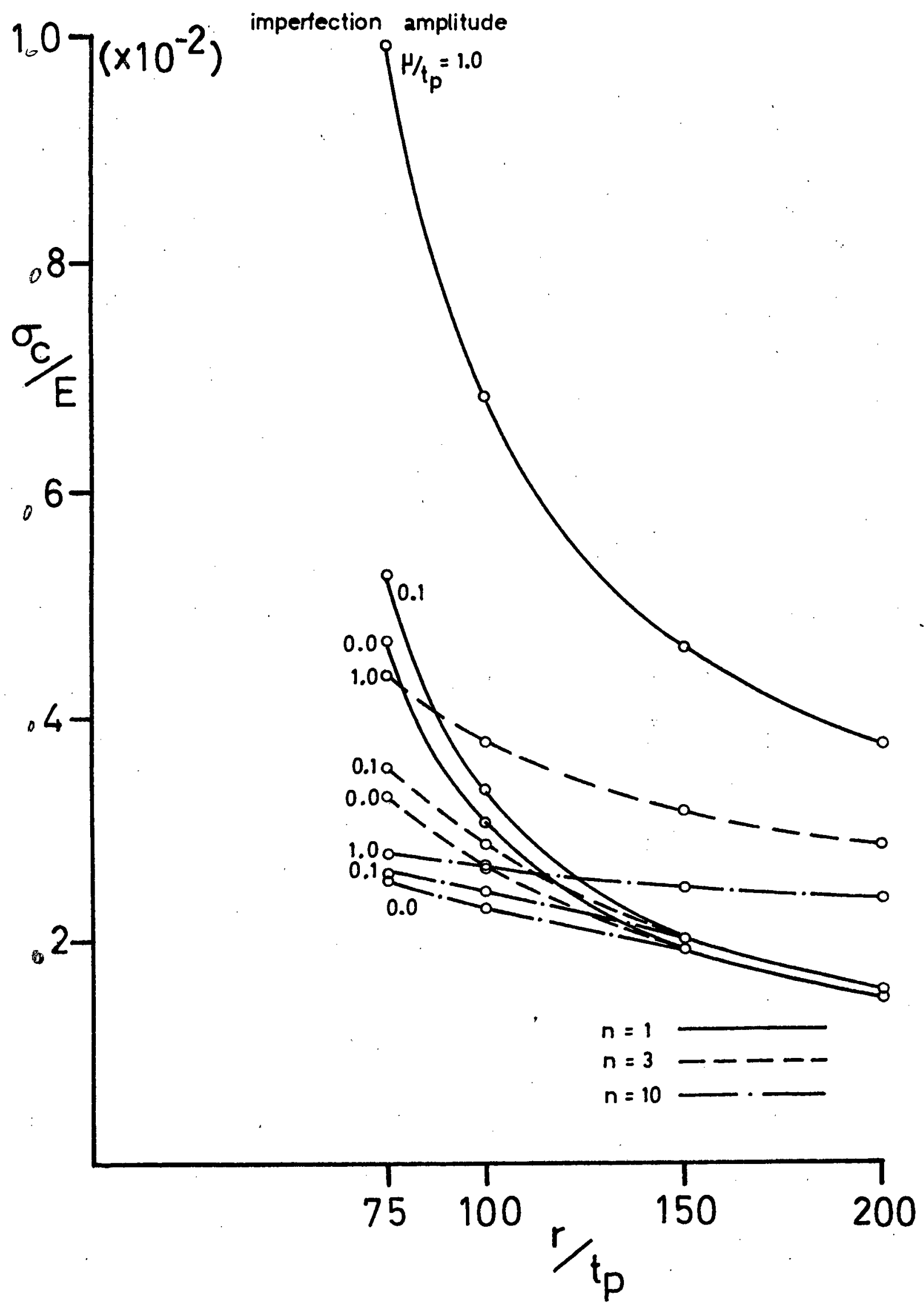


with shallow stiffeners where for $n=10$ and each imperfection amplitude the bifurcation stress is almost the same. Note also the large decrease in critical stress from the elastic curves to the curves for $n=3$, but a smaller decrease in critical stress from the $n=3$ to the $n=10$ curves.

\subsection{Comparisons with Experimental Results}

The experimental curve as given by Syngellakis and Walker (1978b) from their experiment is shown in Fig. 7.1. This curve was obtained for a simply supported araldite shell. The theoretical elastic critical stress compares favourably with the experimentally found deviation from the normal loading curve. By introducing a yield value and strain hardening exponent into the analysis the critical stress could be lowered for better agreement but this would be meaningless as the behaviour of araldite beyond the elastic limit is unknown.

Numerical analyses were carried out on the shells tested by Walker and Davies (1976). There are three different shell geometries and the resulting curves are shown together with the approximate experimental curves of walker and Davies (1976) in Figs. 7.13, 14 and 15. These theoretical curves are plotted for a strain hardening parameter $n=12$, with $m=6$ and for the imperfections given by the out of roundness parameters in the paper. A theoretically obtained curve for a large imperfection is also shown in Fig. 7.13. The straight lines in the graphs are the elastic loading curves as given in the paper.

It is quite clear that the theoretical curves are much steeper and do not compare favourably with the experimental results. There are several possible reasons for this: firstly, the experimental results are obtained for stiffened shells that have their ends rigidly damped. Since the test specimens were relatively short $(\ell / \mathrm{r} \simeq 1,5)$ one would expect the end conditions to have a significant effect on the behaviour of the shell.

Secondly, the B1 shell (Fig. 7.14) has a "flatness" parameter of 0,76 which indicates the narrow panels and therefore a possibility of an overall mode of buckling which includes the stiffeners in the radial deformations of the buckled mode; this overall mode is shown in the paper combined with a local mode. This combination of modes could have caused failure of the shell at a lower stress than predicted for either mode. The theoretical curve for 
Fig. 7.13 Curves for A2 cylinder.

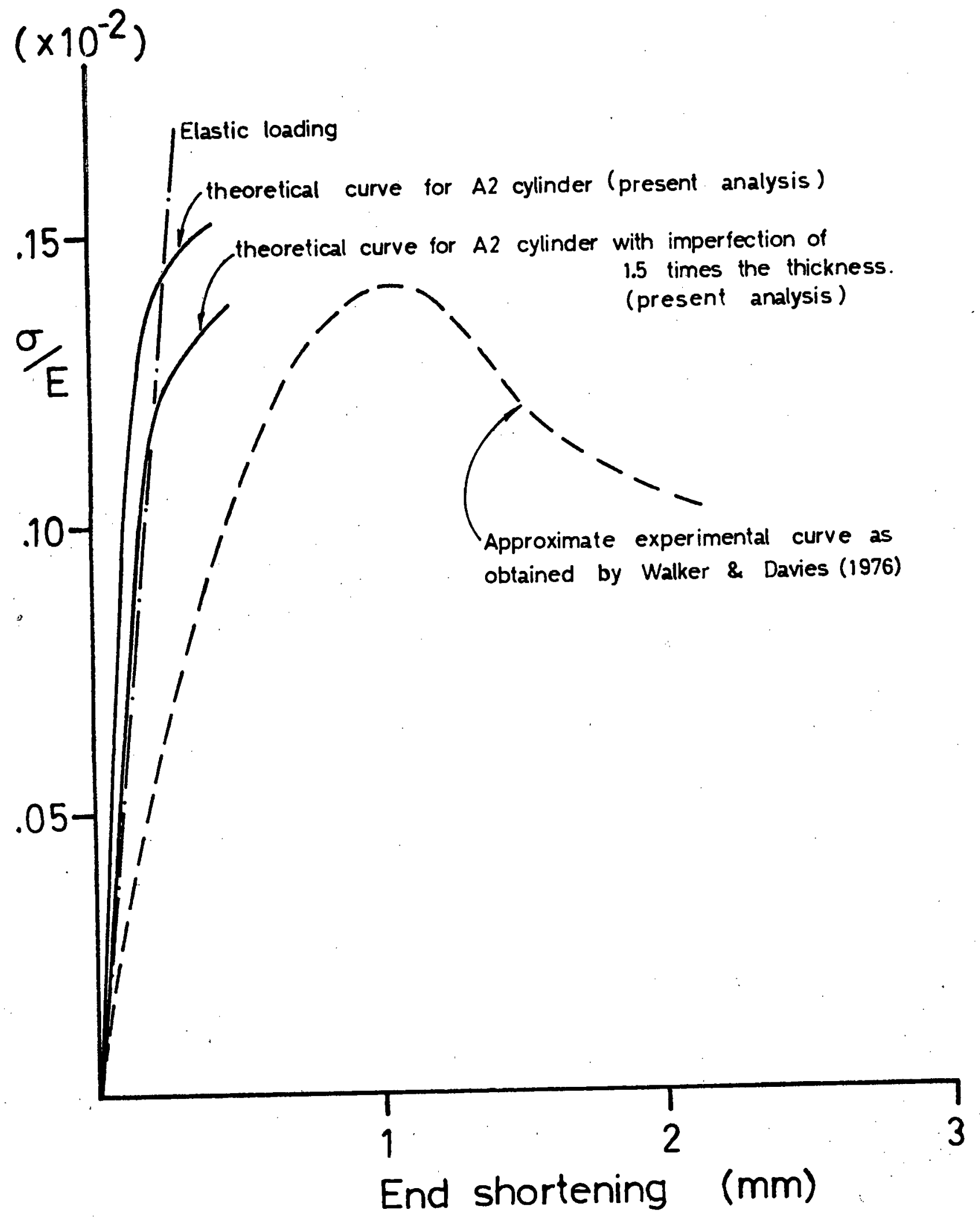


Fig. 7.14 Curves for B1 cylinder.

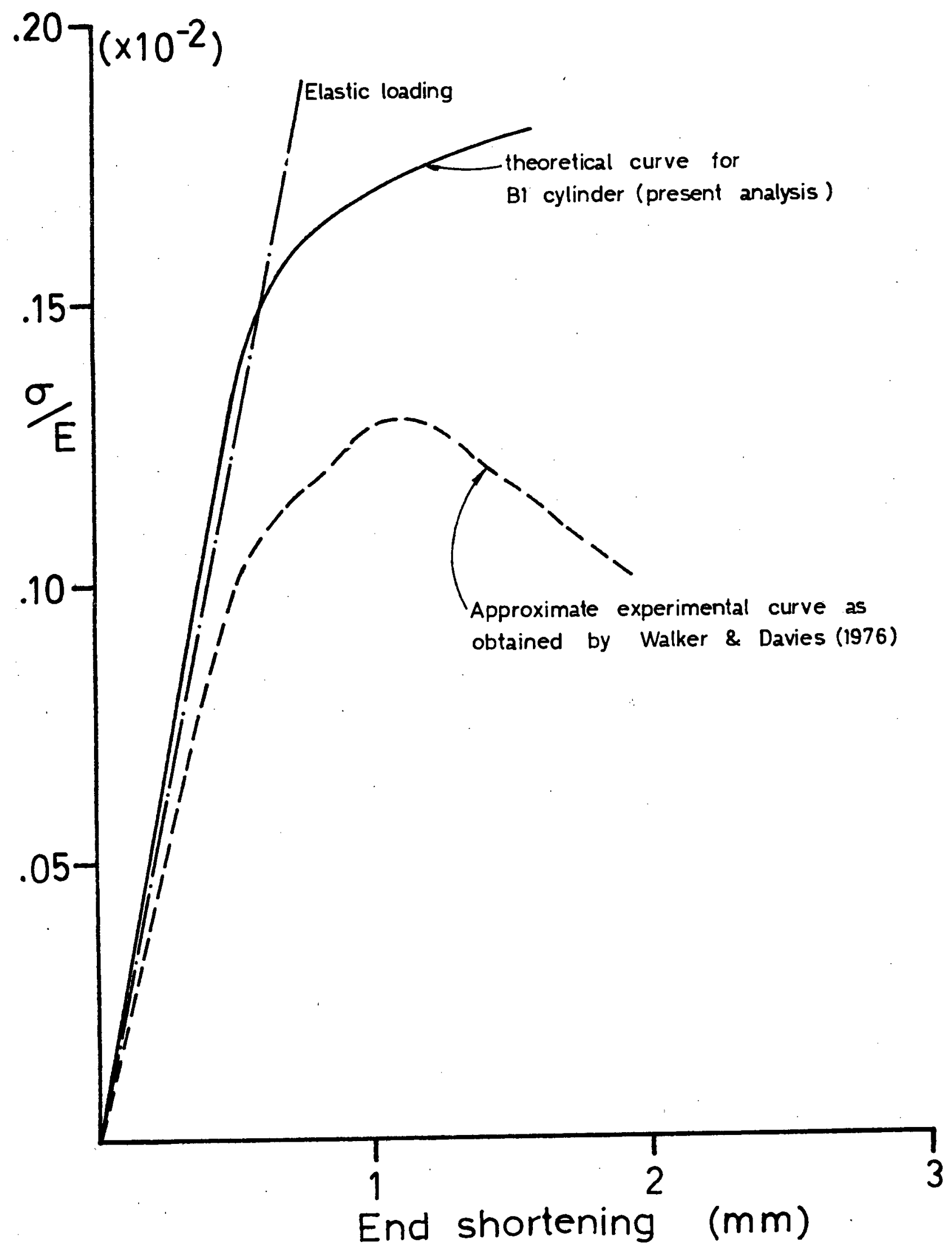


Fig. 7.15 Curves for B2 cylinder.

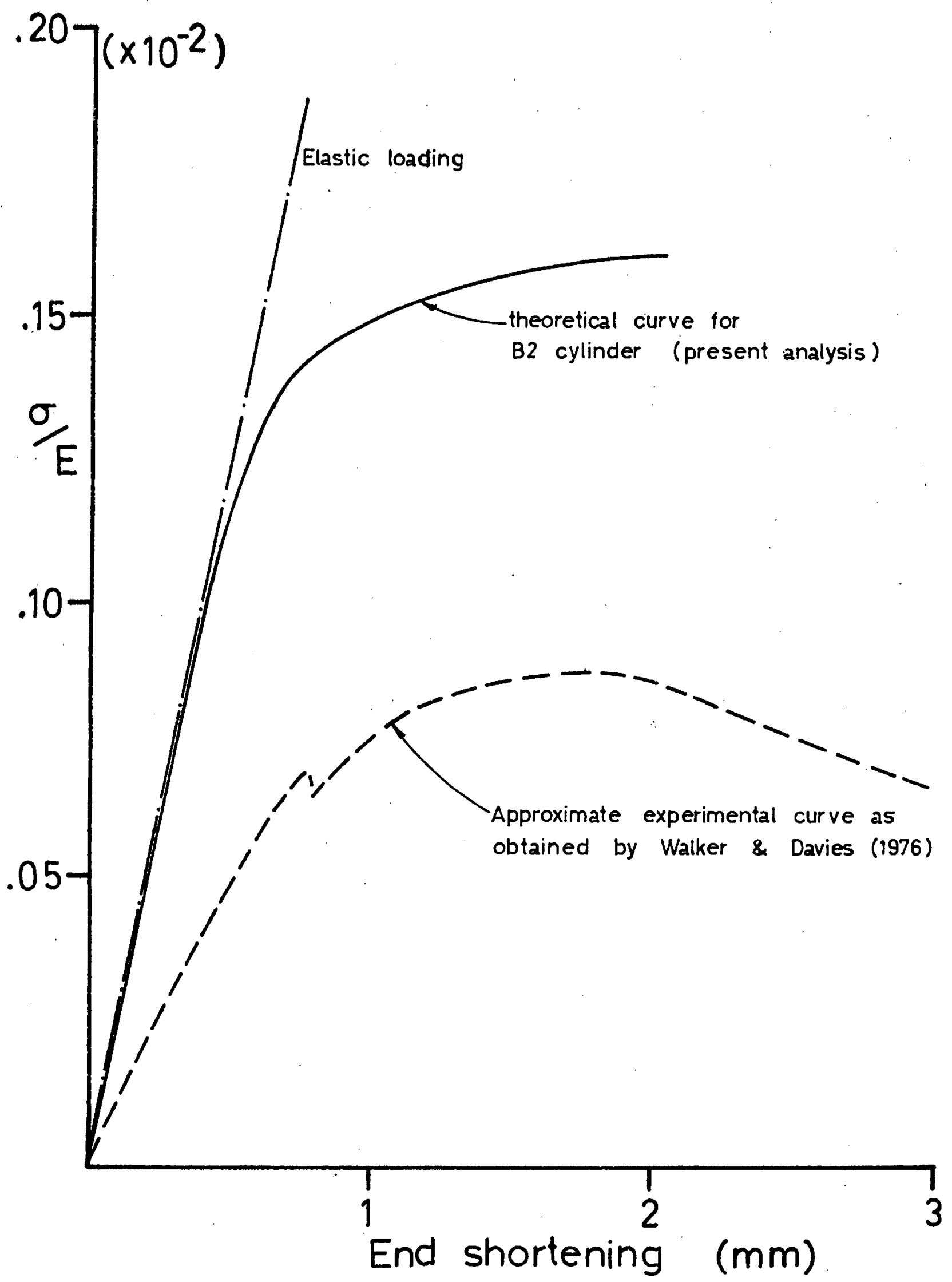


the sparsely-stiffened A2 shell (Fig. 7.13) begins to flatten out at a slightly higher stress than the experimental curve; however, prior to this the theoretical curve is much steeper than the experimental curve. The second theoretical curve for an imperfection of 1,5 times the shell thickness has a reduced slope and also flattens out at a lower load than the previous curve but it is still much different from the experimental curve. For the B2 shell as shown in Fig. 7.15 there is little comparison between the theoretical and experimental curves. It must be noted that no bifurcation points were located up to the external stresses at which these analyses were terminated.

The welding of the stiffeners and the panels to form the cylinders, in spite of precautions, creates high temperatures at the panel-stiffener junction which must result in residual stresses in the shell at these critical positions. These residual stresses could have a significant effect on the behaviour of the shell. It should be noted that the experimental curves deviate from the lines of elastic loading from the very beginning of the experiments. This could be due to the combined effects of the end support conditions and the residual stresses.

It should also be mentioned that the imperfection parameters given in the paper by Walker and Davies (1976) may be lower than the actual imperfections due to the difficulty in obtaining these parameters. It is seen in Fig. 7.13 that the curve for a cylinder with a larger imperfection has a less steep slope and begins to flatten out earlier. It appears that this is not a real source for the discrepancies since, judging from Fig. 7.13, the two theoretical curves are not vastly different compared to the differences in imperfection amplitude. It should also be noted that the imperfections given in the paper do not have the same shape as those assumed in this analysis.

\section{5 Concluding Remarks}

The effects of plasticity and of initial imperfections have been shown in this chapter for a few particular geometries of the stiffened cylinder. The changes in bifurcation stress with respect to the thickness of the panels has been disassed. It is unfortunate that a complete analysis is almost an impossible task and that many aspects cannot be covered here. Comparison with experimental data for a simply supported shell was favourable, but comparison with the fixed ended shell experiments of Walker and Davies

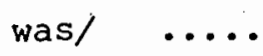


was not very successful. The effects of different boundary conditions and of residual stresses, which may be the cause of the discrepancies discussed, are not included in this present analysis. 


\section{CHAPTER 8}

\section{CONCLUSIONS}

An analysis of the behaviour and bifurcation characteristics of a stiffened cylindrical shell with an initial stress-free imperfection has been presented. The shell was simply supported at its ends and subject to an axial compressive load. The material model used was $\mathrm{J}_{2}$ deformation theory.

The effect of the imperfections on the primary equilibrium path have been investigated, and results have been presented showing the load-deformation behaviour of shells with different panel thicknesses subject to various imperfections. The investigation has revealed that the behaviour of an imperfect shell tends towards the post-bifurcation path of the perfect shell but then stiffens as the external axial stress approaches the critical stress of the perfect cylinder, causing the imperfect shell path to intersect with that of the post-bifurcation path of the perfect shell. Below this intersection point imperfect stiffened cylinders have larger deformations than the perfect shell for a given load, the amount of deformation increasing with the imperfection, but beyond these points the cylinders with the large imperfections have smaller deformations than those with smaller imperfections. It was noticed that the larger the imperfection amplitude the lower the external stress and deformation at which this intersection occurs; however, there appears to be some limiting value for each particular case. It must be noted that thése intersection points generally occur after a considerable amount of plastic deformation.

This lack of imperfection sensitivity can be attributed to the torsional effects of the stiffeners which, for the examples considered, have significantly reduced the imperfection sensitivity. This agrees with results found by Hutchinson and Amazigo (1967) and Stephens (1971) that the effects of stiffeners can significantly reduce or perhaps even eliminate imperfection sensitivity. Further investigation into the effects of different stiffener sizes on shells of this type are required to evaluate their exact effect on the imperfection sensitivity of the shell.

Secondly, the effects of the imperfections on the critical bifurcation stresses for elastic and plastic materials have been studied. At these critical stresses the cylinder bifurcates into a mode which is anti- 
symmetric with respect to the panel mid-generators. From this bifurcation analysis it is evident that shells with shallow stiffeners yield higher critical stresses than shells with deeper stiffeners, whether the material be elastic or plastic, although as the strain hardening is reduced this difference diminishes. It is also clear that the critical stress is reduced for shells having thin panels. This reduction in critical stress as the panels get thinner is more pronounced for shells with high strain hardening and can be almost negligible for low strain hardening materials. There appears to be some limit on the panel thicknesses above which bifurcations of this type cannot occur for a particular strain hardening parameter. At the other extreme thin shells may buckle in the elastic range before they even reach a yield point. Generally the onset of plasticity tends to decrease the critical stresses while the effect of the initial imperfections is to increase the critical stress. This increase due to the imperfections is more prominent in the high strain hardening range.

An attempt has been made to compare theoretical results with particular experimental results. For stiffened cylinders that are simply supported at their ends a good comparison has been achieved with the experimental result of syngellakis and walker (1978b) although this comparison has had to be confined to the elastic range. The experimental results obtained by Walker and Davies (1976) for steel cylinders were compared with the theoretical results from the present analysis. The theoretical load-deformation curves do not correspond at all well with the experimentally obtained curves. A number of possible reasons for this discrepancy have been advanced: firstly, the experimental curves are obtained for cylinders that have clamped ends and since the shells are relatively short the end conditions are expected to have a significant effect. Secondly, these experimental curves deviate from elastic loading from the very beginning of the experiment and it is thus suspected that the high temperatures caused by welding of the stiffeners and panels in manufacture have created residual stresses in the critical areas around the panel-stiffener junctions which have altered the load-deformation behaviour. These two effects are not included in the present analysis. The inclusion of these two effects into the analysis provides much scope for future work in this field. 


\section{REFERENCES}

BATHE, K.J. and CIMENTO, A.P. (1980). Some practical procedures for the solution of nonlinear finite element equations, Computer methods in Applied Mechanics and Engineering, 22, 59-85.

BUDIANSKY, B. (1959). A reassessment of deformation theories of plasticity, J. Appl. Mech., 26, 259-264.

CRISFIELD, M.A. (1980). The automatic nonlinear analysis of stiffened plates and shallow shells using finite elements, Proc. Instn.Civ. Engnrs., Part 2, 69, Dec., 891-909.

DAHLQUIST, G. and BJORCK, A. (1974). "Numerical Methods", Prentice-Hall, Inc., Englewood Cliffs, New Jersey.

GELLIN, S. (1979). Effect of an axisymmetric imperfection on the plastic buckling of an axially compressed cylindrical shell, J. Appl. Mech., 46, 125-131.

HUTCHINSON, J.W. (1974). Plastic Buckling, Advances in Applied Mechanics, 14, 67-144.

HUTCHINSON, J.W. and AMAZIGO, J.C. (1967). Imperfection-sensitivity of eccentrically stiffened cylindrical shells, AIAA Journal, Vol.5, No. 3, 392-401.

JENNINGS, A. (1971). Accelerating the convergence of matrix iterative processes, J. Inst. Maths Applics, 8, 99-110.

KOITER, W.T. (1956). Buckling and post-buckling behaviour of a cylindrical panel under axial compression, National Aeronautical Research Institute; Amsterdam, Report S.476.

MALVERN, L.E. (1969). "Introduction to the mechanics of a continuous medium" , Prentice-Hall, Inc., Englewood Cliffs, New Jersey. 
NEEDELMAN, A. (1975). Post-bifurcation behaviour and imperfection sensitivity of elastic-plastic circular plates, Int. J. Mech. Sci., Vol. 17, No. 1, 1-13.

REDDY, B.D. (1980) . Buckling of elastic-plastic discretely stiffened cylinders in axial compression, Int. J. Solids Structures, 16, 313-328.

SANDERS, J.L. (1963). Nonlinear theories for thin shells, Quarterly of Appl. Math., 21, 21-36.

STEPHENS, W.B. (1971) . Imperfection-sensitivity of axially compressed stringer reinforced cylindrical panels under internal pressure, AIAA Journal, Vol. 9, No. 9, 1713-1719.

STROUD, A.H. and SECREST, D. (1966). "Gaussian quadrature formulas", Prentice-Hall, Inc., Englewood Cliffs, New Jersey.

SYNGELLAKIS, S. and WALKER, A.C. (1978a). Elastic buckling of stiffened cylindrical shells, Int. Symp. on Integrity of Off-shore structures, Glasgow.

SYNGELLAKIS, S. and WALKER, A.C. (1978b). Elastic local buckling of longitudinally stiffened cylinders, Conf. on Stability Problems in Engineering Structures and Components, Cardiff.

TVERGAARD, V. (1977). Buckling of elastic-plastic cylindrical panel under axial compression, Int. J. Solids Structures, 13, 957-970.

WALKER, A.C. and DAVIES, P. (1976). The collapse of stiffened cylinders, Int. Conf. Steel Plated Structures, Imperial College, London.

WALKER, A.C. and SRIDHARAN, S. (1980). Analysis of the behaviour of axially compressed stringer-stiffened cylindrical shells, Proc. Instn. Civ. Engrs., Part 2, 69, June, 447-472.

WANG, J. T-S. and LIN, Y-J. (1973) . Stability of discretely stringerstiffened cylindrical shells AIAA Journal, Vol. 11, No. 6, 810-814. 